\title{
The influence of nutrient availability on the thermotolerance of symbiotic dinoflagellates of family \\ Symbiodiniaceae
}

\author{
Evan Raymond \\ A thesis submitted to the Victoria University of Wellington \\ in partial fulfilment of the requirements for the degree of \\ Master of Science \\ in Marine Biology
}

Victoria University of Wellington, New Zealand

2021 


\section{Acknowledgements}

I would like to thank all those who contributed their valuable time and energy in support of my research aspirations. I would first like to thank my supervisor, Professor Simon Davy, not only for his critical support and feedback throughout my research and thesis writing, but also for recognising my potential to pursue post-graduate study in the first place.

I would also like to acknowledge the entire Davy lab group for their advice and guidance over the past two years. In particular, thanks to Dr. Clint Oakley for keeping me well supplied with antibodies and various other miscellaneous and/or obscure reagents, and also to (soon to be Dr.) Amirhossein Mashini for his patience in answering my incessant questions about western blot protocol.

A special thank you goes to Patricia Stein for all her help during my time at VUW and without whom I would have probably starved to death long ago.

Most of all I would like to thank my parents for their constant support from day one, and for instilling in me the confidence to always pursue my goals, no matter how unorthodox or temporally inexplicable they may seem. 


\begin{abstract}
Coral reefs are the most biodiverse ocean ecosystems on the planet, providing essential habitat for over $25 \%$ of the world's marine organisms. Their structural complexity and stability contribute to essential coastline defence against erosion, as well as provide billions of dollars per year in economic value in the form of tourism and artisanal fishing. Fundamental to this unique and indispensable habitat is the symbiotic relationship between cnidarian corals and algal dinoflagellates of family Symbiodiniaceae. Reef-building corals gain a crucial majority of their daily energy needs from their endosymbiotic dinoflagellates, which facilitates coral growth, reproduction and formation of the reef structure on which countless other organisms thrive. However, this symbiosis has come under threat from warming oceans as a consequence of anthropogenic climate change. Under thermal stress the cnidariandinoflagellate symbiosis breaks down, resulting in expulsion of the dinoflagellate ('coral bleaching'), followed by the eventual death of the coral animal. Tolerance of elevated temperatures is known to vary among coral and Symbiodiniaceae species, and may be influenced by the interaction of nutrient availability and photosynthetic function of the endosymbiont.

The aim of this thesis was to investigate photophysiological mechanisms in thermotolerant and thermally sensitive Symbiodiniaceae to determine how they are affected by thermal and nutritive stress, both in and out of symbiosis.
\end{abstract}

Cultured Symbiodiniaceae phylotypes A4 (thermotolerant) and B2 (thermally sensitive) were subjected to high and low nutrient and temperature treatments. The photosynthetic health (quantum yield, $\mathrm{F}_{\mathrm{V}} / \mathrm{F}_{\mathrm{M}}$ ), rate of chloroplastic protein synthesis (D1 protein) and photophysiological response to light (NPQ) of each phylotype was monitored to determine if thermotolerance was related to nutrient utilization under heat stress. Phylotype A4 showed considerably increased D1 synthesis regardless of nutrient treatment when compared to phylotype $\mathrm{B} 2$, but only minor differences in $\mathrm{F}_{\mathrm{V}} / \mathrm{F}_{\mathrm{M}}$. Also, correlation between $\mathrm{D} 1$ concentration and $\mathrm{F}_{\mathrm{V}} / \mathrm{F}_{\mathrm{M}}$ was observed in $\mathrm{A} 4$, but not $\mathrm{B} 2$ during recovery from heat stress. Responses 
to short term light exposure contrasted significantly between the two phylotypes under all conditions, indicating marked differences in the photophysiological apparatus.

To examine nutrient use and thermotolerance in symbiosis, the model symbiotic anemone Aiptasia pallida (commonly Aiptasia) was inoculated with either Symbiodiniaceae species Breviolum minutum, phylotype A4 or phylotype B2. The holobionts (host and symbiont) were fed or starved for a period of six weeks and then subjected to heat stress. D1protein concentration and $\mathrm{F}_{\mathrm{V}} / \mathrm{F}_{\mathrm{M}}$ was similar in all fed holobionts, regardless of symbiont type and heat treatment. Following heat stress, all starved holobionts showed extremely low concentrations of $\mathrm{D} 1$ protein, but comparable $\mathrm{F}_{\mathrm{V}} / \mathrm{F}_{\mathrm{M}}$, while in low temperature starved treatments, only Aiptasia hosting B. minutum showed any recovery of D1 protein.

The study shows that efficiency of nutrient utilization in photosynthetic pathways is not necessarily an indicator of thermotolerance, nor does it dictate the ability of the symbiont to confer physiological benefits to the host under conditions of heat or nutrient stress. Rather, host-symbiont pairings most likely reflect responses to external pressures dictated by the local environment. The implications of the physiological disparities between the Symbiodiniaceae types tested are discussed in the context of environmental adaptations and host-symbiont nutrient dynamics. The complexity of symbiotic interactions highlighted by this study reinforces the imperative necessity of further investigations into cnidarian-dinoflagellate symbioses, particularly in regard to thermotolerance and photophysiology. Only through understanding the physiological effects of rising ocean temperatures on this essential partnership can we begin the work of protecting the coral reef habitat for future generations. 


\section{Table of Contents}

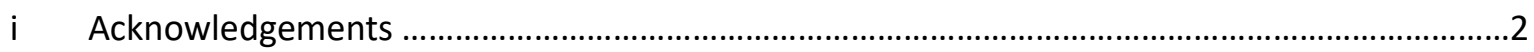

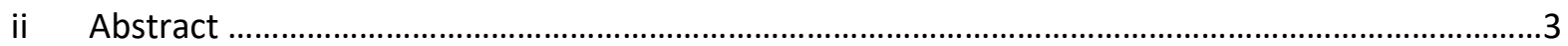

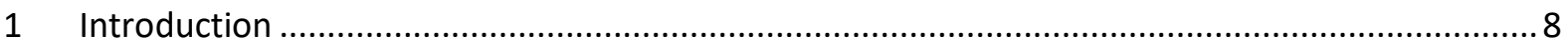

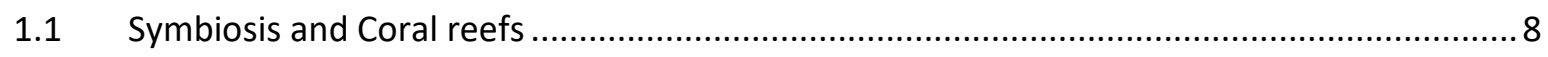

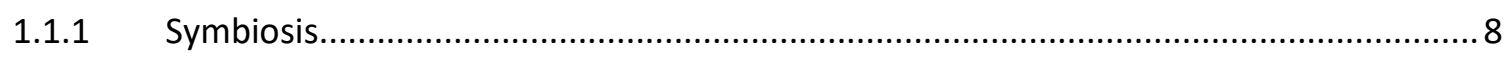

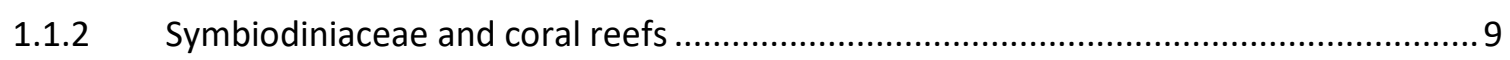

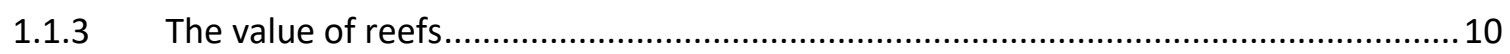

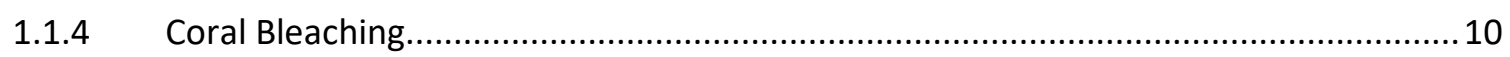

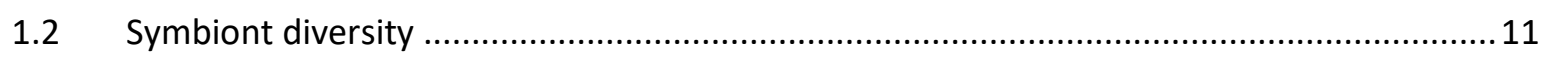

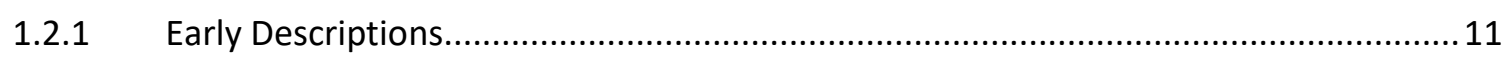

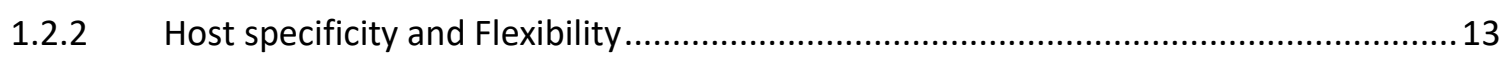

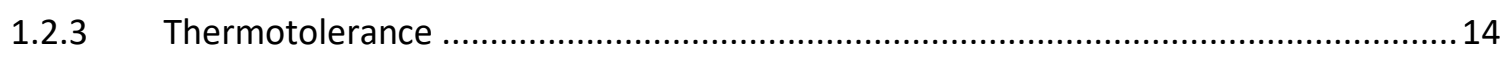

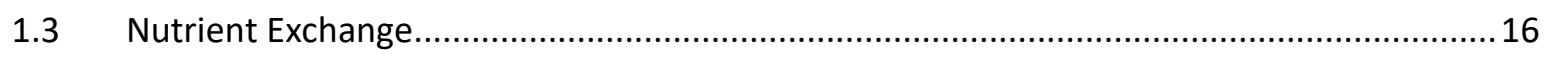

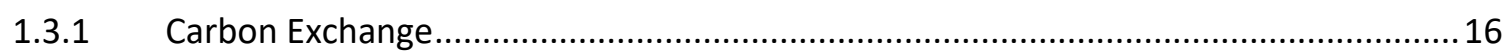

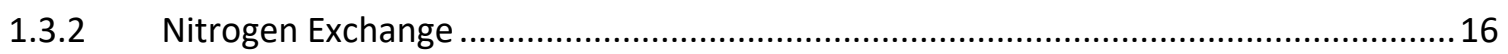

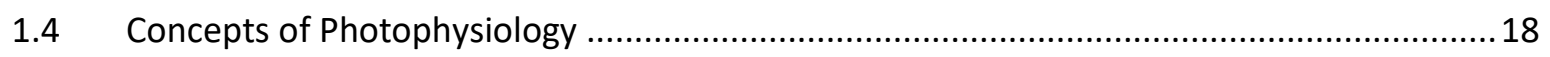

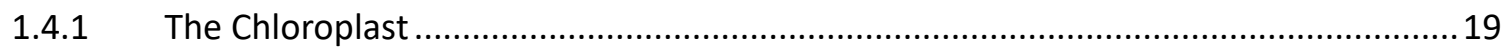

1.4.2 The Photosystems and the electron transport chain ................................................ 19

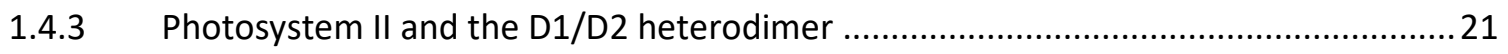

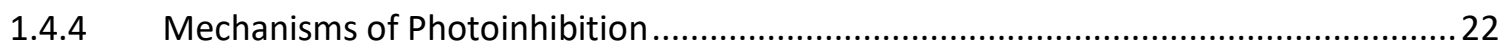

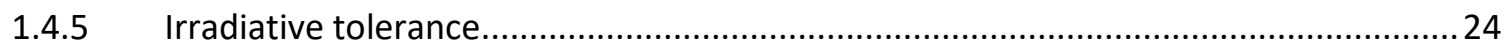

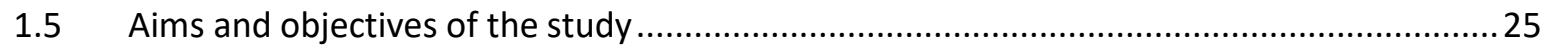

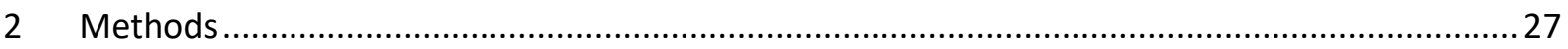

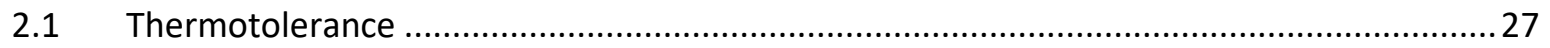

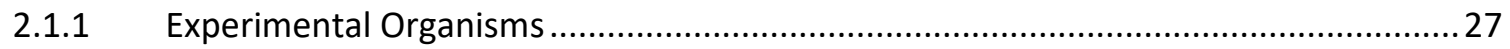

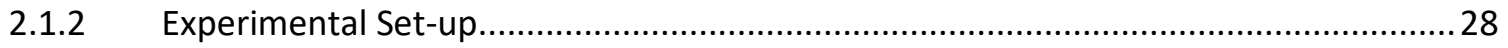

2.1.3 Photosynthetic performance and thermotolerance ......................................................29

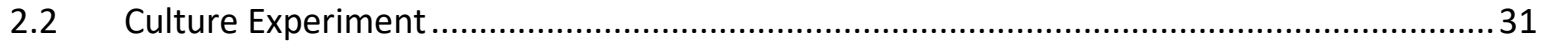

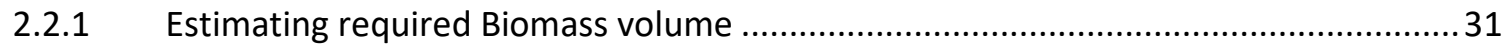

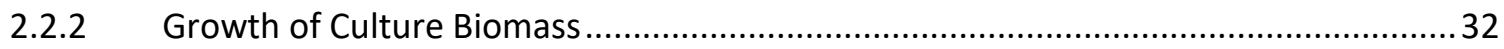

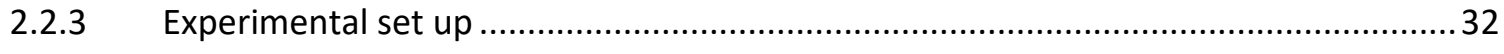

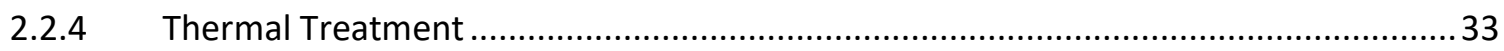




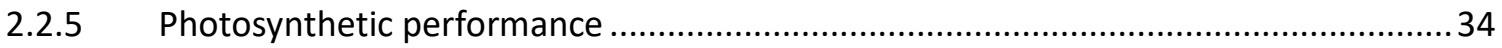

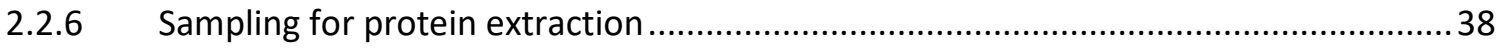

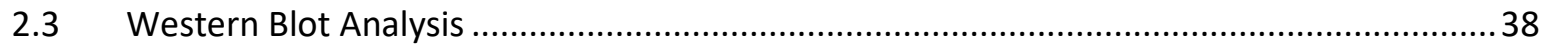

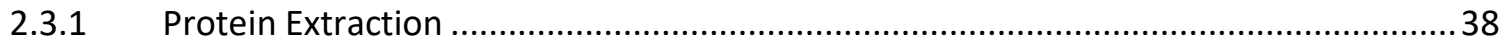

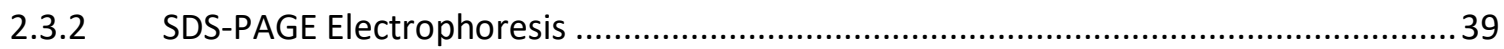

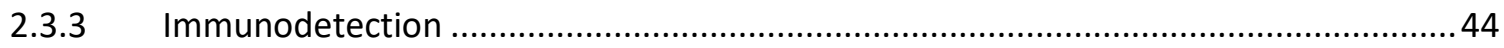

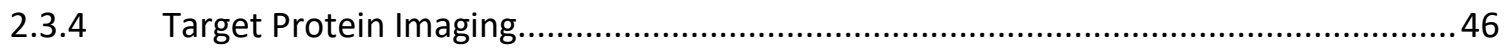

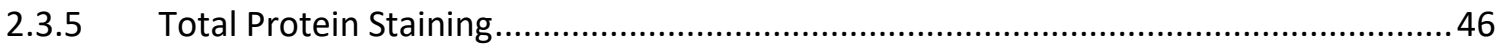

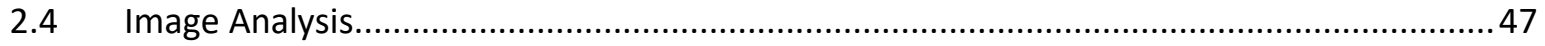

2.4.1 Target Protein Optical Densitometry ....................................................................... 47

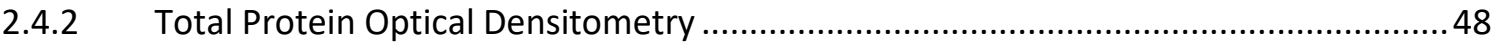

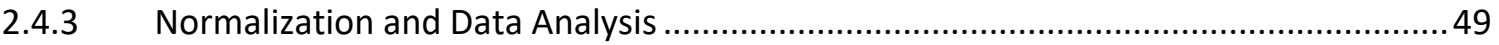

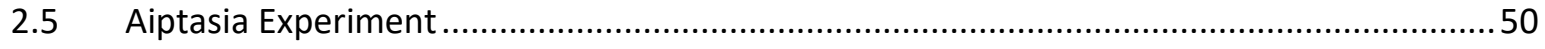

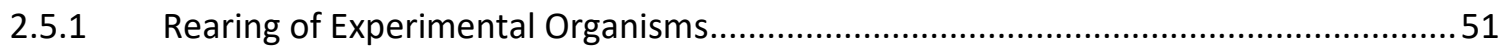

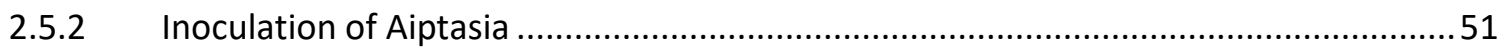

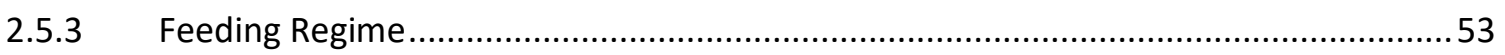

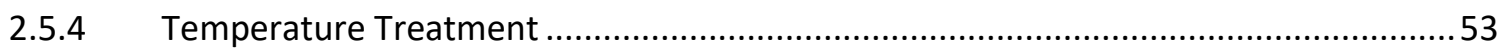

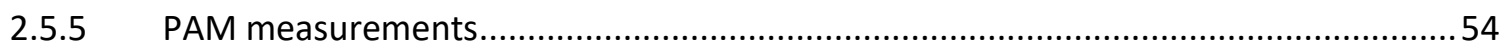

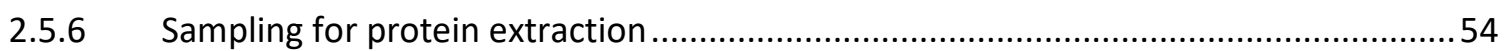

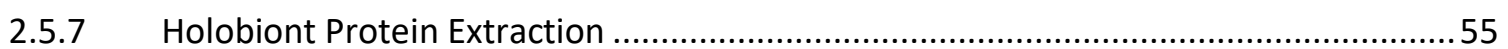

2.5.8 Western Blotting and Optical Density Analysis............................................................ 56

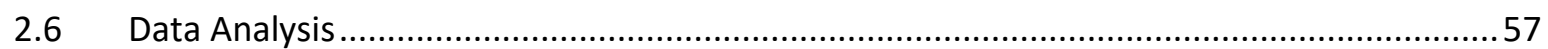

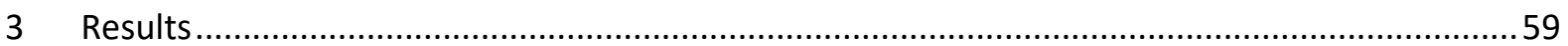

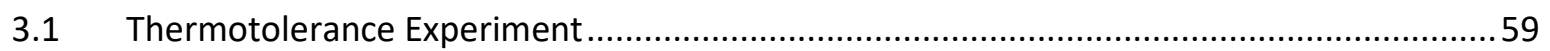

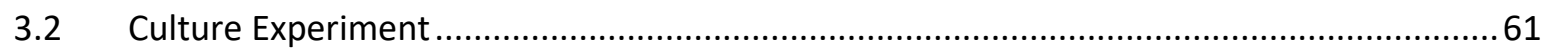

3.2.1 Influence of Temperature and Nutrients on Maximum Quantum Yield $\left(F_{v} / F_{M}\right)$.........62

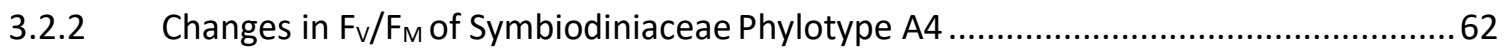

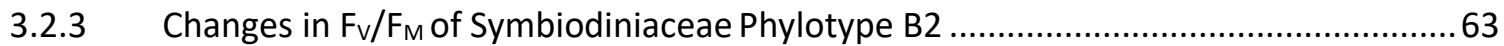

3.3 Influence of Temperature and Nutrient Regime on Fluorescence Yield as a Response to

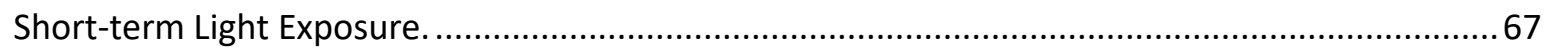

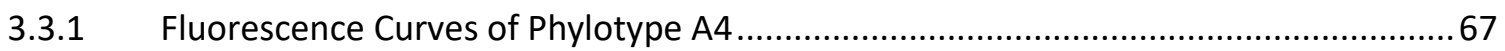

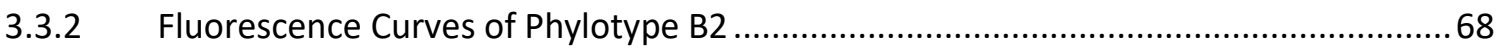

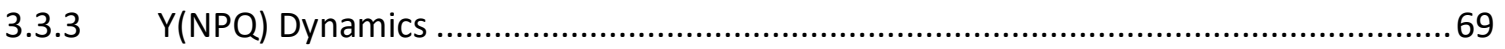

3.4 Influence of Temperature and Nutrients on D1 Protein Concentration.............................. 70 


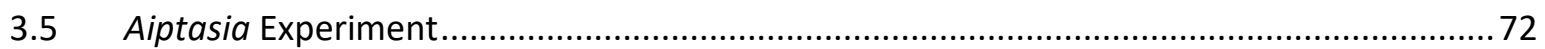

3.5.1 Influence of Feeding regime on growth of symbiotic Aiptasia .................................... 73

3.5.2 Influence of temperature and host feeding regime on $\mathrm{F}_{\mathrm{v}} / \mathrm{F}_{\mathrm{M}}$ of Symbiodiniaceae in Aiptasia 73

3.5.3 Influence of Temperature and Host Feeding regime on D1 protein concentration of

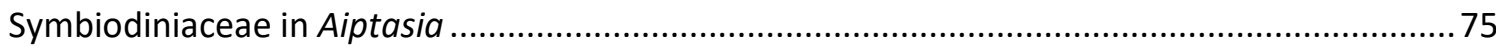

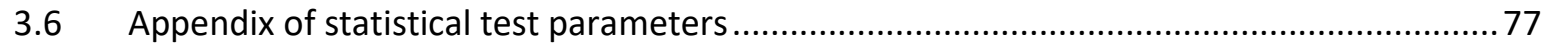

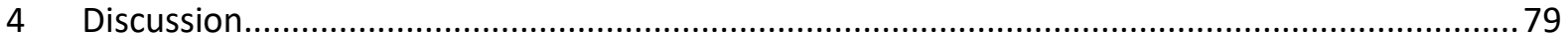

4.1 Importance of nutrient availability for photosynthetic health of cultured Symbiodiniaceae 79

4.1.1 Chloroplast protein synthesis and quantum yield 80

4.1.2 Evidence for physiological disparity between $A 4$ and B2 in response to light exposure 81

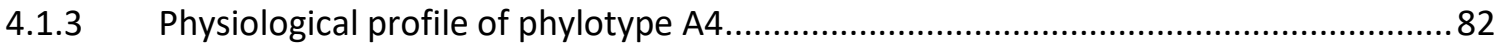

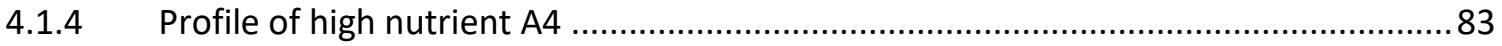

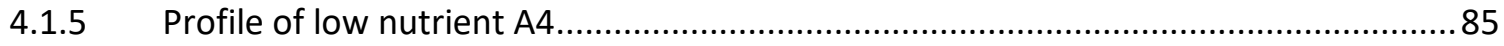

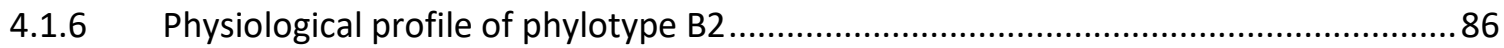

4.1.7 Relationship between thylakoid composition and D1 protein concentration...............88

4.2 Influence of host nutritional status on photosynthetic health of symbiotic

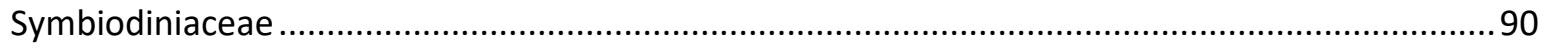

4.2.1 Influence of symbiosis on photosynthetic potential................................................. 90

4.2.2 Influence of Symbiodiniaceae type on host growth .................................................. 91

4.2.3 Influence of feeding and heat stress in hospite on D1 protein concentration ............. 91

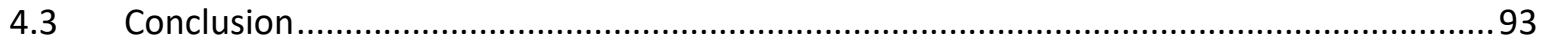

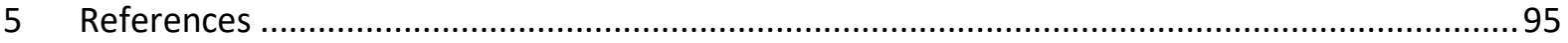




\section{Introduction}

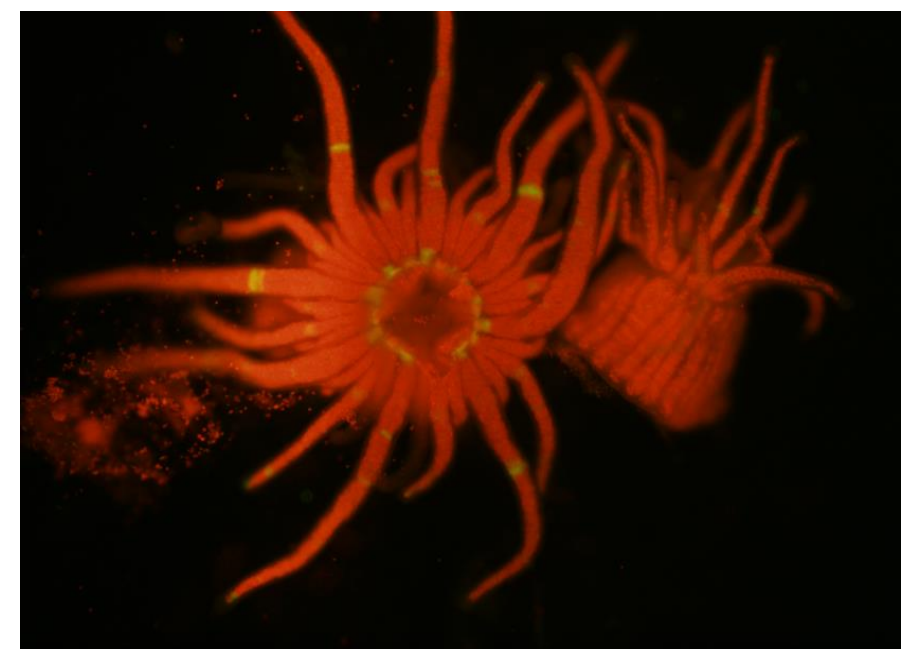

Fluorescence microscopy of the anemone Aiptasia pallida (Aiptasia) hosting Symbiodiniaceae (fluorescing red).

\subsection{Symbiosis and Coral reefs}

\subsubsection{Symbiosis}

Originally defined in 1878 by Anton de Bary as 'the living together of differently named organisms'(Oulhen et al, 2016), symbiosis is now recognised as essential to the development and survival of the majority of known organisms on earth (Gilbert et al, 2012). Observations of the myriad interactions between hosts and their respective symbionts have revealed that symbiotic relationships exist on a wide spectrum, with broad definitions developed to categorise general trends in symbiont/host relationships. Symbiosis may confer benefits to both partners equally (mutualism), benefit one partner only (commensalism) or benefit one partner at the expense of the other (parasitism), with some symbiotic associations shifting between these states as a consequence of changing environmental pressures or physiological condition of the participants (Douglas, 2008).

Relationships may be ectosymbiotic, where both partners live externally of each other, an example being the ocellaris clown fish (Amphiprion ocellaris) living amongst the tentacles of the anemone Heteractis magnifica, where it is protected from predation and is able to feed on parasites that may be otherwise detrimental to the anemone. Alternatively, the association may be endosymbiotic, where one partner resides within the body of the other, either transiently or permanently. An example of this is the bioluminescent bacterium Vibrio fischeri which inhabit light organs in the mantle of the Hawaiian 
bobtail squid (Euprymna scolopes), providing the squid with a means of camouflage and in turn gaining a hospitable environment in which to grow and reproduce (Widder, 2010).

A common and highly ecologically important example of endosymbiosis occurs in a variety of marine invertebrates, where autotrophic unicellular algae are enlisted into the tissues of the invertebrate to provide nutritive benefits via photosynthesis (Muscatine \& Cernichiari, 1969). While such relationships are found in a diverse range of marine animals (including anemones, tridacnid clams, sponges and foramniferans to name just a few), the most widely recognised endosymbiotic algal-invertebrate partnership is undoubtedly between the scleractinian (stony) corals of phylum Cnidaria and symbiotic dinoflagellates of family Symbiodiniaceae (LaJeunesse et al., 2018).

\subsubsection{Symbiodiniaceae and coral reefs}

The coral animal is a small polyp, similar in morphology to a sea anemone, which lives in colonies ranging from tens to thousands of individuals (Fig. 1.1) and all sharing a calcium carbonate $\left(\mathrm{CaCO}_{3}\right)$ skeleton of their own secretion. The symbiotic dinoflagellates are introduced into the gastrodermal cells of their coral hosts either through phagocytosis (horizontal transfer) or by being passed down from the host parent (vertical transfer) (Abrego et al., 2009; Baird et al., 2009). In the cell, the dinoflagellate inhabits the symbiosome, a vacuole specifically regulated by the host to accommodate the endosymbiont and to facilitate nutrient transfer between the symbiotic partners (Yellowlees et al., 2008), and from within which the symbiont initiates photosynthesis. Excess photosynthates (fixed carbon products of photosynthesis in the form of carbohydrates and fatty acids) are excreted out of the symbiont and passed on to the host.

Hermatypic (reef building) scleractinian corals obtain over $90 \%$ of their daily energy requirements from the photosynthates of their Symbiodiniaceae endosymbionts, an energy source which is crucial for growth, reproduction and accretion of the $\mathrm{CaCO}_{3}$ skeleton which makes up the reef structure on which they and countless other organisms live (Muscatine \& Cernichiari, 1969; Hughes et al., 2017). In turn, the symbiotic dinoflagellate benefits from nutrients found in the hosts waste products (particularly $\mathrm{CO}_{2}$, $\mathrm{NH}_{4}{ }^{-}$and $\mathrm{PO}_{4}{ }^{4-}$ ) as well as increased protection from grazers and a stable position in the water column suitable for photosynthesis (Yellowlees et al., 2008). 


\subsubsection{The value of reefs}

Coral reefs make up less than $0.5 \%$ of the worlds near-shore marine environment yet harbour the highest concentration of ocean biodiversity of any habitat, with over $25 \%$ of all marine life, including over 4,000 species of fish, dependent on coral reefs at some point in their life cycle (Costanza \& D'Arge, 1997; Carpenter et al., 2008). Besides their ecological importance, coral reefs are of significant economic value, providing an estimated US $\$ 30$ billion annually in net benefits to world economies through tourism, artisanal fishing and coastline protection (UN Environment ISU ICRI \& Trucost, 2018). The continued existence of the world's coral reefs relies fundamentally on the symbiotic relationship between scleractinian corals and their symbiotic dinoflagellates.
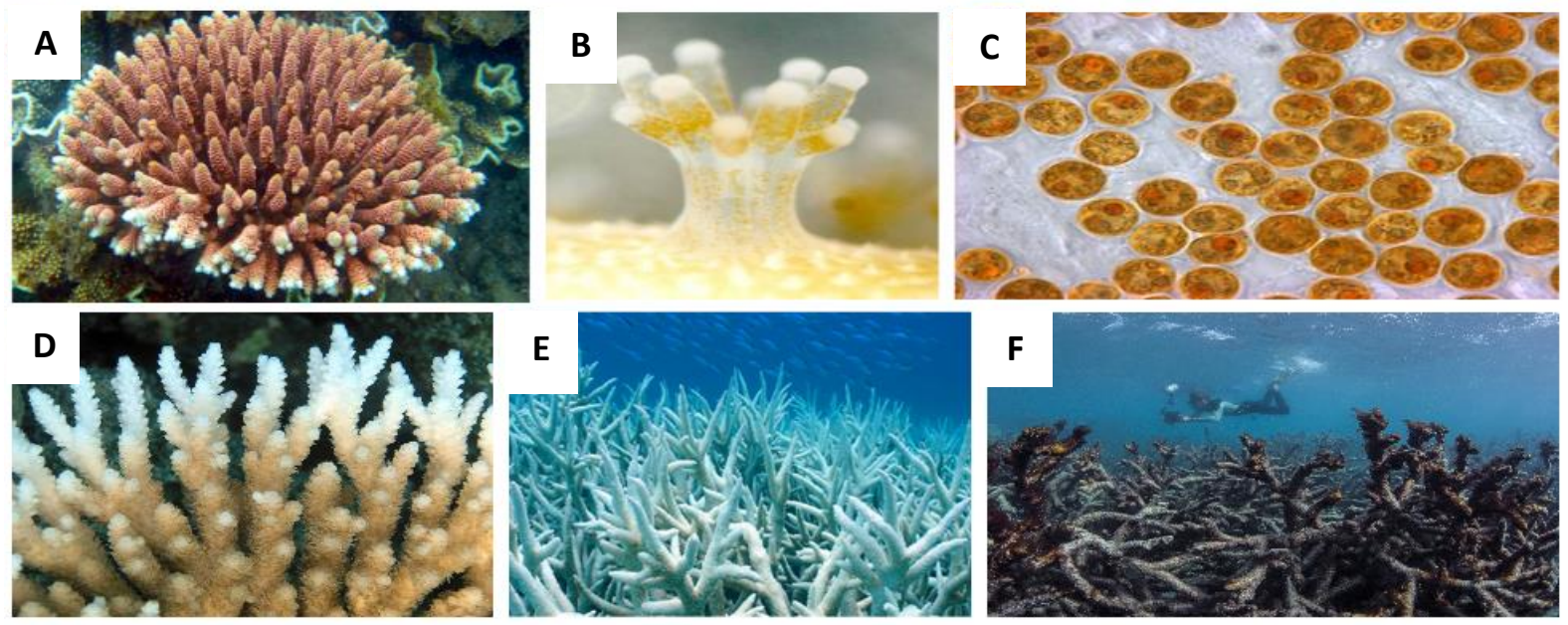

Fig 1.1 Coral symbiosis and bleaching. A) A staghorn coral colony (Acropora sp.) showing brown colouring due to the pigments of endosymbiotic algae (Symbiodiniaceae). B) Close up of an individual coral polyp showing endosymbionts within its gastrodermal cells, visible as green/brown spots. C) Free living, or cultured (without a host) Symbiodiniaceae. Each individual algal cell is approximately 7-10 $\mu \mathrm{m}$ in diameter. D) Coral in the process of bleaching. Lightening of the brown colouring is due to expulsion of endosymbionts. E) completely bleached corals. E) Dead corals being overgrown by algae and showing signs of erosion.

\subsubsection{Coral Bleaching}

When environmental conditions present significant physiological stress, such as prolonged exposure to elevated temperatures, strong irradiance and eutrophication, the cnidarian-dinoflagellate symbiosis risks breaking down (Bhagooli \& Hidaka, 2004; McCabe Reynolds et al., 2008; Thurber et al., 2014). Under such conditions, the algal symbionts may be expelled from the host in a process known as 'coral 
bleaching', so-called because the white $\mathrm{CaCO}_{3}$ skeleton becomes visible through the transparent coral polyp following the loss of algal pigments. With the breakdown of symbiosis, the coral becomes deprived of an essential energy source, and if symbiosis is not restored within a matter of days to weeks, the coral will eventually die.

Historically, bleaching events occurred naturally on many coral reefs due to localized environmental fluctuations, but the transience of such events generally allowed time for recovery of the affected areas by re-establishment of the symbiotic partnership (Glynn, 1993). However, with the increased pressure of warming sea surface temperatures fuelled by anthropogenic climate change, the frequency and severity of mass coral bleaching events in recent years has risen alarmingly, leading to massive coral die-off on reefs around the world (Hughes et al., 2017). Live coral cover in the Caribbean fell from 50\% in the 1970 's to just $10 \%$ in 2003 , while in 2008,407 of 845 described hermatypic coral species were listed as threatened or near-threatened by extinction (Carpenter et al., 2008; Jackson et al., 2012). With the continued increase in global and oceanic temperatures expected over the coming decades (Diffenbaugh et al., 2017), the threats facing this ecologically indispensable symbiotic partnership continue to mount.

\subsection{Symbiont diversity}

\subsubsection{Early Descriptions}

In 1881, the endosymbiotic dinoflagellates of family Symbiodiniaceae were originally taxonomically defined as members of the novel genus Zooxanthella (Krueger, 2017), of which there was considered to be only a single species. Kawaguti (1944) observed that the coccoid symbionts of the reef coral Acropora corymbosa, formed gymnodinioid-like zoospores when in culture, and he therefore assigned them to the dinoflagellate genus Gymnodinium. Freudenthal (1962) was the first to propose a lifecycle for gymnodinioid symbionts, cultured from the 'upside-down' jellyfish, Cassiopeia sp. Emphasising the importance of their symbiotic lifestyle, Freudenthal named these algae Symbiodinium microadriaticum, which also became variously known as Gymnodinium microadriaticum and 
Zooxanthella microadriaticum (Taylor, 1971; Loeblich \& Shirley, 1979), and were considered to be a ubiquitous and panmictic species within their marine hosts. However, it was Blank and Trench (1985) who showed for the first time, using morphometric analyses of the nuclei of 4 different strains of $S$. microadriaticum, that differences in chromosome numbers and chromosome volumes indicated that the taxonomic unit $S$. microadriaticum in fact represented a large species complex. Shortly after, three novel species of genus Symbiodinium were described by comparing differences in cell size and ultrastructure, including chromosome and pyrenoid numbers, as well as relative volumes of mitochondria and chloroplasts of algal isolates (Trench \& Blank, 1987).

Advances in genetic analysis techniques, in particular comparison of restriction fragment length polymorphisms (RFLPs) from nuclear genes encoding small subunit ribosomal DNA (18S-rDNA), further defined genus Symbiodinium (Rowan and Powers, 1991a). Sequence variation between individual isolates within genus Symbiodinium was comparable to that of orders of free-living dinoflagellates (Rowan \& Powers, 1991b), leading to the division of the genus into groups, or clades, loosely based on host taxa. Symbiodinium clades A, B, C (Rowan \& Powers, 1991b) and D (Carlos \& Baillie, 1999) were recognised as the predominant symbionts within scleractinian corals; clade E, of which there is a single known species (Lajeunesse \& Trench, 2000; LaJeunesse, 2001; Jeong et al., 2014) was found in the sea anemone Anthopleura elegantissima, while clades F (LaJeunesse, 2001), G (Pochon et al., 2001) H (Pochon et al., 2004) and I (Pochon \& Gates, 2010) are common in foraminifera. Eventually, a growing recognition of the high degree of genetic dissimilarity, existing not only at interbut also intracladal levels, drove discourse on the need to correctly recognise and formally name the many genetically distinct lineages of Symbiodinium to species level (Sampayo et al., 2009; Finney et al., 2010). A major systematic revision of the family Symbiodiniaceae by LaJeunesse et al., (2018) (fig. 1.2) led to the current taxonomic classification, where clades A-G have been reclassified as genera, with clade A now represented by genus Symbiodinium, clade B by Breviolum, clade C by Cladocopium, clade D by Durusdinium, clade E by Effrenium, clade F by Fugacium and clade G by Gerakladium 
Fig. 1.2 (Image redacted)

\subsubsection{Host specificity and Flexibility}

Unsurprisingly, the vast genetic diversity of Symbiodiniaceae translates into considerable phenotypic, and thus functional and ecological diversity across the range of known phylotypes. It is therefore reasonable to assume that Cnidarian hosts preferentially harbour symbionts that confer physiological benefits congruent with the ecology in which the host is found. While many dinoflagellates of the family Symbiodiniaceae show a generalist nature in host association, no species are ubiquitously generalist and some species are highly host and environment specific (Baker, 2003; Silverstein et al., 2012). Notably, while some host species exhibit a strong specificity to a particular genus of Symbiodiniaceae, they may also harbour additional 'background' symbionts of different species or genera (Rouzé et al., 2017; Bachtiar et al., 2019). This has important ecological consequences for the holobiont, in that the 
symbiont community composition within the host can in effect be 'shuffled' during periods of environmental change. This has been seen in corals that have undergone bleaching events, with the predominant pre-event symbiont phylotype being replaced by another, more thermotolerant phylotype post-event (Jones et al., 2008; Silverstein et al., 2015; Manzello et al., 2019; Thinesh et al., 2019). It is important to note that in these cases the phylotypes that became predominant post-event are considered to have already been present as a background type within the coral prior to the event. While uptake of new symbiont phylotypes in adult coral ('switching') in response to environmental change has been observed transiently in the lab (Coffroth et al., 2010), there is so far no indisputable evidence of it occurring in the field.

Symbiodiniaceae can be transmitted to the host directly from parent to offspring via eggs (vertical transmission) or acquired by aposymbiotic larvae/early recruits from the environment (horizontal transmission) (van Oppen, 2001). Transmission mode is correlated with the reproductive strategy of the host. Around $90 \%$ of brooding corals transmit vertically and $\sim 80 \%$ of spawning corals transmit horizontally, with hermaphroditic and gonochoristic spawners representing the vast majority of known scleractinian coral species ( 84\%) (Baird et al., 2009). Corals that acquire symbionts through vertical transmission may benefit from the evolutionary success of the host-symbiont fitness of prior generations, particularly with regards to thermotolerance and resistance to bleaching(Swain et al., 2018). However, vertical transmission is also correlated with high host specificity (Fabina et al., 2012), which may limit the adaptive capacity of vertical transmitters when exposed to environmental change. On the other hand, horizontal transmission allows for acquisition of physiologically novel symbiont phylotypes, a potential advantage in transitional environments.

\subsubsection{Thermotolerance}

Prolonged thermal stress results in loss of photosynthetic functions within many phototrophic organisms, including Symbiodiniaceae (Berry \& Bjorkman, 1980; Iglesias-Prieto et al., 1992). This can lead to photoinhibition of the chloroplast and, in the case of the coral holobiont, may result in temperature induced bleaching (Bhagooli \& Hidaka, 2004; Baker et al., 2008; Díaz-Almeyda et al., 2017; Sully et al., 2019; Romero-Torres et al., 2020). Degrees of thermotolerance (that is, the ability to 
withstand increased ambient temperatures without loss of photosynthetic function) vary significantly between Symbiodiniaceae types. It was originally supposed that thermotolerance was correlated with phylogenetic divergence within the family, such that all members of a certain genera (formally clades) were inherently more or less thermotolerant than those of another genera (Rowan et al., 1997; Warner et al., 1999). It has since been recognised that Symbiodiniaceae show high intra- and inter-generic diversity in thermotolerance, as well as some degree of intraspecific diversity (i.e. between strains of the same species) (Díaz-Almeyda et al., 2017; Swain et al., 2017; Mansour et al., 2018).

There is now general acceptance that specific Symbiodiniaceae species, such as Durusdinium trenchii and Cladocopium thermophilum, exhibit high resistance to thermal stress, relative to thermally sensitive phylotypes such as (ITS2) type C7 (Hume et al., 2015; Kemp et al., 2015; Silverstein et al., 2017).

The exact physiological traits that confer thermotolerance to individual Symbiodiniaceae phylotypes is a topic of ongoing research, however relative thermotolerance in Symbiodiniaceae is likely to rely on a combination of factors, including (but not limited to) the ability of the symbiont to adapt to fluctuations in incident irradiance and its efficiency in dealing with oxidative stress (Suggett et al., 2008; Das et al., 2015; Lesser, 2019).

Importantly, symbiotic dinoflagellates that show high thermotolerance are often less favourable to their cnidarian hosts, as these symbionts tend towards elevated levels of photosynthate and organic nutrient retention (Stat \& Gates, 2011; Baker et al., 2018). Thermotolerant Symbiodiniaceae are often found in health compromised corals that have previously undergone thermal stress, where their symbiotic association tends towards parasitism. This may be due to thermotolerant species outcompeting thermally sensitive species at elevated temperatures, with the host retaining the thermally tolerant species at the cost of lowered photosynthate translocation and a resultant decrease in host growth- and reproductive-rate as well as reduced $\mathrm{CaCO}_{3}$ accretion. This may have important implications concerning optimal cnidarian-dinoflagellate partnerships as sea surface temperatures continue to rise (Gibbin et al., 2018). 


\subsection{Nutrient Exchange}

\subsubsection{Carbon Exchange}

Metabolic exchange during coral-dinoflagellate symbiosis is central to the ecological success of the partnership. More than $60 \%$ of carbon photosynthetically fixed by the symbiont may be translocated to the host (Taylor, 1969), providing energy for respiration, growth and reproduction. This high percentage of photosynthate translocation is a consequence of a chemical signal provided by the host, loosely termed 'host release factor' (HRF) (Muscatine, 1967). HRF greatly enhances photosynthate output in the symbiont, as free-living dinoflagellates would typically release less than $5 \%$ of their fixed carbon into the surrounding medium. The exact properties of HRF are largely unknown, but it's involvement has been confirmed in a range of cnidarian-dinoflagellate symbioses (Sutton \& Hoegh-Guldberg, 1990; Grant et al., 1997; Davy \& Cook, 2001).

Inorganic carbon $(\mathrm{Ci})$ is delivered to the symbiont in the form of $\mathrm{CO}_{2}$, as a waste product of host respiration (Fig. 1.3). However, to facilitate the increased rate of photosynthesis required of the dinoflagellate in hospite (within the host) a higher concentration of $\mathrm{CO}_{2}$ is needed than is typically found in sea water. The host remedies this via the enzyme carbon anhydrase (CA), which catalyses the conversion of bicarbonate $\left(\mathrm{HCO}_{3}^{-}\right)$, a compound naturally abundant in seawater, into $\mathrm{CO}_{2}$, thus greatly increasing the concentration of $\mathrm{CO}_{2}$ in the symbiosome (Weis \& Reynolds, 1999; Ganot et al., 2011). $\mathrm{CA}$ also facilitates biomineralization in cnidarians, increasing the accretion rate of $\mathrm{CaCO}_{3}$ into the skeletal structure (Le Goff et al., 2016).

\subsubsection{Nitrogen Exchange}

Access to nitrogen $(\mathrm{N})$ is crucial for all living organisms due to its functional role in a host of organic molecules, including nucleic acids, amino acids and adenosine triphosphate (ATP). In free living Symbiodiniaceae, ammonium is assimilated into the cell directly from seawater by the glutamine synthetase/glutamine 2-oxoglutarate amidotransferease (GS/GOGAT) cycle (Roberts et al., 2001). This produces glutamate, a key amino acid which also serves as a precursor to synthesis of DNA, RNA, pigments and certain lipids (Rahav et al., 1989; Xiang et al., 2020). In hospite the symbiont loses direct 
access to seawater, but may still receive ammonium through the cell walls of the host via seawater filling the host's gastrovascular cavity (Pernice et al., 2012). The symbiont can also incorporate nitrate $\left(\mathrm{NO}_{3}\right)$ directly from seawater, converting it to ammonium before further metabolism via GS/GOGAT, but the process is less metabolically efficient than direct ammonium assimilation (Ferrier-Pagè et al., 2015).

Confounding all this however is 'Darwin's Paradox', which concerns the fact that corals flourish in notoriously oligotrophic water (lacking in nutrients) and, despite the abundance of life, coral reefs have been referred to as 'marine deserts', due to the extremely low concentration of dissolved organic and inorganic matter (van Oevelen et al., 2013; Cui et al., 2019). This means that the dinoflagellate symbiont

Fig. 1.3 (Image Redacted)

relies almost exclusively on ammonium supplied by the host to meet its $\mathrm{N}$ requirements, which mostly comes in the form of waste ammonium from digested particulate matter consumed heterotrophically by the host (McAuley \& Cook, 1994).

Nitrogen and amino acids are therefore tightly recycled within the cnidarian-dinoflagellate symbiosis and, unsurprisingly, symbiotic dinoflagellates in hospite are generally considered to be nitrogen limited (Muscatine et al., 1989; Cook et al., 1992; Titlyanov et al., 2000; Davy et al., 2012; Xiang et al., 2020). This, however, is not only due to oligotrophic seawater, as it is also well understood that cnidarian hosts 
actively limit $\mathrm{N}$ availability to their endosymbionts as a means of controlling their growth and reproductive rate (Rädecker et al., 2015; Cui et al., 2019). If allowed to grow at the same rate as the host cells, the symbionts would not only eventually overwhelm the host, but also consume large amounts of fixed carbon through the building of proteins and lipids, leaving less for the hosts metabolic demands. Wang and Douglas (1998) showed that the model symbiotic anemone Aiptasia pallida employed fixed carbon to assimilate ammonium through the ATP-dependent amination of glutamate to glutamine, via GS, thus directly competing with the symbiont for access to $\mathrm{N}$ through ammonium. Xiang et al., (2020) showed that as algal populations in Aiptasia increased and more fixed carbon became available, assimilation of ammonium in the host also increased, effectively slowing symbiont growth.

Therefore, $\mathrm{N}$ availability in hospite is of considerable metabolic importance for the cnidariandinoflagellate symbiosis. As the main goal of the host is to encourage optimal photosynthetic output from the symbiont, it is crucial that the symbiont is able to make optimal use of available nutrients to facilitate photosynthate production. This may include preferential investment of $\mathrm{N}$ into synthesis of essential chloroplastic metabolites and proteins, such as D1 protein, which will be discussed shortly.

\subsection{Concepts of Photophysiology}

At the heart of the symbiotic partnership between Symbiodiniaceae and its cnidarian host is the process of photosynthesis. Photoinhibition, or the breakdown of photosynthesis, is a precursor to symbiont expulsion, and thus coral bleaching (Jones et al., 1998; Warner et al., 1999; Smith et al., 2005; Wangpraseurt et al., 2017; Carballo-Bolaños et al., 2019) and therefore a large body of work has been devoted to mechanisms of photoinhibition in Symbiodiniaceae and strategies that protect against it (Hill et al., 2014; Dang et al., 2019a). Accordingly, it is necessary to understand the principles of the photosynthetic system and the mechanisms behind certain photophysiological pathways. 


\subsubsection{The Chloroplast}

The success of the holobiont partnership relies fundamentally on the ability of the symbiont to efficiently carry out photosynthesis within the gastroderm of the host. Like all eukaryotic photoautotrophs, Symbiodiniaceae contain chloroplast organelles, made up of a pair of lipid bilayers enclosing a stromal space, in which floats a protein-embedded thylakoid membrane with a central lumen. The chloroplast harvests photons in the thylakoid membrane through the 'light-dependent reaction' of photosynthesis. The energy gained from these photons is used to drive an electron transport chain (ETC) which ultimately leads to the reduction of nicotinamide adenine dinucleotide phosphate $\left(\mathrm{NADP}^{+}\right)$to NADPH, used as a reducing agent in the so-called 'dark reaction' of the Calvin-Benson cycle (CBC) (Bassham et al., 1950). The CBC is responsible for fixing inorganic carbon into biologically consumable carbohydrates in the stroma, employing the enzyme ribulose-1,5-bisphosphate carboxylase/oxygenase (Rubisco).

A second product of the light-dependent reaction is adenosine triphosphate (ATP), the synthesis of which is driven by a $\mathrm{pH}$ gradient formed across the thylakoid membrane (Fig. 1.4a). The gradient is caused by a build-up of $\mathrm{H}^{+}$ions in the lumen as a result of water oxidation by the oxygen evolving complex found in photosystem II (PSII), as well as via transfer of electrons from PSII to the electron acceptor plastoquinone,

\subsubsection{The Photosystems and the electron transport chain}

The thylakoid embedded proteins of the chloroplast are grouped into multi-subunit protein complexes, each of which contributes uniquely to the electron transport chain (ETC). PSII, the first major protein complex in the light dependent process of photosynthesis, is made up of a heterodimer of the transthylakoid proteins D1 and D2, which are bound to the oxygen evolving complex (OEC) and flanked by the transmembrane chlorophyll proteins CP47 and CP43 (Bricker \& Frankel, 2002), to which hundreds of 'antennae' pigment molecules are bound in a precise, 
Fig 1.4. (Image Redacted)

yet potentially motile, configuration (Fig. 1.4a). The antennae pigments, known collectively as the light harvesting complex (LHC), readily absorb photons with wavelengths between $400 \mathrm{~nm}$ and $670 \mathrm{~nm}$, the energy of which kicks an electron in the pigment molecule into a higher, less stable orbit. This energy is propagated along a series of neighbouring pigments via fluorescence resonance energy transfer (FRET), until it reaches the reaction centre of PSII (RCII) (Fig. 1.4b) At RCII the energy is passed to the chlorophyll cluster P680 which undergoes charge separation to become $\mathrm{P} 680^{+}$, passing on an electron to the primary electron acceptor quinone-A ( $\mathrm{Qa}$, reduced $\left.\mathrm{Qa}^{-}\right)$, via pheophytin (Phe), both of which are bound within the D1-D2 heterodimer (Fig. 1.4a). P680+ is then reduced back to P680 after accepting an electron from a tyrosine molecule (Yz), which is itself reduced by an electron cleaved from water via the OEC. The reduced $\mathrm{Qa}^{-}$passes its electron to the loosely bound secondary electron acceptor quinone- $\mathrm{B}(\mathrm{Qb}) . \mathrm{Qb}$ requires two electrons to become fully reduced to $\mathrm{Qb}^{2-}$, therefore two $\mathrm{Qa}^{-}$are needed to fully reduce a single $\mathrm{Qb}$ molecule, requiring at least 2 turnovers of PSII. The fully reduced 
$\mathrm{Qb}^{2-}$ binds with two protons $\left(\mathrm{H}^{+}\right)$from the stroma and is released into the thylakoid membrane where it becomes part of the free living plastoquinone pool. Protonated plastoquinone diffuses through the thylakoid membrane until it reaches cytochrome $b_{6} / f$, where it attaches to a binding site on the lumenal side of the membrane, releasing its protons into the lumen and passing the electrons on to a plastocyanin molecule which then passes them on to the chlorophyll pair P700 found in the reaction centre of photosystem I (RCI). The end result is a transfer of electrons from P700 to the terminal electron acceptor ferrodoxin $(\mathrm{Fd})$. The reduced form of ferrodoxin is now available to reduce $\mathrm{NADP}^{+}$to NADPH via the ferrodoxin-NADP-oxidoreductase enzyme (FNR), with NADPH able to be used in the dark reactions of the $\mathrm{CBC}$ for the fixation of carbon. Alternatively, a series of non-carbon reducing paths are available to the electrons in the reduced ferrodoxin, including the Mehler reaction, photorespiration, nitrogen reduction and cyclic electron flow.

\subsubsection{Photosystem II and the D1/D2 heterodimer}

While the PSII complex is composed of over twenty-five subunits, the light induced electron transfer reactions occur solely within the D1/D2 heterodimer, which binds all the co-factors involved in photosynthetic water oxidation (Nixon et al., 2006) (Fig. 1.4a). The D1 protein is a rapid turnover protein, in that it is specifically degraded under illumination in vivo and is continuously re-synthesized de novo in the thylakoid membrane of the chloroplast (Mattoo et al., 1981; Mattoo et al., 1984). Under conditions of excess irradiance, degradation of D1 may outpace de novo synthesis, resulting in a net loss of D1 content and photoinactivation of the PSII reaction centre (Allakhverdiev \& Murata, 2004). Accumulation of photoinactivated RCIIs leads to photoinhibition, defined as the significant and longterm loss of photosynthetic yield within the chloroplast.

It has been long understood that photoinactivation of PSII is an inevitable result of exposure to visible and UV light (Powles, 1984), and that photoinactivation occurs when absorbed light exceeds the capacity of the chloroplast to utilize the excitation energy (Chow \& Aro, 2006). All other things being equal, combinations of irradiance and duration of illumination that give the same photon exposure (i.e., total incident photons $\mathrm{m}^{-2}$ ) will produce the same extent of photoinactivation (Jones \& Kok, 1966; Park et al., 1995; Tyystjarvi \& Aro, 1996). For this reason, much work on photoinhibition has concentrated 
on identifying the potential agents of D1 degradation as a function of photon induced charge separation within PSII.

\subsubsection{Mechanisms of Photoinhibition}

Rates of photoinactivation may increase as a function of the steady-state redox potential of various PSII components (De Las Rivas et al., 1992; Keren et al., 1997; Mellis, 1999). The model known as acceptorside inhibition posits that as the steady-state redox potential of the primary electron acceptor pool shifts to a reduced state $\left(\mathrm{Qa}_{-}>\mathrm{Qa}^{-}\right)$, electron flow from $\mathrm{Phe} e^{-}$to $\mathrm{Q}$ a is slowed due to a lack of available oxidised downstream acceptors. Under these conditions, Phe ${ }^{-}$can undergo charge recombination with P680, with a high probability of forming the P680 triplet state (Giorgi et al., 1990). Triplet P680 is quenched efficiently by $\mathrm{O}_{2}$, thereby generating the highly reactive oxygen species (ROS) singlet oxygen $\left({ }^{1} \mathrm{O}_{2}\right)$, which readily reacts with protein (i.e. D1) and/or pigment components in the immediate vicinity, causing irreversible functional and structural damage (Vass et al., 1992). However, this model is inconsistent with studies which demonstrate that inhibition of electron transport from $\mathrm{Qa}^{-}$to $\mathrm{Qb}$ has no effect on the rate of photodamage of PSII (Komenda \& Masojídek, 1998; Allakhverdiev et al., 2005). Alternatively, donor-side inhibition assumes that reduction of $\mathrm{P} 680^{+}$is slowed, potentially due to overacidification of the thylakoid lumen under high light (Krieger \& Weis, 1993). This is proposed to have the effect of reversibly releasing $\mathrm{Ca}^{2+}$ from the OEC and temporarily preventing electron transfer from $\mathrm{H}_{2} \mathrm{O}$ to P680+ (via tyrosine, $\mathrm{Tyr}_{\mathrm{z}}^{-}$) (Boussac \& Rutherford, 1992). P680 ${ }^{+}$, being the most powerful oxidant involved in photosynthesis due to its ability to extract electrons from otherwise stable water molecules (Chow \& Aro, 2006), will react readily with neighbouring molecules if not immediately reduced through charge recombination (Bumann \& Oesterhelt, 1995). Thus, the extended life-time of $\mathrm{P}_{680}+$ under the above conditions would result in oxidation of key PSII components, particularly the D1 protein within which it is bound (Anderson et al., 1998).

A more recently proposed mechanism sees degradation of D1 as a secondary event in a process known as the two-step model (Hakala et al., 2004; Ohnishi et al., 2005). In this scenario photodamage to PSII occurs initially at the water splitting site of the OEC, specifically through photon induced displacement of a $\mathrm{Mn}^{2+}$ ion from the Mn-Ca cluster (Zavafer et al., 2015). As in the donor side model, electron flow 
from $\mathrm{H}_{2} \mathrm{O}$ to $\mathrm{P}_{680^{+}}$is disrupted and oxidative damage occurs in the D1 protein. Notably however, the two step model disregards redox potential within PSII as being the rate limiting entity in photoinactivation. Instead, photodamage to the OEC initiates D1 degradation at a rate-constant proportional to incident photon flux (Hakala et al., 2004), a scenario strongly supported by observations of rates of photoinactivation under moderate light (Park et al., 1995; Tyystjarvi \& Aro, 1996; Tyystjärvi, 2008). Photoinhibition of the chloroplast then occurs as synthesis de novo of replacement D1 is inhibited at the translational level by ROS (Nishiyama et al., 2006). It is well understood that when electron flow rate from PSII increases beyond the $\mathrm{CO}_{2}$ fixation capacity of the $\mathrm{CBC}$, excess electrons can be diverted through an alternative sink in PSI. Known as the water-water cycle, this process uses electrons cleaved from water in PSII to reduce $\mathrm{O}_{2}$ to the superoxide radical $\left(\mathrm{O}_{2}^{-}\right)$(Mehler, 1951), which is disproportionated to $\mathrm{H}_{2} \mathrm{O}_{2}$ via the catalyst superoxide dismutase (SOD) (Asada, 2006), with $\mathrm{H}_{2} \mathrm{O}_{2}$ then reduced to $\mathrm{H}_{2} \mathrm{O}$ by ascorbate, a reaction catalyzed by ascorbate peroxidase (APX). Thus, efficient ROS scavenging by SOD and APX in PSI is essential for suppressing the build-up of naturally occurring oxidative stressors. Under conditions where electron flow from PSI to the terminal ferrodoxin is inhibited, such as when the concentration of NADPH increases beyond the capacity of the CBC to utilize it, electrons diverted through the water-water cycle may increase levels of $\mathrm{O}_{2}{ }^{-}$and $\mathrm{H}_{2} \mathrm{O}_{2}$ at a rate beyond which SOD and APX can respectively scavenge them. $\mathrm{H}_{2} \mathrm{O}_{2}$ has been demonstrated to inhibit D1 synthesis at the translational level by oxidising the translation factor EF-Tu, responsible for the elongation step in the translation of PsbA mRNA, which encodes the D1 protein (Nishiyama et al., 2001; Takahashi \& Murata, 2008; Jimbo et al., 2018). Hence, according to this model, photoinhibition is the result of a reduced rate of D1 synthesis, as opposed to an increased rate of D1 degradation. This model, however, may not hold true for chloroplasts subject to non-irradiative stress only (Hill et al., 2011). 


\subsubsection{Irradiative tolerance}

By necessity, Symbiodiniaceae-harbouring scleractinian corals may only inhabit areas of the ocean where downwelling sunlight is sufficient for photosynthesis. As such, the biogeographic range of hermatypic corals is dictated by factors which affect the amount of incident irradiance available to the symbiont, such as depth, water turbidity and weather. In general, coral reefs are found in relatively shallow ( $<100 \mathrm{~m}$ deep) tropical and sub-tropical areas, where a warm, stratified water-column results in clear, oligotrophic surface waters, allowing for minimal absorption and dispersal of downwelling light. However, these environments also present significant spatial and temporal variations in light intensity, to which both the coral host and the symbiont must adapt in order to efficiently utilise photosynthetically active radiation (PAR) (Warner \& Suggett, 2016).

The primary spatial gradient for light intensity on a coral reef is depth, as light is naturally absorbed and scattered relative to how far it travels through the water column (Innis et al., 2018). Zonation of Symbiodiniaceae phylotypes as a function of depth has been observed in a number of coral species (Rowan \& Knowlton, 1995; Toller et al., 2001; Kemp et al., 2015; Innis et al., 2018), indicating that the dominant symbiont within a coral host may be of a phylotype most suited to the ambient irradiance at any given depth. A similar pattern has been observed on a macro-scale, where photo-tolerant Symbiodiniaceae phylotypes have been found exclusively on dorsal areas of coral colonies and photosensitive types located laterally, or even ventrally, within the same colony (Rowan et al., 1997; Kemp et al., 2015). This has been interpreted as an indication that photoadaptation to incident irradiance is set according to genotypic constraints in the cellular functioning of the symbiont (Warner \& Suggett, 2016). However, regardless of depth, incident irradiance fluctuates considerably, and often stochastically, on a range of temporal scales. Changes in cloud cover, water turbidity and daily and seasonal variations in sun intensity all dramatically alter the local light environment of the coral reef. For this reason, members of Symbiodiniaceae (and indeed most known photoautotrophs) have developed a suite of physiologically regulated protective measures to allow them to acclimate to short- and long-term fluctuations in incident irradiation (Niyogi \& Truong, 2013; Goss \& Lepetit, 2015). These photoacclimation strategies, known as non-photochemical quenching (NPQ), allow the symbiont to limit overexcitation of photosynthetic pigments and the electron transport chain under high light, while 
still efficiently maintaining photosynthesis when light levels drop. In the context of the current study, the most relevant form of NPQ is energy quenching $(q E)$, or the dissipation of excess photonic energy as heat. This process is regulated by the $\mathrm{pH}$ gradient $(\Delta \mathrm{pH})$ formed across the thylakoid membrane during light driven photosynthesis. High-light induced acidification of the thylakoid lumen triggers reversible de-epoxidation of xanthophyll pigments in LHCII, causing antenna pigment aggregation followed by thermal deactivation of excited-state LHCII pigments (Jahns \& Holzwarth, 2012; Ruban et al., 2012). Under these conditions, excess excitation energy is lost before it reaches RCII, limiting photo-oxidative damage to PSII. Implementation of $q E$ occurs within seconds of high $\Delta \mathrm{pH}$ formation and relaxes again within tens of seconds of $\Delta \mathrm{pH}$ returning to base levels(Demmig-Adams \& Adams, 2006), allowing the autotroph to rapidly acclimate to sudden fluctuations in incident irradiance. $q E$ has been seen to contribute $\sim 60 \%$ of total NPQ in Symbiodiniaceae exposed to high light environments (Hill et al., 2005).

\subsection{Aims and objectives of the study}

The continued existence of coral reefs relies fundamentally on the success of cnidarian-dinoflagellate symbiosis, yet this partnership is under multiple threats, not least from increasing sea surface temperatures due to anthropogenic climate change. It is understood that Symbiodiniaceae that show elevated thermotolerance may confer a degree of protection to their hosts in warming seas, but often at

the cost of balanced nutrient exchange between the two partners. Maintaining high levels of photosynthesis requires allocation of nutrients to chloroplastic metabolism, the rate of which may differ between symbionts of differing thermal sensitivity. Therefore, to understand the mechanisms which play a role in how coral and their endosymbionts will adapt to rising sea temperatures, it is necessary to understand the physiological changes caused by thermal stress in both the symbiont and the host and how these changes may affect nutrient allocation during photosynthesis. 
The aim of this study will be to investigate how thermotolerance to photoinhibition in Symbiodiniaceae is connected to nutrient availability and to what extent these factors affect the metabolic requirements of the host. This will be accomplished by addressing the following two questions:

1) Is thermotolerance in Symbiodiniaceae a consequence of more efficient nutrient use in photosynthesis in low nutrient environments?

$\mathrm{H}_{0}$ : Thermotolerant and thermally sensitive Symbiodiniaceae will synthesize equal concentrations of the chloroplast D1 protein under heat and nutrient stress.

$\mathrm{H}_{1:}$ Thermotolerant Symbiodiniaceae will synthesize higher concentrations of D1 protein under heat and nutrient stress than thermally sensitive species.

2) Is the growth and thermal tolerance of a host influenced by the efficiency of Symbiodiniaceae to synthesize photosynthetic proteins in a low nutrient environment?

$\mathrm{H}_{0}$ : Host growth-rate and thermotolerance will not be affected by the ability of the symbiont to synthesise D1 protein in a low nutrient environment.

$\mathrm{H}_{1}$ : The host will show improved growth and thermotolerance when harbouring Symbiodiniaceae that are able to efficiently synthesize D1 protein in a low nutrient environment. 


\section{Methods}

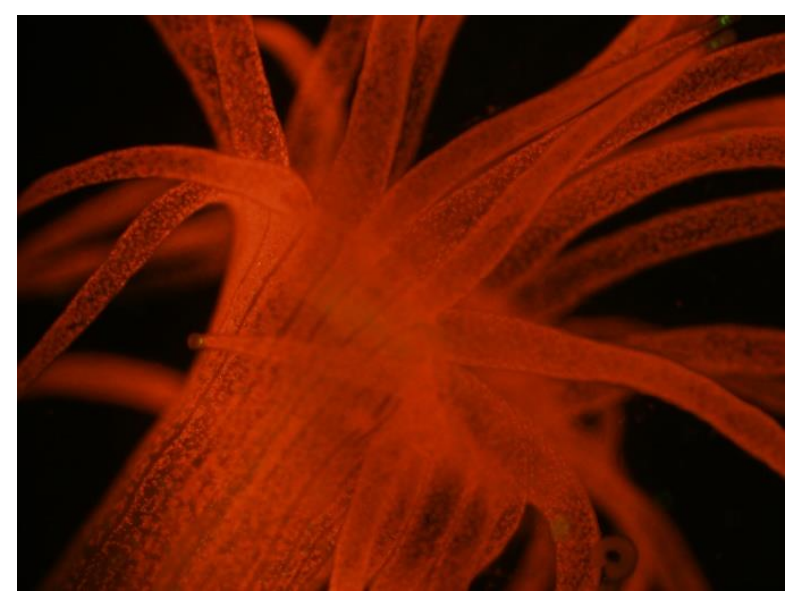

Aiptasia hosting B. minutum

\subsection{Thermotolerance}

A preliminary trial was run to select organisms based on their apparent thermotolerance, which were then to be used in the subsequent experiments.

\subsubsection{Experimental Organisms}

Five Symbiodiniaceae phylotypes were selected for use in preliminary heat tolerance experiments, drawn from a stock library of cultures maintained in an incubator (Biosyn $6000 \mathrm{CP}$, Contherm) kept for $10+$ years at $25^{\circ} \mathrm{C}$, under an irradiance of $\sim 55 \mu \mathrm{mol}$ photons $\mathrm{m}^{-2} \mathrm{~s}^{-1}$ (provided by $4 \mathrm{x}$ TLD $18 \mathrm{~W} / 840 \mathrm{Cool}$ White fluorescent lights) on a $12 \mathrm{~h}: 12 \mathrm{~h}$ light:dark cycle. Individual phylotypes were selected based on their relative thermotolerance as reported by Swain et al. (2017), with the intention of building an experimental sub-set representing phylotypes of high, medium and low thermotolerance. Four of the cultures were originally isolated from geographically distinct populations of the sea anemone Aiptasia pallida, (commonly referred to as 'Aiptasia), and one culture from a species of the coral Turbinaria (Table 1). All phylotypes will from here on be referred to by either their taxonomic nomenclature, or, if one is forthcoming, then by their ribosomal DNA internal transcribed spacer region 2 (ITS2) sequence type, a genetic sequence used to identify Symbiodiniaceae to the sub-species level.

Approximately $500 \mu \mathrm{l}$ of each Symbiodiniaceae culture was inoculated into flasks containing $\sim 250 \mathrm{ml}$ of artificial seawater (ASW) (Aquaforest, Poland), enriched with f/2 medium (Algaboost, Australia). 
The flasks were incubated at $25.5^{\circ} \mathrm{C}$, and exposed to an irradiance of $\sim 40 \mu \mathrm{mol}$ photons $\mathrm{m}^{-2} \mathrm{~s}^{-1}$ provided by 4 x TLD $18 \mathrm{~W} / 840$ Cool White fluorescent lights on a $12 \mathrm{~h}: 12 \mathrm{~h}$ light : dark cycle. A $100 \mathrm{ml}$ volume of $\mathrm{f} / 2$ enriched ASW was replaced in each flask every two weeks to maintain growth in the exponential phase.

Table 1. List of experimental culture phylotypes. Taxonomic Name: Genus, species, N/A = Not yet described; ITS 2 Type: Phylotypic classification based on genetic sequencing of the ribosomal DNA ITS2 region. First letter indicates genus (formally clade), $A=$ Symbiodinium, $B=$ Breviolum, $C=$ Cladocopium, $D=$ Durusdinium; Host Organism: Organism from which culture was originally isolated; Geographic Origin: Region and country where host organism was collected.

\begin{tabular}{|c|c|c|c|}
\hline Taxonomic Name & ITS2 Type & Host Organism & Geographic Origin \\
\hline N/A & A4 & Aiptasia & Florida, USA \\
\hline Breviolom minutum & B1 & Aiptasia & Florida, USA \\
\hline N/A & B2 & Aiptasia & Kanehoe Bay, Hawaii \\
\hline N/A & C1 & Turbinaria sp. & Lord Howe Island, Australia \\
\hline Durusdinium trenchii & D1a & Aiptaisia & Okinawa, Japan \\
\hline
\end{tabular}

\subsubsection{Experimental Set-up}

After approximately six weeks incubation, biomass from individual stock flasks was transferred to glass screw cap jars containing $15 \mathrm{ml} / 2$ medium ( $\mathrm{n}=6$ bottles per phylotype), with a final cell density of

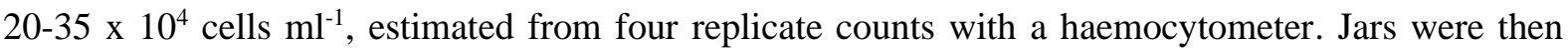
randomly assigned to one of two treatments; Control (C), with cultures maintained in a water bath held at $26^{\circ} \mathrm{C}\left( \pm 0.5^{\circ} \mathrm{C}\right)$, or Thermal $(\mathrm{T})$, with cultures subjected to a gradual increase in temperature of $1^{\circ} \mathrm{C}$ every two days, starting from an initial $26^{\circ} \mathrm{C}$ ( $\mathrm{n}=3$ per phylotype per treatment). The temperature of each water bath was monitored by a HOBO Pendant (UA-002-08, Onset) temperature/light digital data recorder, set to take readings every $15 \mathrm{~min}$. Light exposure for both groups was maintained $120 \mu \mathrm{mol}$ 
photons $\mathrm{m}^{-2} \mathrm{~s}^{-1}$, provided by $4 \times \mathrm{x} 5-54 \mathrm{~W}$ overhead fluorescent tubes, on a $12 \mathrm{~h}: 12 \mathrm{~h}$ light : dark cycle, which was maintained throughout the experiment. The selected irradiance levels approximately reflected those found on a low latitude reef 2-3 hours after sunrise, at which time corals are at their most photosynthetically active, while employing minimal photoprotective mechanisms (Hill \& Ralph, 2005). It was reasoned that such lighting conditions would exclude the effects of long-term light stress as a confounding variable in the experiment, while allowing for a measurable photochemical response to the thermal regime.

\subsubsection{Photosynthetic performance and thermotolerance}

Maximum quantum yield $\left(\mathrm{F}_{\mathrm{V}} / \mathrm{F}_{\mathrm{M}}\right.$, see below) was compared daily between treatment and control groups of the same phylotype. When one-way ANOVA analysis for a particular T group showed a significant reduction in $\mathrm{F}_{\mathrm{V}} / \mathrm{F}_{\mathrm{M}}$ compared to its corresponding $\mathrm{C}$ group for two consecutive days, the temperature was recorded and determined to be the limit of that phylotype's thermotolerance under the experimental conditions. The T group was then returned to control conditions and monitored as before, and when daily $F_{v} / F_{M}$ measurements no longer showed a significant difference between the groups, the $T$ group was considered to have 'recovered'. In this manner the five phylotypes were assessed according to their thermotolerance, ability to recover from thermal stress (as measured by maximum quantum yield) and overall stability under the experimental conditions.

Measurement of $\mathrm{F}_{\mathrm{V}} / \mathrm{F}_{\mathrm{M}}$ was achieved through in vivo chlorophyll fluorescence measurements using an underwater pulse amplitude modulated fluorometer (PAM) (Underwater Fluorometer Diving PAM, Heinz Walz GmbH, Germany). Each replicate was dark adapted for 10 min to allow for relaxation of NPQ parameters, then individually illuminated via a low intensity measuring light (ML) $(0.15 \mu \mathrm{mol}$ photons $\left.\mathrm{m}^{-2} \mathrm{~s}^{-1}, 0.6 \mathrm{kHz}, 475 \mathrm{~nm}\right)$ provided by the fibre-optic light-sensor/emitter of the PAM fluorometer. By holding the sensor/emitter in direct contact with the bottom of the sample jar (where sufficient algal culture had accumulated), the measuring light excited the chlorophyll in the algae and detected the auto-fluorescence given off as the chlorophyll relaxed. The unitless fluorescence value 
obtained by the measuring light is termed "minimum fluorescence" $\left(\mathrm{F}_{0}\right)$ and represents the fraction of the total pool of reaction centres (RC's) in the sample that are currently closed (that is, RCs which are unavailable for photochemical reaction due to either having a reduced electron acceptor pool, being currently involved in nonphotochemical quenching or otherwise photoinactivated ie, due to physical damage). Following measurement of $\mathrm{F}_{0}$ the algae were subjected to a $800 \mathrm{~ms}$ saturating pulse (SP) of high intensity white light $\left(>1500 \mu \mathrm{mol}\right.$ photons $\left.\mathrm{m}^{-2} \mathrm{~s}^{-1}, 400-700 \mathrm{~nm}\right)$ with the subsequent peak in fluorescence termed "maximum fluorescence" or $F_{M}$. $F_{M}$ represents the fluorescence emitted when the total pool of reaction centres in the sample are closed due to complete reduction of the electron acceptor pool (Qa) downstream of PSII. Maximum quantum yield is then calculated as $F_{V} / F_{M}$, where $F_{V}=F_{M}-$ $\mathrm{F}_{0}$, or the difference between the pool of photosynthetically active and inactive RCs.

Measurements of $\mathrm{F}_{\mathrm{V}} / \mathrm{F}_{\mathrm{M}}$ were performed each morning after $1 \mathrm{~h}$ of light exposure, a timing regime chosen to avoid the effects of chlororespiration on quantum yield measurements. Chlororespiration occurs in the chloroplasts of Symbiodiniaceae during periods of darkness and involves the nonphotochemical reduction of intra-thylakoid plastoquinone (PQ) via a stromal pool of $\mathrm{NAD}(\mathrm{P}) \mathrm{H}$ (Jones \& Hoegh-Guldberg, 2001; Peltier \& Cournac, 2002). Fluorescence measurements performed on organisms with a pre-reduced PQ pool result in an underestimation of $\mathrm{F}_{\mathrm{M}}$, which subsequently leads to an artificially low $\mathrm{F}_{\mathrm{V}} / \mathrm{F}_{\mathrm{M}}$ calculation. Several minutes of light exposure after a prolonged period of darkness induces activation of the Calvin-Benson-Cycle (CBC), leading to re-oxidation of the PQ pool as NADP is reduced to NADPH at the terminal electron acceptor ferrodoxin-NADP reductase (FNR) in PSI. One hour of light exposure was chosen as sufficient time for PSI to process any pre-reduced PQ (Ross Hill \& Ralph, 2005), with the subsequent 10 min darkness allowing for complete re-oxidation of the electron acceptor pool, as excess reducing agents are consumed through the "dark reaction" of the CBC (Bassham et al., 1950). 


\subsection{Culture Experiment}

\subsubsection{Estimating required Biomass volume}

Before running the experiments, the minimum biomass of cells required for down-stream analysis was estimated. Preliminary protein extraction assays performed on cultures of Symbiodiniaceae phylotypes A4 and B2 using a "Qubit 2.0 Fluorometer" (Invitrogen), indicated that a final concentration of 2-4 million cells sample ${ }^{-1}$ was required to yield a lysate with a total protein concentration of $1-2 \mu \mathrm{g}^{-1} \mathrm{l}^{-1}$, a concentration considered sufficient for western blot analysis when loading 10-80 $\mu \mathrm{g}$ total protein per lane (S. C. Taylor \& Posch, 2014). The limiting factor when loading protein into SDS-PAGE is well volume, a variable dependent on the size of the 'comb' used when casting the gel. The well volume of a given $1.5 \mathrm{~mm}, 15$ well comb is $40 \mu \mathrm{l}(66 \mu \mathrm{l}$ for a $1.5 \mathrm{~mm}, 10$ well comb), meaning that a lysate with a total protein concentration as low as $0.5 \mu \mathrm{g} / \mu \mathrm{l}$ can (technically) be loaded to a final total protein volume of $20 \mu \mathrm{g}$ without overfilling the well. Preliminary western blot assays of PsbA standard dilutions against sample protein dilutions indicated that a sample volume of $10 \mu \mathrm{g}$ total protein was enough to accurately detect and quantify the target protein. Therefore, a sample yielding a lysate with a minimum total protein concentration of $0.5 \mu \mathrm{g} / \mu \mathrm{l}$ or $>\sim 100 \times 10^{4}$ cells sample $\mathrm{e}^{-1}$ was considered sufficient.

Cell counts of stock cultures (maintained at a constant temp of $25.5^{\circ} \mathrm{C}$ and $40 \mu \mathrm{mol}$ photons $\mathrm{m}^{-2} \mathrm{~s}^{-1}$ irradiance, on a $12 \mathrm{~h}: 12 \mathrm{~h}$ light : dark cycle) taken every 3-4 days over three weeks using a haemocytometer showed a concentration of $\sim 117 \times 10^{4}$ cells $\mathrm{ml}^{-1}( \pm 5.4 \mathrm{SE})$ for phylotype $\mathrm{B} 2$ and $\sim 49 \times 10^{4}$ cells $\mathrm{ml}^{-1}( \pm 4.3 \mathrm{SE})$ for phylotype A4. Assuming the above cell concentrations were the conditional equilibria for these phylotypes, and allowing for a maximum potential loss of $50 \%$ biomass during the experiment, a final sample volume of $5 \mathrm{ml}$ from each culture would be needed to yield a concentration of $\sim 294 \times 10^{4}$ and $\sim 123 \times 10^{4}$ cells sample ${ }^{-1}$ for B2 and A4 respectively. Therefore, an experiment with 32 replicates, and eight sampling points per replicate (See below), required a minimum volume of $1.3 \mathrm{~L}$ of culture with a minimum starting concentration of $\sim 40 \times 10^{4}$ cells ml ${ }^{-1}$. 


\subsubsection{Growth of Culture Biomass}

To achieve this volume of biomass, 20-30 ml of stock culture of either A4 or B2, (initial concentration of $\sim 30 \times 10^{4}$ and $\sim 75 \times 10^{4}$ cells $\mathrm{ml}^{-1}$ respectively) were inoculated into individual $1 \mathrm{~L}$ flasks ( $\mathrm{n}=5$ per phylotype) topped up to a total of $300 \mathrm{ml}$ with $\mathrm{f} / 2 \mathrm{ASW}$. The flasks were stoppered with gauze wrapped cotton wool and placed in a water bath set to $26^{\circ} \mathrm{C}$ under an irradiance of $\sim 100 \mu \mathrm{mol}$ photons $\mathrm{m}^{-2} \mathrm{~s}^{-1}$ on a $12 \mathrm{~h}: 12 \mathrm{~h}$ light : dark cycle. A $100 \mathrm{ml}$ aliquot of medium was removed weekly from each flask and replaced with $100 \mathrm{ml}$ of fresh $\mathrm{f} / 2$ enriched ASW to maintain exponential growth. Cell counts of four replicates from each were taken weekly using haemocytometer.

An antibiotic/antimycotic solution (A5955-100ML sigma Aldrich) was added to the media in all flasks at a final concentration of $1 \%$ to control cyanobacterial contamination and germanium dioxide $\left(\mathrm{GeO}_{2}\right)$ was added to all media to a concentration of $10 \mathrm{mg} \mathrm{L}^{-1}$ to inhibit diatom growth. While the physiological impacts this antibiotic/antimyotic solution on dinoflagellates is unkown, $\mathrm{GeO}_{2}$ has no demonstrable effect on non-siliceous microbes, (Markham \& Hagmeier, 1982; Shea \& Chopin, 2007)

\subsubsection{Experimental set up}

When sufficient Symbiodiniaceae biomass was available ( 7-8 weeks of growth time) the contents of the growth flasks were combined and a cell count taken, giving a pooled concentration of $\sim 120 \times 10^{4}$ and $\sim 145 \times 10^{4}$ cells $\mathrm{ml}^{-1}$ for A4 and B2 respectively. The pooled biomass of each phylotype was divided into 32 individual $250 \mathrm{ml}$ flasks by vacuum filtering either $40 \mathrm{ml}$ of A4 or $30 \mathrm{ml}$ of B2 through Q4 Whatman filter papers, then washing the collected biomass from each filter paper into $50 \mathrm{ml}$ of highnutrient $(\mathrm{HN})$ or low-nutrient $(\mathrm{LN})$ medium ( $\mathrm{n}=16$ for each treatment and phylotype). Cell density in the new flasks was approximately $90 \times 10^{4}$ cells $\mathrm{ml}^{-1}$ for each phylotype. This decrease in cell density in the experimental replicates relative to the pooled density was due to unavoidable losses incurred when washing culture biomass from the filter paper.

Experimental media consisted of ASW (salinity $34.5 \pm 1.5 \mathrm{ppt}$ ) made with ultrapure 'Milli-Q' water (MQ-ASW) and enriched with either full strength $\mathrm{f} / 2$ medium (HN) or a 1:1000 dilution of $\mathrm{f} / 2$ (LN). The final nutrient concentration for each medium was calculated as $472 \mu \mathrm{M}$ of total dissolved nitrogen 
(TDN) and $19 \mu \mathrm{M}$ of total dissolved phosphorous (TDP) for HN, and 4.6 $\mu \mathrm{M}$ TDN and $0.24 \mu \mathrm{M}$ TDP for LN, giving a similar N:P ratio of 21.35 and 18.83 respectively (Table. 2.1). Nutrient content of experimental media was determined by Lachat Flow Injection analysis and the Astoria 2 Analyzer System, performed on double replicate samples of MQ-ASW and MQ-ASW $+\mathrm{f} / 2$, by the NIWA Water Quality Laboratory, Hamilton, NZ. The final nutrient concentration for the LN medium was selected to mimic oligotrophic reef water as closely as possible (Libby \& Wheeler, 1997), while the HN content was chosen to prevent nutrient limitation occurring during the experiment.

Table 2.1: Nitrogen and phosphorous Content of Experimental Media. Reef water nutrient values taken from Libby \& Wheeler, (1997)

\begin{tabular}{|c|c|c|c|}
\hline Medium & TDN $(\boldsymbol{\mu M})$ & TDP $(\boldsymbol{\mu M})$ & $\mathbf{N}: \mathbf{P}$ \\
\hline High Nutrient (HN) & 472 & 19 & 21.35 \\
\hline Low Nutrient (LN) & 4.6 & 0.24 & 18.83 \\
\hline Reef Water & $\sim 3-9.5$ & $\sim 0.06-0.7$ & $\sim 13.6-50$ \\
\hline
\end{tabular}

\subsubsection{Thermal Treatment}

HN and LN were further divided into high and low temperature treatments (HT and LT), making four treatment groups per phylotype ( $\mathrm{n}=8$ for each). Four randomly selected flasks from each group of eight were then earmarked for future Lincomycin treatment (HN-LNT+L, HN-HT+L, etc.), but were otherwise treated identically to non-Lincomycin earmarked replicates. Lincomycin is an antibiotic which blocks protein synthesis in chloroplasts. Synthesis of D1 protein in -L samples will be compared with $+\mathrm{L}$ samples to determine the rate of chloroplastic protein synthesis under each treatment. LT replicates were kept in a water bath maintained at a constant $26^{\circ} \mathrm{C}( \pm 0.5)$ for the entire experiment. HT replicates were placed in an identical water bath where the temperature was gradually ramped up by $1{ }^{\circ} \mathrm{C}$ every two days, after an initial acclimation period of three days at $26^{\circ} \mathrm{C}$. Light exposure for all treatments was $\sim 115-125 \mu \mathrm{mol}$ photons $\mathrm{m}^{-2} \mathrm{~s}^{-1}$ on a $12 \mathrm{~h}: 12 \mathrm{~h}$ light:dark cycle, with temperature of both baths monitored every 15 min by HOBO pendants. The position of flasks was rotated daily to ensure even light exposure (Fig.2.1). 


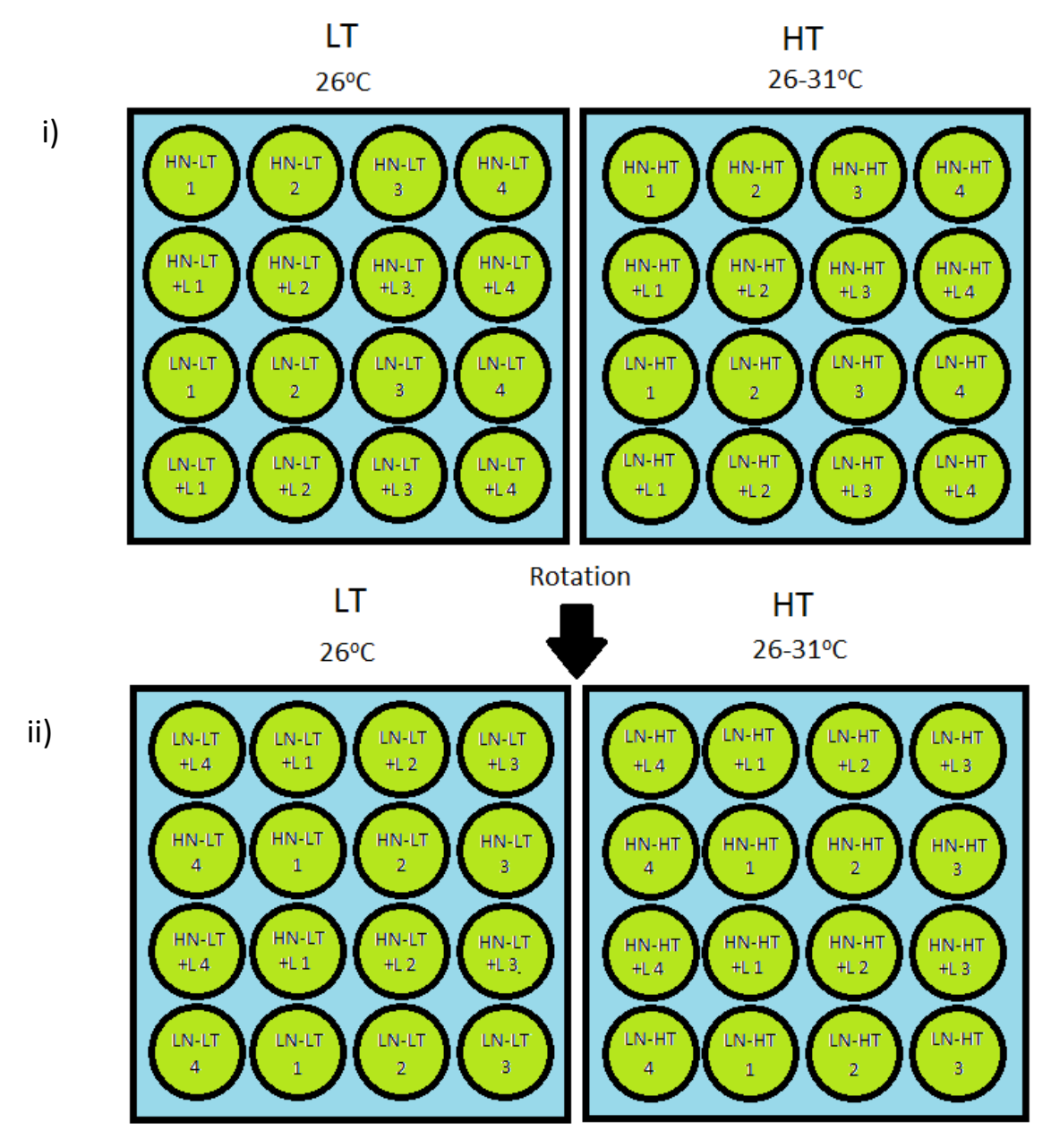

Fig 2.1 i) Visual representation of experimental set-up showing flasks of treatment groups (green circles) in their respective water-baths. li) New position of flasks after daily rotation. Flasks return to their original position every four days.

\subsubsection{Photosynthetic performance}

PAM measurements of maximum quantum yield were performed daily using a diving PAM as described previously. Briefly, all treatments were exposed to $1 \mathrm{~h}$ of light following the $12 \mathrm{~h}$ dark cycle, and then $\mathrm{F}_{\mathrm{v}} / \mathrm{F}_{\mathrm{m}}$ readings began after $10 \mathrm{~min}$ of dark acclimation. All measurements were performed in the dark by holding the PAM optical sensor against the base of the flask. Time taken to read all replicates was approximately 20-25 min, so readings were taken in random order to minimise any possible variation caused by differences in dark-exposure period between replicates. 
In addition, dark-light induction curves (DLIC's) were generated daily on selected replicates using a “Multiple Excitation Wavelength Chlorophyll Fluorescence Analyzer MULTI-COLOR-PAM' (MCPAM) (Walz, Germany). DLIC's chart the dynamic variation in effective quantum yield of PSII when a dark-adapted sample is exposed to actinic light over a short period of time (typically 5-10 min). Effective quantum yield is defined as $\left(F_{M}{ }^{\prime}-F\right) / F_{M}{ }^{\prime}=\Delta F / F_{M}{ }^{\prime}=Y(I I)$, where $F_{M}{ }^{\prime}$ is the effective maximum fluorescence measured by a saturation pulse applied to a light exposed sample. The difference between (dark-adapted) maximum fluorescence $\left(\mathrm{F}_{\mathrm{M}}\right)$ and (light exposed) effective maximum fluorescence $\left(\mathrm{F}_{\mathrm{M}}{ }^{\prime}\right)$ can be used to calculate non-photochemical quenching (NPQ) in a light exposed sample, where NPQ = $\left(\mathrm{F}_{\mathrm{M}^{-}}-\mathrm{F}_{\mathrm{M}}{ }^{\prime}\right) / \mathrm{F}_{\mathrm{M}}{ }^{\prime}=\mathrm{Y}(\mathrm{NPQ})$. A decrease in $\mathrm{F}_{\mathrm{M}}{ }^{\prime}$ can be translated as a decrease in the number of reaction centres (RCs) available for photosynthesis as de-epoxydation of xanthophyll pigments in the light harvesting complex (LHC) causes photonic excitation to be dissipated as heat ( $q \mathrm{E}$ ), and/or as LHCs undergo state transitions $(q \mathrm{~T})$ to effectively bypass PSII (Jahns \& Holzwarth, 2012; Ware et al., 2015). A third parameter termed $\mathrm{Y}(\mathrm{NO})$, or "constitutive losses", corresponds to the sum of non-regulated heat dissipation and/or fluorescence emission due to photoinhibited $\mathrm{RCs}$, and is calculated as $\mathrm{F} / \mathrm{F}_{\mathrm{M}}$ (Klughammer \& Schreiber, 2008). The real-time effective yield of these three parameters is unity, Y(II) $+\mathrm{Y}(\mathrm{NPQ})+\mathrm{Y}(\mathrm{NO})=1$. Comparison of multiple DLIC's over time provides information on a sample's ability to utilize NPQ under changing conditions, as well as highlight differences in stress response and photoinhibitory status between samples.

The MC-PAM is designed to perform pulse-amplitude fluorometry on dilute suspensions of photoautotrophs held in a $3 \mathrm{ml}$ quartz cuvette, and allows the user to manipulate a wide variety of parameters in real time via the UI software "PamWin". A key feature is the option to write a script file which automates certain processes, allowing samples to be exposed to identical stimuli over multiple days or weeks. By writing script files specific to the phylotypes under investigation, repetitive DLIC's were run using the following general protocol (Fig. 2.2)

A $1.2 \mathrm{ml}$ sample of culture was added to the $3 \mathrm{ml}$ cuvette, along with a "flea" stir-bar, and loaded into the MC-PAM optical unit. The optical unit was suspended above a stir-plate running at $\sim 25 \%$ speed to 
stop the culture settling out of suspension during the DLIC. The sample was allowed to dark-adapt for $10 \mathrm{~min}$ inside the optical unit. During this time a non-actinic measuring light $\left(<0.15 \mu\right.$ mol photons $\mathrm{m}^{-2}$ $\mathrm{s}^{-1}, 0.5 \mathrm{kHz}, 625 \mathrm{~nm}$ ) was applied to the sample to allow monitoring of $\mathrm{F}_{0}$ relaxation. After $10 \mathrm{~min}$ the sample was exposed to $15 \mathrm{~s}$ of weak, far-red (FR) light at $725 \mathrm{~nm}$. Light at this wavelength is not classed as photosynthetically active radiation (PAR, defined as light at wavelengths between $400-700 \mathrm{~nm}$ ) and is not absorbed by the LHC of PSII (which absorbs maximally at $680 \mathrm{~nm}$ ), and therefore does not induce electron flow through the ETC. Instead, the FR light is absorbed by PSI, a bias caused by conformational and structural differences between the LHCs of the two photosystems (Ishikita et al., 2006; Croce et al., 2007). This preferential excitation of PSI results in complete oxidation of the plastoquinone (PQ) pool downstream of PSII, as the incoming electron flow through PSI is utilized in tandem with any remaining reduced PQ to reduce $\mathrm{NADP}^{+}$to NADPH, effectively opening all available (non-photoinhibited) PSII reaction centres (Schreiber et al., 2012). Immediately following the 15 s FR exposure, the sample was subjected to an $800 \mathrm{~ms}$ saturating pulse (SP) ( 2500 $\mu \mathrm{mol}$ photons $\mathrm{m}^{-2} \mathrm{~s}^{-1}, 440-625 \mathrm{~nm}$ (White Light)) to determine maximum quantum yield, $\left(\mathrm{F}_{\mathrm{v}} / \mathrm{F}_{\mathrm{M}}\right)$. The sample was then exposed to a further $30 \mathrm{~s}$ darkness, to allow re-oxidation of $\mathrm{Q} a$ after the $\mathrm{F}_{\mathrm{v}} / \mathrm{F}_{\mathrm{m}}$ determining SP. The sample was then exposed to $6.5 \mathrm{~min}$ of continuous white actinic light (AL) $\left(162 \mu \mathrm{mol}\right.$ photons $\left.\mathrm{m}^{-2} \mathrm{~s}^{-1}\right)$, which is long enough for the sample to reach a steady state in terms of photochemical and non-photochemical yield of PSII (Y(II) and Y(NPQ) respectively) under the new light regime. The intensity of the AL was selected to be slightly higher than the experimental light intensity of $\sim 120 \mu \mathrm{mol}$ photons $\mathrm{m}^{-2} \mathrm{~s}^{-1}$, thus imposing a small increase in irradiative stress on the sample. This was to ensure that the response elicited by the AL included a measurable NPQ dynamic, while avoiding high-light induced photoinhibition in the sample. $15 \mathrm{~s}$ after the AL was switched on, the sample was exposed to a series of SPs at 1 min intervals $(\sim 2500 \mu \mathrm{mol}$ photons $\mathrm{m}^{-2} \mathrm{~s}^{-1}$, White Light) which allows for quenching analysis, or calculation of the change in $\mathrm{Y}$ (II), $\mathrm{Y}(\mathrm{NPQ})$ and $\mathrm{Y}(\mathrm{NO})$, as the sample moves toward steady state. After 6.5 min the AL was turned off and the sample allowed to relax back to a dark-adapted state. The interval between SPs was immediately increased to $2 \mathrm{~min}$, followed by a second increase to $3 \mathrm{~min}, 6 \mathrm{~min}$ later. After a further $12 \mathrm{~min}$, the interval was increased a final time to 5 min between SPs and the sample monitored for a further 15 min. The increase in SP interval over time is to ensure that the slow relaxation kinetics of the sample are not 
A.

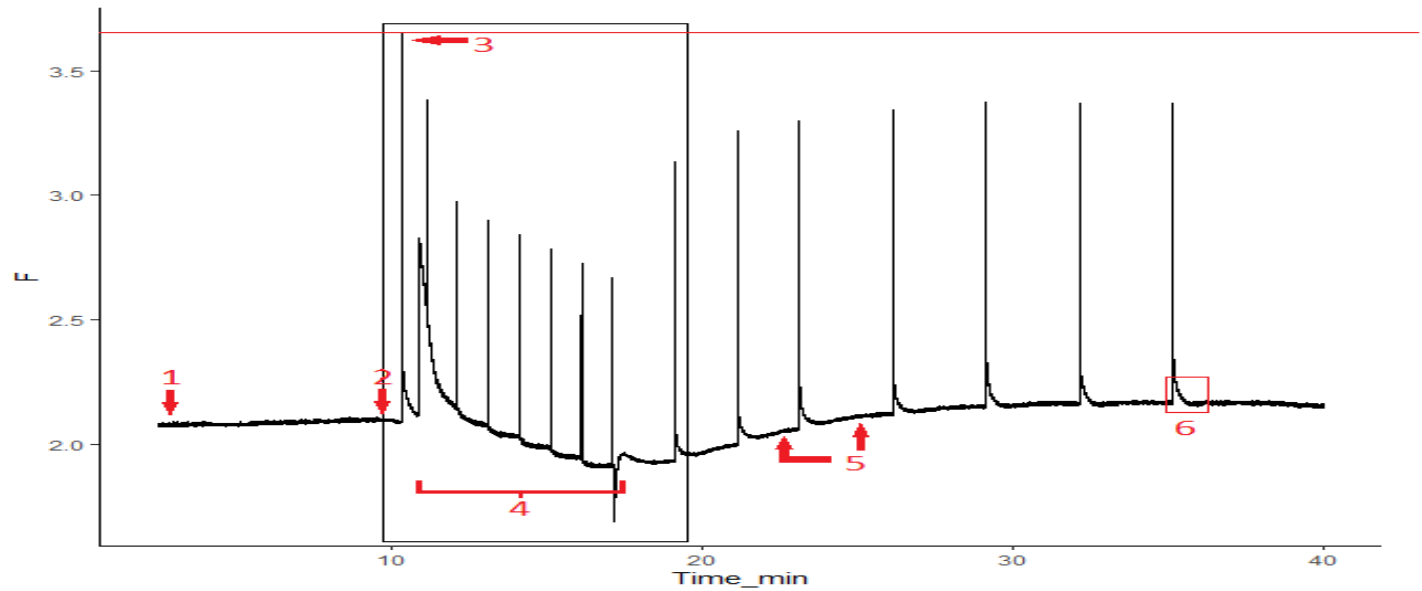

B.

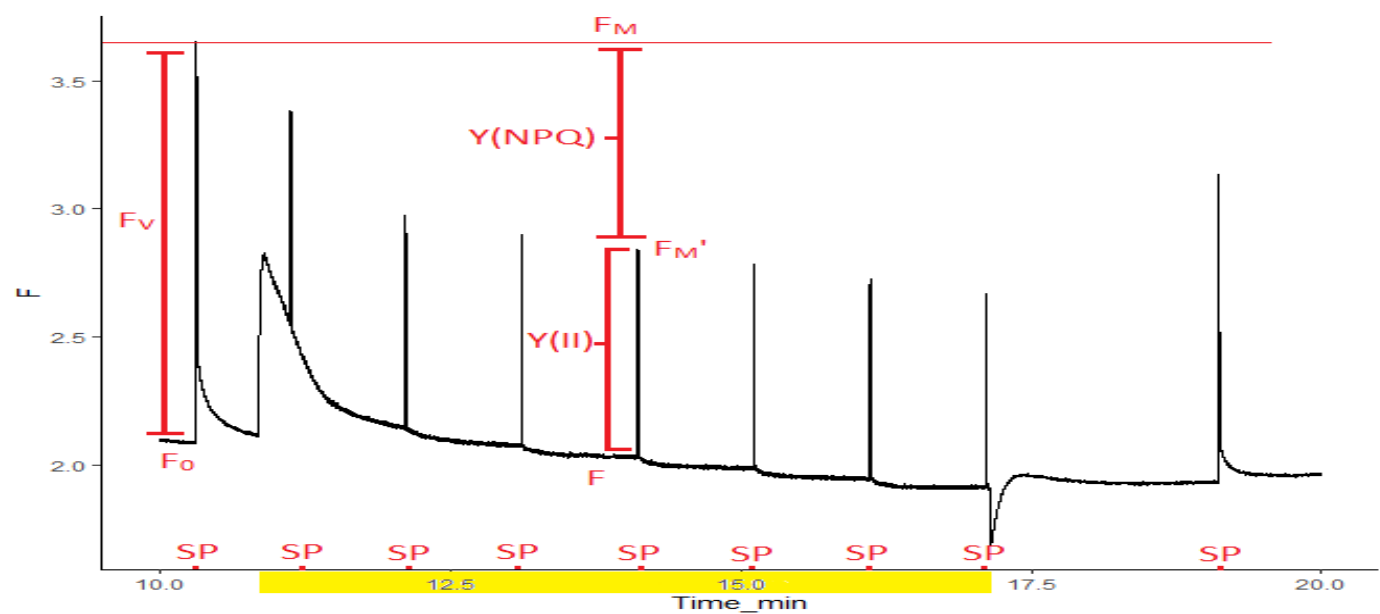

Fig.2.2_a) Fluorescence quenching analysis of dark-light induction curve (DLIC) charting sample fluorescence (F, unitless) against time in minutes, 1) Beginning of 10min dark adaptation period, 2) $15 \mathrm{sec}$ far-red light exposure, 3) Saturating pulse (SP) to determine maximum quantum yield, 4) Period of actinic light (AL) exposure, 5) Increase in interval between saturation pulses, 6) Non-immediate re-oxidation of Qa . 1.b) Magnified view of rectangular box section in a) $F_{V}=$ Variable Fluorescence, $F_{0}=$ Minimum Fluorescence, $F_{M}=$ Maximum Fluorescence, $F_{M}^{\prime}=$ Effective Maximum Fluorescence, $\mathrm{Y}(\mathrm{NPQ})=\left(\mathrm{F}_{\mathrm{M}}-\mathrm{F}_{\mathrm{M}^{\prime}}\right) / \mathrm{F}_{\mathrm{M}}, \mathrm{Y}(\mathrm{II})=\left(\mathrm{F}_{\mathrm{M}^{\prime}}-\mathrm{F}\right) / \mathrm{F}_{\mathrm{M}}, \mathrm{SP}=$ Saturation Pulse trigger, Yellow bar indicates period of $\mathrm{AL}$ exposure.

unduly influenced by the frequency of the SPs. Any exposure to high-light, including that of SPs, contributes protons to the lumenal proton pool and increases the intrathylakoid $\mathrm{pH}$ gradient. Unless these protons are readily consumed via ATP synthesis, the $\mathrm{pH}$ gradient will persist between SPs and $q E$ will be partially maintained (Papageorgiou \& Govindjee, 2004). This phenomenon is more pronounced in the dark, as slowing of the CBC due to reduced production of NADPH (a reaction which occurs only in the light, although see chlororespiration above) leads to a net reduction in available ADP and thus 
limited ATP synthesis in the thylakoid lumen (Schreiber, 2007).

\subsubsection{Sampling for protein extraction}

Mean $\mathrm{F}_{\mathrm{V}} / \mathrm{F}_{\mathrm{M}}$ of HT treatments were compared daily with the corresponding LT treatments using oneway ANOVA. When significant departure was recorded on two consecutive days between treatments, a sample for protein extraction was taken from all replicates on the second day. Samples were collected by pipetting $5 \mathrm{ml}$ of culture into a $5 \mathrm{ml}$ screw cap sample tube and were immediately frozen in liquid nitrogen before being transferred to a $-80^{\circ} \mathrm{C}$ Freezer. All replicates were left for a further $24 \mathrm{H}$ under the same conditions before a second sample was taken following the same method. Immediately after the second sampling, all $+\mathrm{L}$ replicates had Lincomycin added to a final concentration of $0.9 \mathrm{mM}$ (Hill et al., 2011; Hill \& Takahashi, 2014). The water bath containing HT treatments was then cooled to $26^{\circ} \mathrm{C}$ using freezer blocks ( $\sim 10 \mathrm{mins})$ and all treatments were exposed to 'recovery' light conditions of $\sim 20$ $\mu$ mole photons $\mathrm{m}^{-2} \mathrm{~s}^{-1}$ provided by full spectrum LED 'SolarOasis' overhead grow lights. Sampling was repeated 2, 8 and $24 \mathrm{~h}$ after exposure to the new temperature and light conditions (from here-on referred to as recovery conditions), while $\mathrm{F}_{\mathrm{V}} / \mathrm{F}_{\mathrm{M}}$ measurements were taken $2,4,6,8$ and $24 \mathrm{~h}$ after onset of recovery conditions.

\subsection{Western Blot Analysis}

\subsubsection{Protein Extraction}

Total protein extraction of culture samples for use in SDS-PAGE analysis was performed following guidelines Gallagher (2012) and Signore et al., (2017).

The previously collected $5 \mathrm{ml}$ samples were thawed, then transferred to $15 \mathrm{ml}$ falcon tubes and centrifuged for $5 \mathrm{~min}$ at $5000 \mathrm{rcf}$. The supernatant was discarded and the pellet re-suspended in $1 \mathrm{ml}$ of MQ $\mathrm{H}_{2} \mathrm{O}$ and vortexed briefly to wash off excess salts, before being transferred to a $2.5 \mathrm{ml}$ centrifuge tube. The sample was again centrifuged for $5 \mathrm{~min}$ at $5000 \mathrm{rcf}$ and the supernatant discarded. The pellet was re-suspended in $500 \mu \mathrm{l}$ of ice-cold lysis buffer (100 mM Tris, $100 \mathrm{mM} \mathrm{NaCl}, 10 \mathrm{mM}$ EDTA + a final concentration of $5 \%$ sodium-dodecyl-sulfate (SDS)) and immediately heated to $85^{\circ} \mathrm{C}$ for $10 \mathrm{~min}$ 
to denature any endogenous proteinases. All samples were kept on ice up to this point, except for when in the centrifuge, which was cooled to $4^{\circ} \mathrm{C}$.

After heating, samples were returned to ice and subjected to $20 \times 2 \mathrm{~s}$ pulses of $4 \mathrm{kHz}$ ultrasonication with $2 \mathrm{sec}$ intervals using a tapered $3 \mathrm{~mm}$ microtip (Vibra-Cell, Sonics). A final concentration of 5\% $\beta$-mercaptoethanol (2-ME) was added to each tube and the samples once more heated to $85^{\circ} \mathrm{C}$ for 15 $\min$. After heating, the samples were centrifuged a final time at 20,000 $\mathrm{rcf}$ for $5 \mathrm{~min}$ and the supernatant (now the protein lysate) transferred to a new $2.5 \mathrm{ml}$ tube and the pellet discarded. Protein lysates were kept in a $-80^{\circ} \mathrm{C}$ freezer until further analysis.

The high SDS concentration in the lysate precluded the use of standard spectroscopic assays such as Bradford or Lowry, therefore total protein quantitation was performed using a Qubit fluorometer, as per the manufacturer's instructions.

\subsubsection{SDS-PAGE Electrophoresis}

\subsubsection{Gel Casting}

The polyacrylamide gel through which the proteins are electrophoretically separated consists of two parts, a low density "stacking gel" which rests on top of a high density "resolving gel". Protein samples are loaded into the stacking gel and an electric current is applied, with the cathode (-) above the gels and the anode (+) below. The negatively charged proteins then migrate vertically downward from the stacking gel and through the resolving gel towards the anode.

To make the resolving gel $12 \mathrm{ml}$ of $30 \%$ acrylamide $/ 0.6 \%$ bisacrylamide $/ \mathrm{H}_{2} \mathrm{O}$ was added to $7.5 \mathrm{ml}$ of

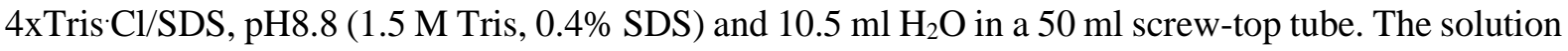
was allowed to degas for 10 min before adding $100 \mu \mathrm{l}$ of $10 \%$ (w/v) ammonium persulfate and $20 \mu \mathrm{l}$ of tetramethylethylenediamine (TEMED) to initiate polymerization. The final solution was gently mixed, then immediately pipetted into a $14 \mathrm{~cm}$ x $8 \mathrm{~cm}$ x $1 \mathrm{~mm}$ glass gel-casting sandwich (Mini-PROTEAN, BioRad), consisting of two glass plates clamped together in a casting stand. A $5 \mathrm{~mm}$ layer of 2-propanol alcohol was pipetted on top of the gel to exclude oxygen (which inhibits polymerization) and to allow a flat interface to form on the gel. The gel was allowed to polymerize for $\sim 45$ mins before the 2-propanol 
was poured off and the gel rinsed 3-4x with Tris Cl/SDS, pH8.8.

The stacking gel was made by mixing $1.3 \mathrm{ml}$ of $30 \%$ acrylamide/ $0.8 \%$ bisacrylamide, $2.5 \mathrm{ml}$ of $4 \mathrm{x}$ Tris Cl/SDS, pH6.8 (0.5 M Tris, 0.4\% SDS) and $6.1 \mathrm{ml} \mathrm{H} \mathrm{H}_{2} \mathrm{O}$ in a $15 \mathrm{ml}$ screw-top tube. The solution was allowed to degas for $10 \mathrm{~min}$ (by placing the tube inside a low-pressure vacuum flask) before adding $50 \mu 1$ of $10 \%$ (w/v) ammonium persulfate and $10 \mu 1$ TEMED. The solution was gently mixed and then pipetted on top of the polymerized resolving gel, filling the casting sandwich to $\sim 5 \mathrm{~mm}$ from the top. A Teflon loading-well comb inserted into the top of the stacking gel produced cavities, or 'loading-wells', into which the protein samples will be loaded (with either 10 or 15 teeth, depending on the required volume of protein sample) and the stacking gel allowed to polymerize for $\sim 45 \mathrm{~min}$ at room temperature. After polymerization, the comb was removed and the loading wells rinsed with SDS-electrophoresis buffer (25 mM Tris, $192 \mathrm{mM}$ Glycine, 0.5\% SDS). The polymerized gels, still in their casting sandwiches, were then introduced into an electrode cell and placed into an electrophoresis chamber filled with SDS-electrophoresis buffer.

The resolving gel acrylamide concentration was chosen to allow for optimal resolution of a target protein with a molecular weight (MW) of 15-35 kilodaltons (kDa) (D1 protein has a MW of 38.9kDa, but migrates with an apparent MW of between 30-35kDa). The stacking gel acrylamide concentration is standardised and independent of the target protein, as its function, achieved through a combination of low porosity and $\mathrm{pH}$, is to concentrate the proteins into a thin stack before they enter the resolving gel.

\subsubsection{Sample Preparation}

While the gels were polymerizing, the protein lysates were diluted in either $2 \times$ or $6 \times$ concentration SDS sample buffer (SB), depending on the required total protein concentration to be run $(6 \times \mathrm{SB}=7$ $\mathrm{ml} 4 \mathrm{x}$ Tris $\mathrm{Cl} / \mathrm{SDS}$ (pH6.8), $3 \mathrm{ml}$ glycerol, $1 \mathrm{~g}$ SDS, $1.2 \mathrm{mg}$ bromophenol blue). The SDS in the sample buffer binds with the proteins, keeping them in a denatured state and lending them a net negative ionic charge, essential for electrophoretic interaction (Bhuyan, 2010), while the glycerol adds density to the 
lysate for easier handling and the bromophenol blue makes the sample visible within the gel. A sample of each culture lysate was added to a $250 \mu$ l centrifuge tube and diluted to either 10, 15, 20, 25 or 30 $\mu \mathrm{g}$ of total protein concentration per sample load, depending on the target protein concentration of the individual lysate, using $2 \times \mathrm{SB}$ at a dilution of 1:1. For lysates with very low total protein and/or target protein concentrations, 6 x SB was used at a dilution of 1:5, in order to keep the final required volume of sample below the allowable volume of the loading well. Lastly, a sample dependent volume of 2ME was added to a final concentration of 5\% and the tubes spun for $\sim 10-15 \mathrm{sec}$ in a micro-centrifuge, before being placed in a thermal block and heated to $85^{\circ} \mathrm{C}$ for $5 \mathrm{~min}$. This final addition of 2-ME was a redundancy measure to ensure excess reducing agent was available to reduce any remaining disulphide bonds within the proteins.

\subsubsection{D1 Protein Standard}

In order to accurately quantify target protein concentration in a sample, each gel was required to contain a standard curve consisting of a series of three, two-fold dilutions of a known concentration of D1 protein standard (Agrisera, AS01 016S). For accurate target protein quantitation, the range of the D1 protein standard (D1-STD) must encompass the range of target protein concentrations present in all samples included in a gel, and it was therefore necessary to group and run samples of similar target protein concentrations together to ensure they all fit within the same D1-STD range. Typically, this was achieved by loading together protein samples representing the four biological replicates of a single sample point, as their target protein concentrations would tend to be in a similar range, along with four biological replicates from a second sample point that had a range of target protein concentrations comparable to the first. While this method of grouping samples excluded the level of randomisation normally recommended in such analyses (Aldridge et al., 2008), it was necessary in order to allow for accurate downstream determination of target concentration. Maintaining short ranges within the protein standards allowed for higher accuracy in both linear regression analysis and optical density measurements, but at the cost of randomisation in sample loading (Fig. 2.3). The lowest range of D1STD used was $0.003125-0.0125$ pmol, and the highest $0.025-0.1$ pmol. 

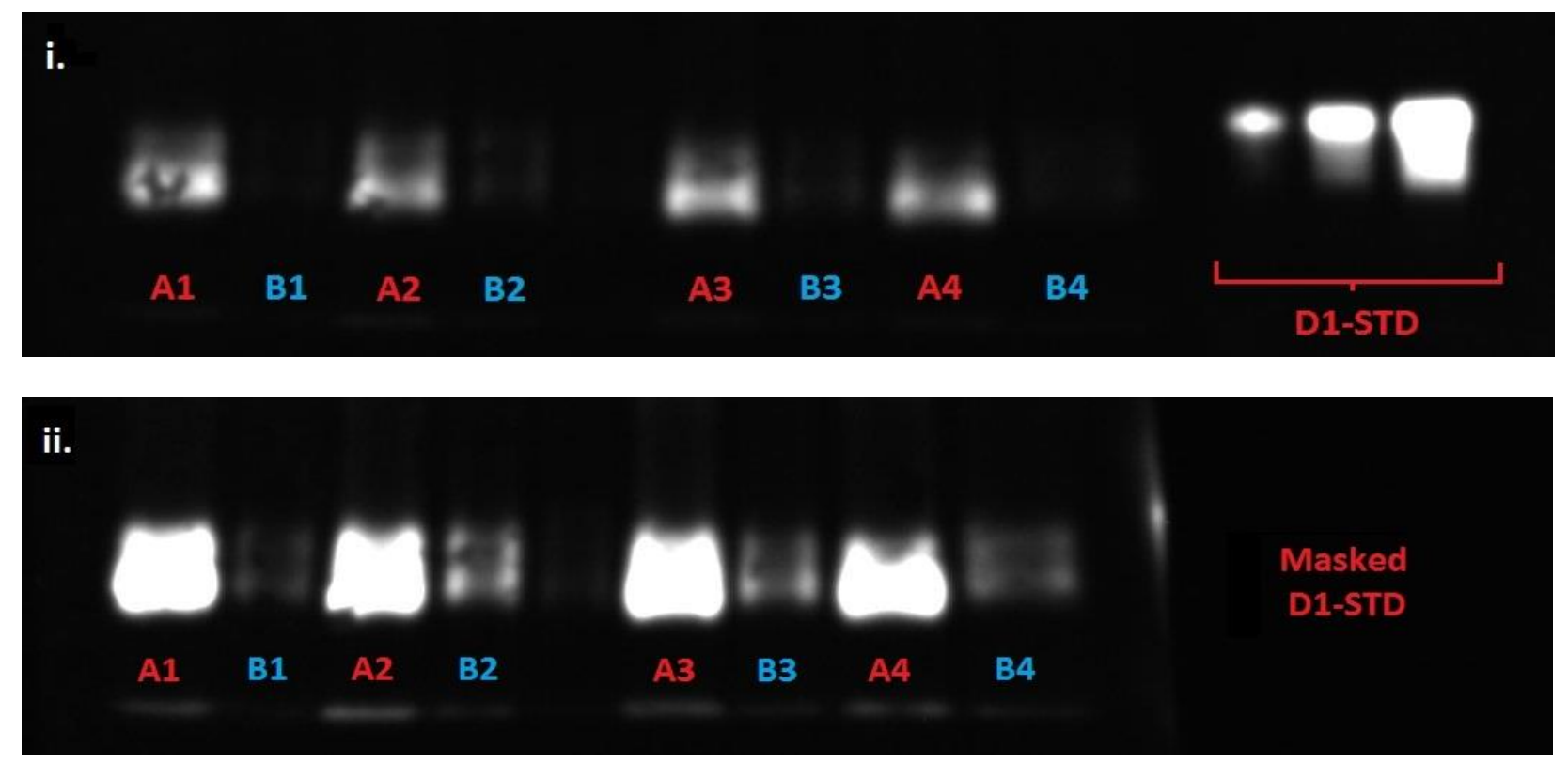

Fig. 2.3 Example of issues arising when imaging high protein samples alongside low. Both images are of the same membrane, blotted with protein samples from two separate time points (A and B) with four biological replicates from each (A1-4 and B1-4) along with three two-fold dilutions of D1 protein standard (D1-STD). i) Samples A1-4 fit within the range of the D1-STD and can be quantified, but samples B1-4 are under-exposed and not quantifiable. ii) The area of the D1-STD was covered with tin-foil and the membrane re-imaged on a longer exposure. Now samples B1-4 are detectable, but samples A1-4 are over exposed (saturated) and not able to be quantified accurately.

\subsubsection{Total Protein Standard}

A second standard was included in each gel for the purpose of normalizing the sample's target protein concentration against its total protein concentration. This total protein standard (TP-STD) was necessary to ensure that apparent variations in target protein concentration between samples weren't artefacts of experimental irregularities, such as actual differences in the amount of sample loaded or uneven protein transfer from the gel to the membrane(Aldridge et al., 2008; Moritz, 2017; PillaiKastoori et al., 2020). The TP-STD represents the average total protein concentration of all lysates in a particular experiment, and was made by pooling $10 \mu \mathrm{l}$ from each lysate from all time points across all treatment groups. Thus, $10 \mu 1$ x 32 replicates x 5 time-points gave a total pooled lysate volume of 1.6 $\mathrm{ml}$ for each phylotype. The total protein concentration of the pooled lysate was determined as the mean of triplicate readings from five sample replicates as measured by a Qubit fluorometer. Total protein loads for each group of samples reflected the optimal load necessary to detect the target protein within 
a quantitative linear D1-STD range. Requisite total protein loads were determined via preliminary WB assays, where lysates of unknown target protein concentration were run against varying D1-STDs to find the optimal total protein volume. Assays seeking to affirm linearity of total protein concentration against detectable signal were run for both phylotypes. The TP-STD and experimental samples for a single gel were all diluted to the same total protein concentration before loading onto the gel.

\subsubsection{Loading of gels and electrophoresis}

Each completed 15 well gel was loaded with a total of eight protein samples (representing four biological replicates from each of two time points), one total protein standard, three D1 protein standard dilutions, and a protein ladder (a sample of stained protein fragments of predetermined length used for identifying the apparent molecular weight of sample proteins (PageRuler, Thermoscientific)). The first and final wells of the gel (1 and 15) were loaded with a 'blank', or sample buffer with no protein content, with the same volume as the protein samples. This was to avoid 'edging' where samples loaded in end lanes spread towards the edge of the gel and become distorted or lost. Wells two to five and seven to ten were loaded with alternating samples from each of the two time points (see fig 2.3). The sixth well was loaded with the TP-STD, a position chosen to best represent protein losses due to uneven protein transfer to the membrane (See 'Normalization'). Well 11 was loaded with $2 \mu 1$ of protein ladder and the D1-STD was loaded in wells 12-14, at volumes of 5-10 $\mu 1$, depending on the required dilution.

Electrophoresis was performed under a constant 120 volts for 120 minutes using a Bio-Rad PowerPac HC (BioRad) high current power supply at room temperature. This was enough time for the smallest proteins in the sample to migrate to $\sim 1.5 \mathrm{~cm}$ of the bottom of the gel, as judged by monitoring of the blue dye in the sample buffer.

\subsubsection{Protein Transfer}

After electrophoretic separation of the proteins, the power supply was turned off and the gel casting sandwich removed from the electrophoresis tank. A piece of polyvinylidene fluoride (PVDF) membrane (Immobilon-FL, pore-size $0.45 \mu \mathrm{m}$ ), the membrane onto which the proteins were to be transferred, was 
cut to the same dimensions as each gel and soaked in $100 \%$ methanol (MeOH) for 10-15 sec. This step is necessary to activate the membrane, as PVDF is extremely hydrophobic and will resist the flow of aqueous buffer components if not fully 'hydrated' with alcohol before use (Kurien \& Scofield, 2009). The membranes were then equilibrated in Towbin's transfer buffer (25 mM Tris, $192 \mathrm{mM}$ glycine $20 \%$ methanol / 80\% $\mathrm{dH}_{2} \mathrm{O}(\mathrm{vol} / \mathrm{vol})$ (Towbin et al., 1979)) for $\sim 10$ min on a rocker plate. Gel-membrane sandwiches were then built for each gel, starting with a sponge, followed by buffer-soaked filter paper, followed by the gel. The equilibrated PVDF membrane was laid on top of the gel, with any air bubbles rolled out using a plastic roller and a second piece of buffer-soaked filter paper placed on top of the membrane with a final plastic sponge placed on top. The entire sandwich was locked into a transfer cassette loaded into an electrode fitted blotting module which was then introduced into a transfer tank filled with Towbin's transfer buffer,

In order to optimise protein transfer, blotting was routinely performed at a constant 30 volts over $16 \mathrm{~h}$ (overnight). This allowed ample time for migration of all proteins out of the gel. To avoid joule heating of the buffer during the $16 \mathrm{~h}$ transfer, a freezer block was placed inside the transfer tank next to the blotting module and the tank packed in a polystyrene box full of crushed ice. The transfer was then run in a cold room at $4^{\circ} \mathrm{C}$.

\subsubsection{Immunodetection}

\subsubsection{Blocking}

At the conclusion of protein transfer, the sandwiches were removed from the blotting module and the membranes immediately placed into separate plastic air-tight containers with $\sim 10 \mathrm{ml}$ blocking agent (5\% low fat milk powder + Tris buffered saline (TBS, $20 \mathrm{mM}$ Tris, $150 \mathrm{mM} \mathrm{NaCl}, \mathrm{pH} \mathrm{7.6)}$ ) and incubated for $60 \mathrm{~min}$ at room temperature (RT), with agitation provided by an orbital shaker. This is essential for the immunodetection process, as it ensures that the antibodies used to detect the target protein will only bind to the target protein and not to non-specific parts of the membrane. Following incubation, the blocking agent was discarded and the membranes washed once for five minutes with Tris buffered saline + Tween 20 (TBST, TBS + 0.1\% Tween 20), at RT with agitation.. 


\subsubsection{Antibody incubation}

The primary antibody used in these experiments was a polyclonal antibody raised against a C-terminal epitope of the D1 protein (Agrisera, AS05 084) with a rabbit host. This is referred to as the primary antibody $\left(1^{\circ} \mathrm{AB}\right)$ in the context of immunodetection as it binds directly to an antigen on the target protein. The secondary antibody $\left(2^{\circ} \mathrm{AB}\right.$, Goat anti-Rabbit IgG, (Agrisera, AS10 668)) was a polyclonal antibody raised against rabbit antibodies introduced into a goat (family Bovidae). The resulting goatanti-rabbit antibodies were conjugated with horse radish peroxidase (HRP), an enzyme found in the roots of horseradish (Armoracia rusticana) which catalyzes the oxidation of luminol to 3-aminopthalate in the presence of hydrogen peroxide $\left(\mathrm{H}_{2} \mathrm{O}_{2}\right)$ to emit low intensity light via chemiluminescence. This reaction is enhanced $\sim 1,000$ fold when in the presence of phenolic enhancers (enhanced chemiluminescence, ECL), to produce a detectable and quantifiable light source, the intensity of which is directly correlated with the amount of HRP present (Sanchez et al., 1995; Haan \& Behrmann, 2007). The secondary antibody $\left(2^{\circ} \mathrm{AB}\right)$ binds to an antigen on the $1^{\circ} \mathrm{AB}$ rather than to the target protein, meaning non-specific binding (binding to any molecule other than the intended target), by either $1^{\circ} \mathrm{AB}$ or $2^{\circ} \mathrm{AB}$ can confound quantification of target proteins.

The membranes were incubated in $1^{\circ} \mathrm{AB}$ diluted 1:40,000 in $5 \mathrm{ml} \mathrm{TBST}$ for $60 \mathrm{~min}$ at RT with agitation. The antibody/TBST solution was then discarded and the membranes rinsed briefly in TBST, before being washed three times for five minutes per wash in fresh TBST at RT with agitation (15 min altogether), discarding the TBST between washes to ensure no unbound or non-specifically bound $1^{\circ} \mathrm{AB}$ remained on the membrane. Membranes were then incubated in $2^{\circ} \mathrm{AB}$ diluted 1:40,000 in $5 \mathrm{ml}$ TBST $+1 \%$ non-fat milk, for $60 \mathrm{~min}$ at RT with agitation. The non-fat milk was added to limit non-specific binding of the $2^{\circ} \mathrm{AB}$ in areas of the membrane which may have opened up during the previous washes. Membranes were washed again as above, followed by a final 5 min wash with TBS to remove any residual detergent (Tween 20). 


\subsubsection{Target Protein Imaging}

Membranes were incubated for $5 \mathrm{~min}$ at RT with agitation in a non-commercial ECL solution (100 mM Tris/ $\mathrm{HCl} \mathrm{pH}$ 8.8, $1.25 \mathrm{mM}$ luminol, $2 \mathrm{mM}$ 4-iodophenylboronic acid (4-IPBA), $5.3 \mathrm{mM} \mathrm{H}_{2} \mathrm{O}_{2}$ ) made immediately before each use from stock solutions of $100 \mathrm{mM}$ 4-IPBA/dimethyl sulfoxide (DMSO) and $250 \mathrm{mM}$ luminol/DMSO. Membranes were then immediately transferred to a charge-coupled device (CCD) imager (Amersham Imager 600, Amersham) for chemiluminescence detection. Exposure times were typically set to automatic, with exposures lasting $\sim 1-15 \mathrm{~min}$, depending on the quantity of target protein and/or D1-STD present on the membrane. An option for incremental exposure allowed the capturing of cumulative exposures (every $2-3$ min over a period of $15-20 \mathrm{mins}$ ), and was used when imaging membranes with high background or particularly low protein loads, to return a range of images with varied exposure times. The light emitting oxidation reaction of luminol peaks within $10 \mathrm{~min}$ of the addition of ECL, with a half-life of $\sim 1 \mathrm{~h}$ (Young, 2009), so membranes were incubated with ECL immediately before detection to maximise optimal imaging time.

\subsubsection{Total Protein Staining}

In order to normalize target protein concentration, it was necessary to visualize and compare total protein concentration across all samples, and correct for deviations in total protein abundance between sample loads (Taylor et al., 2013). This was achieved through staining of post-immunodetection membranes with either Coomassie brilliant blue R-250 (Sigma-Aldrich, B7920) or Fast Green FCF (Sigma-Aldrich, F72652). Early staining of membranes carrying protein samples of phylotype B2 found that CBB gave relatively poor results in terms of band definition, and therefore the more sensitive FG dye was used for these membranes.

After imaging of target proteins, membranes were washed for $5 \mathrm{~min}$ in TBST, followed by $5 \mathrm{~min}$ in TBS to remove detergent residue. Membranes were rehydrated for $15-20 \mathrm{sec}$ in $\mathrm{MeOH}$, followed by 5 min equilibration in de-staining solution $(30 \% \mathrm{MeOH}, 7 \%$ Acetic acid v/v). They were then stained for 10 min in CBB stain (10\% Acetic acid, 40\% MeOH, 50\% $\mathrm{H}_{2} \mathrm{O}, 0025 \% \mathrm{CBB}$ ) or FG stain (7\% Acetic acid, $30 \% \mathrm{MeOH}, 63 \% \mathrm{H}_{2} \mathrm{O}, 0.001 \% \mathrm{FG}$ ), followed by 10 min of de-staining (solution as above). 
Membranes were then rinsed briefly in $\mathrm{H}_{2} \mathrm{O}(10-15 \mathrm{sec})$, followed by a final wash in $\mathrm{H}_{2} \mathrm{O}$ for 10 mins. MQ water was used for all steps, and washing, staining and de-staining was performed at RT with agitation provided by an orbital shaker. After the final wash, membranes were allowed to air-dry overnight.

Imaging of total protein was performed using a FLA-5100 Fluor Imager (Fujifilm) laser scanner with the following settings: $532 \mathrm{~nm}$ laser(CBB), long-pass red filter (LPR, $>625 \mathrm{~nm}$ ), $25 \mu \mathrm{m}$ pixel size, photomultiplier tube (PMT) set to 500.

\subsection{Image Analysis}

All image analyses were performed using 'ImageJ' image processing software (https://imagej.nih.gov), with image files imported in .tif file format.

\subsubsection{Target Protein Optical Densitometry}

Digital images of chemiluminescent signals were used to determine the optical density (OD) of target proteins via measurements of raw integrated density (RawIntDen) (Fig. 2.4). RawIntDen is the sum of the values of pixels in a region of interest (ROI) of a digital image, with pixel values corresponding to the signal, or intensity of light, detected by the imaging sensor when exposed to the chemulinescent sample. A ROI was designated by placing a rectangular selection area over a target band (a band of pixels representing the target protein), and a measurement taken of the corresponding RawIntDen (as calculated by the ImageJ software). A second measurement, using the same sized ROI, was then taken immediately above the target band, and the RawIntDen of the second ROI subtracted from the first (Aldridge et al., 2008). The remainder of the two RawIntDens represents the final OD of the target band (target OD), minus any background signal caused by nonspecific binding of the antibodies as well as any signal inherent to the membrane itself each image (Gassmann et al., 2009). The RawIntDen capture method was applied to all nine target bands (eight sample bands and one TP-STD band), as well as the three D1-STD bands for each membrane. The calculated optical density of the three D1-STD bands was 
then used to build a linear regression model from which the protein concentration of each target band within a membrane was determined.

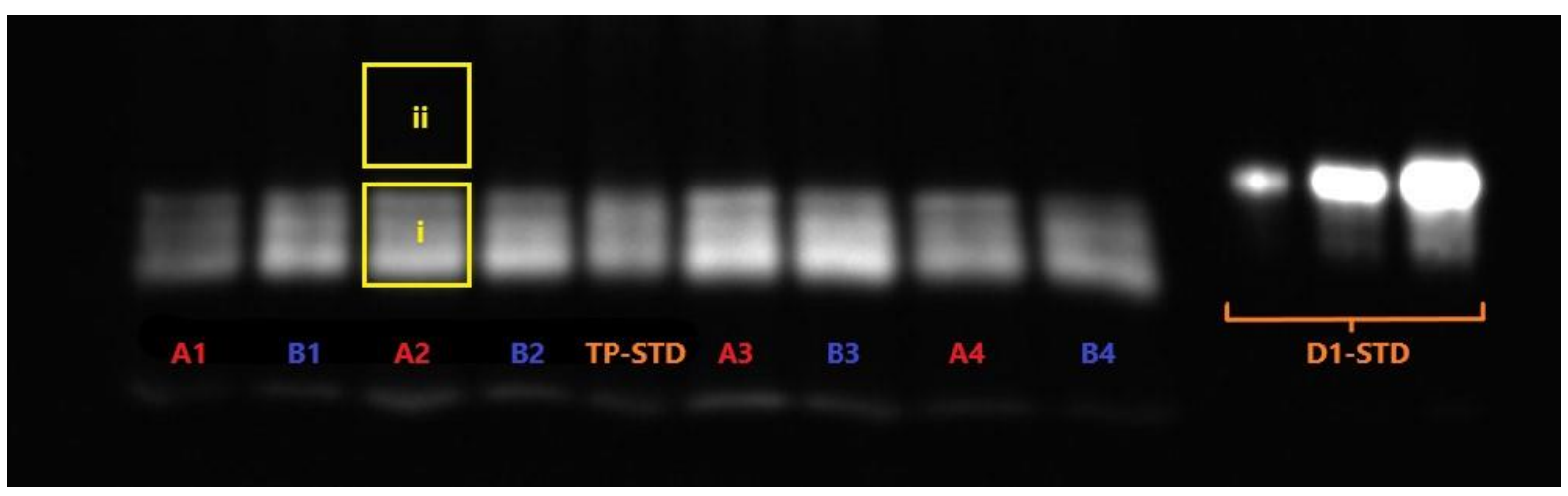

Fig. 2.4 Example of two regions of interest (ROls, yellow boxes) used to calculate the optical density of a target band. ROI-i) Raw integrated density (RawlntDen) of target band measured as the sum of the value of all pixels in the ROI. ROI-ii) Measurement of RawIntDen of background signal. Subtracting the RawIntDen value of ROI-ii from ROI-i gives the final optical density of target band A2. A1-4=Sample group A 1-4; B1-4= Sample group B 1-4; TP-STD=Total protein standard; D1-STD=D1 protein standard curve.

\subsubsection{Total Protein Optical Densitometry}

Digital images of fluorescence emission originating from protein bound dyes (CBB or FG) were analysed to determine the optical density (OD) of total protein in each lane, using a variation of a technique proposed by Aldridge et al., (2008). In order to accurately compare OD as a measure of protein load between samples, it is necessary to ensure that the ROI's being compared represent the same amount of protein, minus any background signal. The above method of subtracting the RawIntDen of $\mathrm{ROI}_{\mathrm{ii}}$ from an equally sized $\mathrm{ROI}_{\mathrm{i}}$ is not feasible in this case, as there is no equally sized $\mathrm{ROI}_{\mathrm{ii}}$ in the immediate vicinity of $\mathrm{ROI}_{\mathrm{i}}$ which would accurately represent the background signal (Fig. 5). Aldridge et al., (2008) proposed using a thin slice $\left(\mathrm{ROI}_{\mathrm{i}}\right)$ running vertically down the centre of each lane, and then subtracting the same sized slice $\left(\mathrm{ROI}_{\mathrm{ii}}\right)$ taken from between the lanes. However, it is common for lanes to spread out horizontally to varying degrees during electrophoresis, meaning a thin vertical slice of one lane may not cover the same amount of protein as the same sized slice of the lane next to it (Fig. 2.5a). Therefore, using RawIntDen as a measure of optical density in this case was deemed unsuitable, 
and it was necessary to instead compare the mean intensity (MI, mean grey value of all pixels) of different sized ROIs. By using the segmented selection tool, a ROI was created which encompassed the entire width and a defined length of each lane, as measured against the molecular weight marker, and a measurement of MI taken (Fig 2.5b). Two ROIs were created on either side of the lane, of equal length to the first ROI but thinner, so as to include only background signal (Fig. 2.5c). The mean of the MI's from the second two ROIs was then subtracted from the MI of the first, with the remainder representing the final mean OD of that segment of the lane, minus background signal. This was repeated for all lanes on a membrane and the final mean OD of each lane then used for total protein normalization

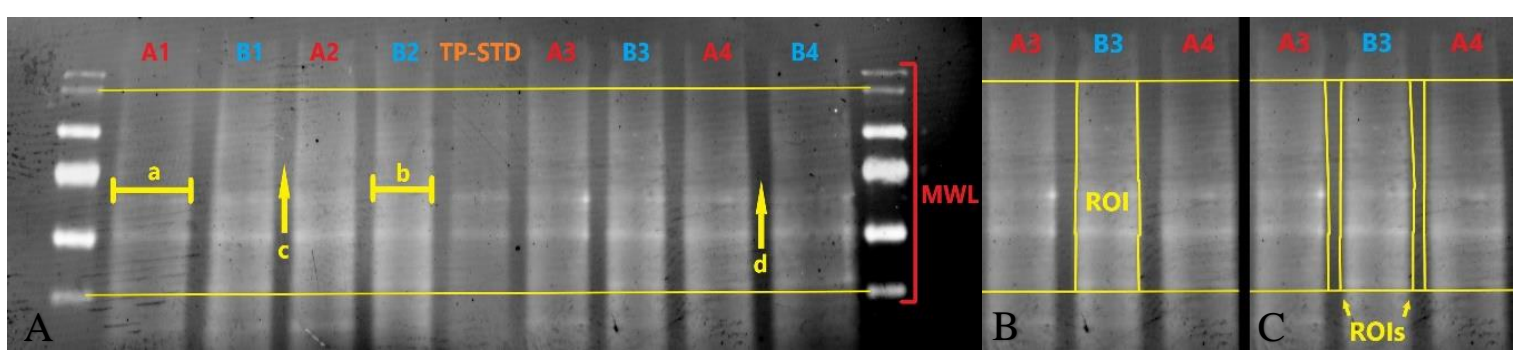

Fig. 2.5 Process of determining total protein optical density, A) Digital image of stained protein fluorescence with example of unequal lane width (a \& b) and uneven background signal (c \& d). B) Region of interest (ROI) drawn around protein sample B3 to measure mean intensity of fluorescence signal. Upper and lower limits of ROI standardized by molecular weight ladder (MWL) to ensure equal protein load measurement across lanes. C) Two ROIs drawn either side of protein sample B3 to measure mean background signal. This is subtracted from the ROI mean intensity in image B, giving mean total protein optical density of sample B3. Image shown contains two MWL's, one on each side of the sample lanes, later membranes contained only one MWL, to the right of the sample lanes. A1-A4, B1-B4=Protein samples A1-4 and B1-4 respectively, TP-STD=Total protein standard

\subsubsection{Normalization and Data Analysis}

The mean optical density (MOD) of each sample lane (obtained from the total protein fluorescence analysis) was normalized to the MOD of the TP-STD of its corresponding membrane, by dividing the sample MOD by the TP-STD MOD to give a total protein adjustment factor. The target OD for each lane (obtained from the target protein chemiluminescence analysis) was then divided by its corresponding total protein adjustment factor, to give a target OD adjusted to the total protein content 
of the sample and normalized against the TP-STD (Eaton et al., 2013; Degasperi et al., 2014; Janes, 2015).

A further normalization step was performed to account for variations in loading and transfer irregularities between membranes. To do this, apparent target protein concentrations of the TP-STDs were determined according to linear regression models built from the OD of their respective D1-STDs plotted against the known D1-STD concentrations. These values were then summed across all membranes and the apparent concentration of each TP-STD divided by the mean of the total, giving a target protein adjustment factor for each membrane. The adjusted target OD of each sample from the first normalization step was then divided by its corresponding membrane's target protein adjustment factor to give the final target OD for each lane. The final target ODs were then used to determine the apparent target protein concentration of each sample, as determined by their respective D1-STD linear regression models. The apparent target protein concentrations for each set of four biological replicates were then averaged, giving the mean D1 protein concentration for each treatment group at each time point. Only D1-STD regression models with $\mathrm{R}^{2}$ values $\geq 0.97$ were used to determine apparent protein concentrations. Membranes with curves failing to meet this value were rejected, and the blots re-run using a different range of D1-STD concentrations.

Entry and statistical analyses of OD data were performed using Microsoft Excel and RStudio.

\subsection{Aiptasia Experiment}

The model symbiotic anemone Aiptasia was inoculated with Symbiodiniaceae and subjected to fed or starved feeding regimes, followed by heat stress to determine the influence of Symbiodiniaceae phylotype on the heat tolerance of the symbiotic partnership. 


\subsubsection{Rearing of Experimental Organisms}

Experimental organisms were gathered from a clonal, lab reared population of the sea anemone Aiptasia (Aiptasia pallida; culture ID 'NZ1' of unknown Pacific origin) which had been experimentally 'bleached' by the addition of menthol to their surrounding seawater (Matthews et al., 2016), rendering them free of symbiotic algae (aposymbiotic). These aposymbiotic anemones were kept in $0.22 \mu \mathrm{m}$ filtered seawater at $26^{\circ} \mathrm{C}$ under constant darkness prior to commencement of the experiment.

A total of 960 aposymbiotic anemones were divided into $12 \times 300 \mathrm{ml}$ clear plastic jars (80 anemones per jar) containing $250 \mathrm{ml}$ of ASW made with Milli-Q purified $\mathrm{H}_{2} \mathrm{O}$ (MQ-ASW). This number of anemones was chosen to allow three replicates each consisting of three anemones to be harvested at each of five time points across the experiment, while also allowing for some mortality. The jars were earmarked for inoculation with one of three pre-selected Symbiodiniaceae phylotypes, A4, B2 or Breviolum minutum (four jars per phylotype/species). This selection represented a thermally sensitive phylotype (B2), a thermally tolerant phylotype (A4) (see results of thermotolerance experiment) and the phylotype most commonly associated with, or homologous with, Aiptasia NZ1 (B. minutum). The jars were immersed in a water bath at $26^{\circ} \mathrm{C}$, under $\sim 100 \mu$ moles photons $\mathrm{m}^{-2} \mathrm{~s}^{-1}$ provided by $4 \times \mathrm{T} 5$ 54W overhead fluorescent tubes, on a $12 \mathrm{~h}: 12 \mathrm{~h}$ light:dark cycle.

\subsubsection{Inoculation of Aiptasia}

Before inoculation, aposymbiosis in anemones was confirmed by examining 5 randomly selected anemones per jar via fluorescence microscopy. Anemones were inoculated by adding to each jar 3-4 ml of the appropriate Symbiodiniaceae culture at a final cell density of $\sim 3 \times 10^{6} \mathrm{ml}^{-1}$, mixed with a dilute suspension of brine shrimp (Artemia sp. nauplii) to induce phagocytosis. Inoculation was performed weekly, midway through the light cycle, and the water changed and all jars cleaned immediately prior to each inoculation. The anemones were offered no other food during this period.

After four weeks of inoculation, colonisation was confirmed in randomly selected anemones from each jar via fluorescent microscopy (Fig 6.) Different Symbiodiniaceae phylotypes proliferate at different rates in hospite (within the host) due to factors including, but not limited to, sensitivity to nutrient 
limitation within the host and species-specific cell-cycle regulation among Symbiodiniaceae (Tivey et al., 2020). It was therefore necessary to wait another 1-2 weeks after initial detection of symbiont colonistaion to allow for complete and equal colonisation of all anemones before continuing the experiment. Unfortunately, during this period New Zealand went into stage 4 lockdown due to the COVID-19 outbreak and it was no longer possible to access the Victoria University of Wellington biology labs for the following five weeks. Limited maintenance of experimental organisms during this time led to the loss of $\sim 80 \%$ of infected anemones.

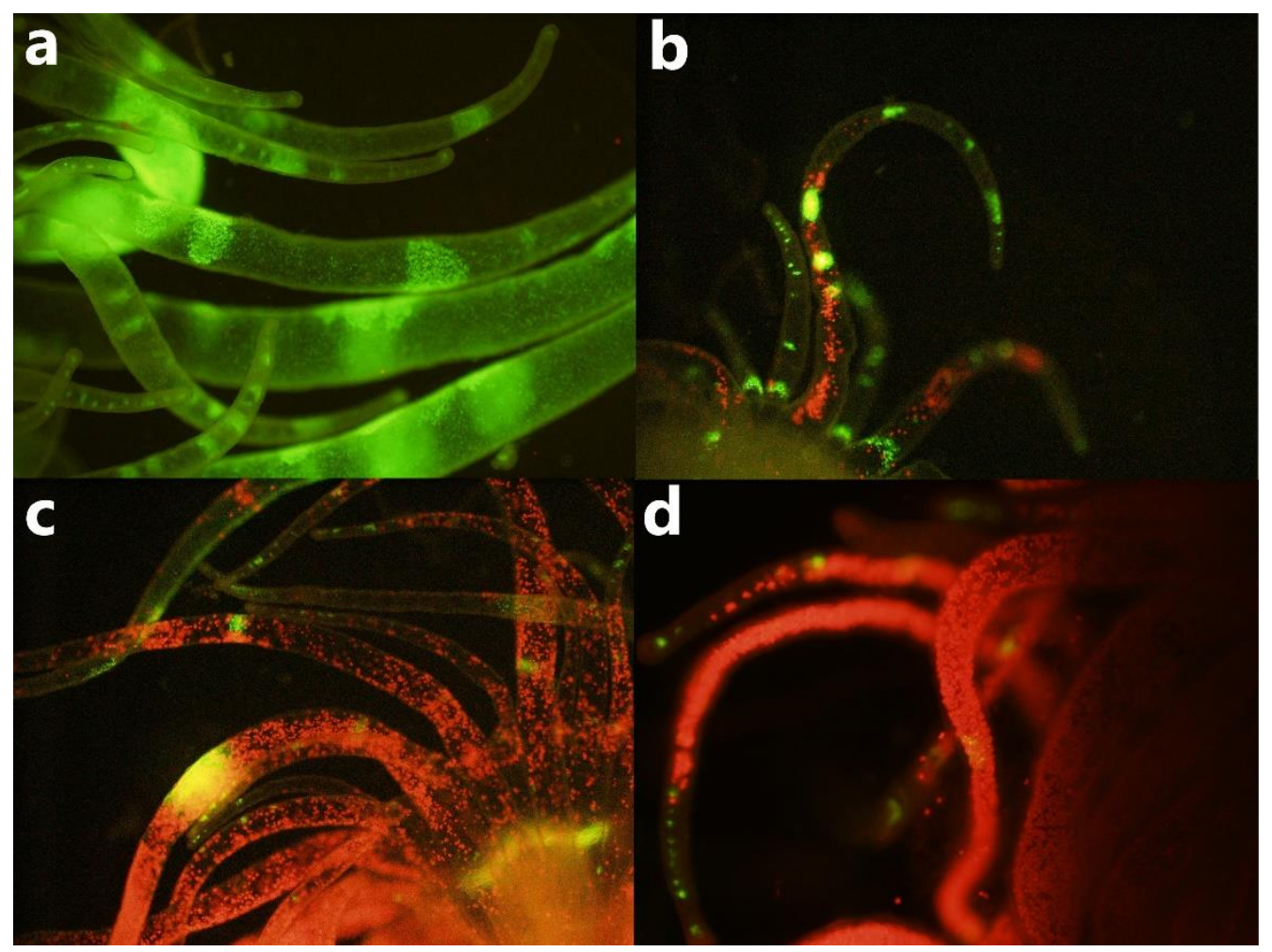

Fig. 6 Fluorescent microscope images showing different degrees of Symbiodiniaceae colonisation in the sea anemone Exaiptasia pallida (Aiptasia). a) Tentacles of an aposymbiotic anemone. The green colour comes from green fluorescent protein (GFP)-like pigments, which are produced by many symbiotic cnidarian species as a photo-protectant (E. G. Smith et al., 2013). b) Partially colonised Aiptasia tentacles. Red/orange points of light are individual Symbiodiniaceae, made visible by chlorophyll autofluorescence. c-d) Increasing densities of Symbiodiniaceae can be seen as the symbionts proliferate in the gastrodermal cells of the host over time. Images taken under blue light (480nm)

Upon regaining lab access, the surviving anemones were redistributed equally among the jars $(\sim 15$ anemones per jar) and aposymbiotic anemones were added to each jar to a total of 70 per jar, and the inoculation protocol repeated. However, due to time constraints exacerbated by the five-week lockdown it was deemed necessary at this point to cut short the second inoculation process after three weeks and 
remove any remaining aposymbiotic or partially colonized anemones from the experiment. The remaining anemones were redistributed equally among the jars giving a total of $\sim 55,48$ and 46 per jar for B.minutum, A4 and B2 respectively.

\subsubsection{Feeding Regime}

Jars were randomly divided into either high or low nutrient treatment groups, with two jars per treatment per phylotype. High nutrient (HN) treatments were then fed 2-3 $\mathrm{ml}$ of a suspension of live brine shrimp pipetted directly above the oral disc, twice weekly for six weeks, while the low nutrient (LN) groups were starved over the same period of time. All groups remained under the same temperature and light conditions as described in section 2.5.1 and the position of jars rotated daily to provide equal light exposure. All jars were cleaned and the water changed weekly during this period. On the first and last days of the feeding regime ten randomly selected anemones from each jar were relaxed in magnesium chloride $(\mathrm{MgCl})$ before having their oral disc diameter measured under a dissecting microscope to monitor the effects of feeding/starvation on the growth of the anemone.

\subsubsection{Temperature Treatment}

On completion of the six-week feeding regime, the jars were further divided into either high or low temperature (HT or LT) groups, giving four treatment groups per phylotype (HN-HT, HN-LT, LN-HT and LN-LT). The HT groups were moved to a separate water bath, but under the same light regime as described in section 2.5.1, and the temperature raised by $1{ }^{\circ} \mathrm{C}$ every $2-4$ days depending on the outcome of PAM readings (See below). Feeding of HN groups continued twice weekly, along with cleaning and water changes once a week. The LT groups were maintained at $26^{\circ} \mathrm{C}( \pm 0.5)$ throughout the experiment and the temperature of both water baths monitored by HOBO pendants. 


\subsubsection{PAM measurements}

Readings of maximum quantum yield $\left(\mathrm{F}_{\mathrm{V}} / \mathrm{F}_{\mathrm{M}}\right)$ were taken daily using an underwater PAM as described previously, with a modified protocol for working with anemones rather than culture. The optical sensor of the instrument was held against the outside of the jar, covering the area where the basal disc of an anemone was attached on the inside. $\mathrm{F}_{0}$ and $\mathrm{F}_{\mathrm{M}}$ were read using the same light parameters as described previously and $\mathrm{Fv} / \mathrm{F}_{\mathrm{M}}$ determined for the symbionts within that anemone. Readings were taken from four randomly selected anemones per jar per day, two from the bottom half of the jar and two from the top half and the mean quantum yield calculated for each treatment group. All organisms were subjected to a 10 min dark-adaptation period before readings began and jars were read in random order.

\subsubsection{Sampling for protein extraction}

Mean quantum yield was compared between HT and LT groups daily via single factor ANOVA (HNHT vs. HN-LT and LN-HT vs. LN-LT for each phylotype) and the mean of the HT groups calculated as a percentage of their corresponding LT groups. When the mean of the HT groups for a particular phylotype dropped to $\leq 70 \%$ of its corresponding LT, and significant difference between the two was confirmed for two consecutive days, a sample of four randomly selected anemones from each group of that phylotype was taken. All four anemones from each jar were placed together in a single $2.5 \mathrm{ml}$ centrifuge tube with $\sim 1.5 \mathrm{ml}$ of MQ-ASW, immediately frozen in liquid nitrogen and then transferred to a $-80^{\circ} \mathrm{C}$ freezer.

After a further $24 \mathrm{~h}$ at the same temperature, a second sample was taken in the same manner as above, after which all jars of that phylotype were immediately moved to a water bath set to $26^{\circ} \mathrm{C}( \pm 0.5)$ and exposed to recovery light conditions of $\sim 20 \mu$ mole photons $\mathrm{m}^{-2} \mathrm{~s}^{-1}$ provided by full spectrum LED 'SolarOasis' overhead grow lights. Sampling was repeated as above $2 \mathrm{~h}, 8 \mathrm{~h}$ and $24 \mathrm{~h}$ after onset of recovery conditions. PAM readings of quantum yield were taken at $0,2,4,6,8,24$ and $48 \mathrm{~h}$ after onset of recovery conditions.

The single sample of $n=4$ anemones at each time point was less than ideal, as it negated comprehensive 
down-stream statistical analysis. However, owing to the reduced starting number of anemones in each treatment group caused by the lockdown period and the inevitable losses incurred as a result of the experimental conditions, working with limited sample sizes was unfortunately unavoidable in the circumstances.

\subsubsection{Holobiont Protein Extraction}

Holobiont (anemone plus symbiont) samples were thawed and excess salt washed from the samples by vortexing briefly $(10-15 \mathrm{sec})$ in $500 \mu \mathrm{l}$ of $\mathrm{MQ} \mathrm{H}_{2} \mathrm{O}$, followed by 1 min centrifugation at $1000 \mathrm{rcf}$. The supernatant was discarded and $500 \mu \mathrm{l}$ of fresh $\mathrm{MQ} \mathrm{H}_{2} \mathrm{O}$ added to the tubes and samples homogenised for $30 \mathrm{sec}$ using a handheld homogeniser (VDI-12, VWR) set to 50\% output. The resulting homogenate was centrifuged for $10 \mathrm{~min}$ at $1000 \mathrm{rcf}$, and the supernatant, which now contained the host fraction of the homogenate, was transferred to a second $2.5 \mathrm{ml}$ centrifuge tube. The pellet was resuspended in 500 $\mu \mathrm{l}$ of MQ $\mathrm{H}_{2} \mathrm{O}$ and centrifuged for a further $10 \mathrm{~min}$ at $500 \mathrm{rcf}$. This supernatant was added to the tube containing the host fraction from the previous step and the symbiont pellet resuspended in $500 \mu \mathrm{l}$ of MQ $\mathrm{H}_{2} \mathrm{O}$ and centrifuged for a further $5 \mathrm{~min}$ at $500 \mathrm{rcf}$. The final supernatant was discarded and the pellet resuspended in $500 \mu \mathrm{l}$ of ice-cold lysis buffer $(100 \mathrm{mM}$ Tris, $100 \mathrm{mM} \mathrm{NaCl}, 10 \mathrm{mM}$ EDTA + a final concentration of 5\% SDS). Samples were immediately transferred to an $85^{\circ} \mathrm{C}$ heating block for 10 min to denature endogenous proteases, then sonicated as described previously before addition of a final concentration of 2-ME. The samples were again heated to $85^{\circ} \mathrm{C}$ for $15 \mathrm{~min}$ and centrifuged a final time at 20,000 $\mathrm{rcf}$ for $10 \mathrm{~min}$. The supernatant (algal protein lysate) was transferred to a new $2.5 \mathrm{ml}$ tube and placed in a $-80^{\circ} \mathrm{C}$ freezer, and the pellet (cell debris) discarded. Samples were kept on ice throughout the above process except for when heating and centrifuging, the latter being performed at $4^{\circ} \mathrm{C}$.

Total protein quantification was performed using a Qubit fluorometer as per the manufacturers instructions

Protein concentrations of 20 (from a total of 60) sample lysates were below the required concentration for use in SDS-PAGE analysis $(<0.5 \mu \mathrm{g} / \mu \mathrm{l})$ and it was necessary for these lysates to be purified further. 
To achieve this, $400 \mu \mathrm{l}$ of protein lysate were added to $1.6 \mathrm{ml}$ of $10 \% \mathrm{TCA} /$ Acetone $(10 \% \mathrm{w} / \mathrm{v}$ trichloroacetic acid dissolved in acetone) in a $2.5 \mathrm{ml}$ centrifuge tube. The TCA causes precipitation of proteins in the lysate, allowing for an increase in total protein concentration by ultimately adding a lower volume of lysis buffer. A final concentration of $20 \mathrm{mM}$ 2-ME was added (to aid in the unfolding and precipitation of proteins) and the solution vortexed for $\sim 30 \mathrm{~s}$, before being incubated for $1 \mathrm{~h}$ at $20^{\circ} \mathrm{C}$. The solution was then centrifuged at $10,000 \mathrm{rcf}$ for $5 \mathrm{~min}$ at $4{ }^{\circ} \mathrm{C}$ and the supernatant discarded. The pellet was washed with $1000 \mu \mathrm{l}$ of ice-cold acetone, vortexed for $\sim 10 \mathrm{~s}$ and centrifuged again at $10,000 \mathrm{rcf}$ for $5 \mathrm{~min}$. The above wash process was repeated three times, after which the pellet was allowed to air dry in a sterile fume hood for approximately $20 \mathrm{~min}$. The dried protein pellet was resuspended in $100 \mu \mathrm{l}$ of lysis buffer (concentrations as above) and heated to $85^{\circ} \mathrm{C}$ in a heating block for 1 hour. During this time the sample was removed from the heating block approximately every 10 $\min$ and vortexed for $\sim 15 \mathrm{~s}$, to aid in dissolution of the protein residue, and then returned to the heating block. The sample was centrifuged a final time at 20,000 $\mathrm{rcf}$ for $5 \mathrm{~min}$ and the supernatant transferred to a new tube and stored in a $-80^{\circ} \mathrm{C}$ freezer.

A pooled lysate was made from $5 \mu$ of each individual lysate and its protein concentration determined as the mean of five replicate samples checked in triplicate with a Qubit fluorometer

\subsubsection{Western Blotting and Optical Density Analysis}

SDS-PAGE was performed as described previously (see section 2.3). Briefly, proteins were separated according to weight via SDS-PAGE and transferred to a PVDF membrane. However, the relatively low yield of protein from many of the lysates precluded the running of multiple blots to determine appropriate D1-STD curves for each sample. Therefore, samples were ranked from highest to lowest total protein concentration as determined by Qubit fluorometry and divided into seven groups of eight and one group of four, with each group representing samples of similar total protein content. Sample groups were then loaded together on the same gel, with individual samples loaded in random order. This method of sample grouping was deemed appropriate under the circumstances, as groups of anemones which gave similar Symbiodiniaceae total protein yields were likely to be hosting similarly 
robust symbiont populations. Working under the assumption that loss of functional PSII reaction centres, and thus D1protein, due to stressful conditions leads to photoinhibition and symbiont expulsion from the cnidarian host (R Hill et al., 2004; D. J. Smith et al., 2005; McGinley et al., 2012; Jeans et al., 2014), symbionts extracted from anemones with low symbiont yields would likely have a correspondingly low concentration of D1 protein. The membrane was blocked, then incubated with a primary antibody $\left(1^{\circ} \mathrm{AB}\right.$, Agrisera, AS05 084) raised against D1 protein, then incubated with a horse radish peroxidase (HRP) conjugated secondary antibody $\left(2^{\circ} \mathrm{AB}\right.$, Agrisera, AS10 668) raised against the $1^{\circ} \mathrm{AB}$. The membrane was imaged with a charge coupled device (CCD) imager (Amersham Imager 600, Amersham) using enhanced chemiluminescence to determine optical density (OD) of the target protein, then stained with Fast Green FC and imaged with a laser scanner (FLA-5100 Fluor Imager, Fujifilm, settings as described previously) to determine mean optical density (MOD) of total protein concentration.

Normalisation of total and target protein was carried out as described previously, except protein concentration was representative of single samples of four anemones each, as opposed to the mean of four biological replicates. In cases where sample target protein OD fell below the range of the D1-STD curve, a second order polynomial regression model was extrapolated downwards from the apparent linear regression. The extrapolated OD values were given as the lowest recorded OD of the D1-STD, divided by the average of the divisors from the two ODs above it. The corresponding concentrations for each extrapolated OD was half that of the next highest concentration. The results obtained from this method of extrapolation were not considered to be a true measurement of the target protein concentration in samples that fell outside of the D1-STD curve, but were instead used to improve resolution when visualising differences in target protein abundance between low concentration samples.

\subsection{Data Analysis}

All data were analysed using R-studio statistical software and checked for assumption of normality using univariate Shapiro-Wilk normality tests. Tests were run against data grouped by high and low nutrient and high and low temperature treatment for each phylotype, with individual time points 
classed as variables. Where assumptions of normality were not met, data were analysed using

Kruskal-Wallis non-parametric one-way ANOVA and Dunn's pair-wise comparison using Bonferroni correction. 


\section{Results}

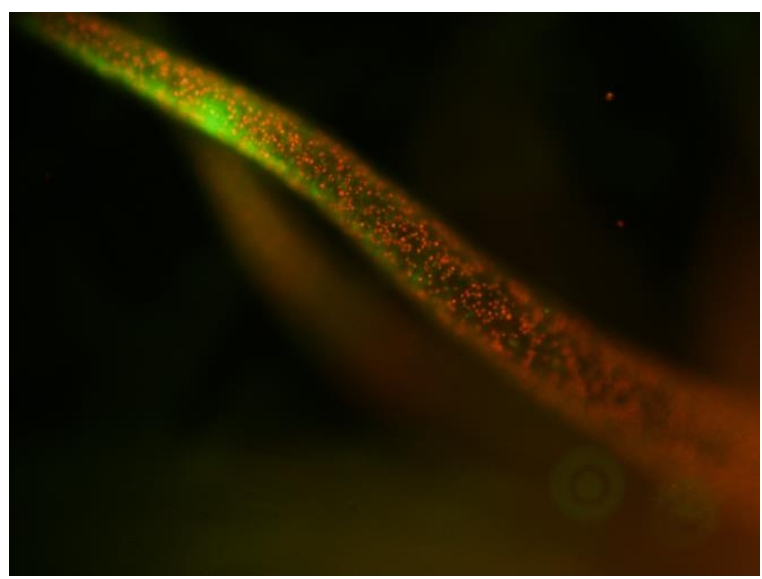

Aiptasia tentacle hosting phylotype B2

\subsection{Thermotolerance Experiment}

Thermotolerance in cultured Symbiodiniaceae species Breviolum minutum and Durusdinium trenchii, and phylotypes A4, B2 and $\mathrm{C} 1$ was determined as the temperature at which a sustained significant difference in maximum quantum yield $\left(\mathrm{F}_{\mathrm{V}} / \mathrm{F}_{\mathrm{M}}\right)$ was observed between cultures maintained at $26^{\circ} \mathrm{C}$ (control) and cultures subjected to a gradual increase in temperature $\left(26-33^{\circ} \mathrm{C}\right.$, temperature treatment). Phylotype B2 was the first to show significant difference between treatment groups (Fig. 3.1b), with mean $\mathrm{F}_{\mathrm{V}} / \mathrm{F}_{\mathrm{M}}$ of the temperature treatment dropping to $93.5 \%{ }^{1 *}$ of the control after $48 \mathrm{~h}$ at $28^{\circ} \mathrm{C}$, reaching a low of 0.522 or $88 \% \%^{2}$ of the control after $96 \mathrm{~h}$ at $28^{\circ} \mathrm{C}$. This difference was lost after the temperature treatment had "recovered" at $26^{\circ} \mathrm{C}$ for $48 \mathrm{~h}$.

The temperature treatment of $D$. trenchii was the next to show departure from the control (Fig. 3.1e), dropping to $66 \%{ }^{3}$ of the latter after $48 \mathrm{~h}$ at $31{ }^{\circ} \mathrm{C}$ and down to $65 \%{ }^{4}$ after $96 \mathrm{~h}$ at the same temperature. Recovery was slower for $D$. trenchii compared to B2, taking $72 \mathrm{~h}$ at $26^{\circ} \mathrm{C}$ to lose the difference between treatment groups. However, this loss of difference between $D$. trenchii treatments after $72 \mathrm{~h}$ was as likely due to a drop in $\mathrm{F}_{\mathrm{V}} / \mathrm{F}_{\mathrm{M}}$ of the control treatment as to an increase in $\mathrm{F}_{\mathrm{V}} / \mathrm{F}_{\mathrm{M}}$ of the temperature treatment.

* Superscripted numbers refer to statistically significant outcomes. Test parameters presented in results appendix 3.6 
Phylotypes A4 and $\mathrm{C} 1$ both showed significant departure at $33^{\circ} \mathrm{C}$ (Fig. 3.1a \& c, respectively), with the temperature treatment of $\mathrm{C} 1$ falling to $63 \%^{5}$ of the control after $24 \mathrm{~h}$ and to $56 \%^{6}$ after $72 \mathrm{~h}$, while the A4 temperature treatment took $48 \mathrm{~h}$ at $33^{\circ} \mathrm{C}$ to reach $86 \%^{7}$ of the control, down to $81 \%^{8}$ after $96 \mathrm{~h}$. While phylotype $\mathrm{A} 4$ recovered after $48 \mathrm{~h}$ at $26^{\circ} \mathrm{C}$, no recovery was observed in $\mathrm{C} 1$ after 5 days at the same temperature.

B. minutum showed no sustained differences (longer than $48 \mathrm{~h}$ ) between treatment groups (Fig. 3.1d), even after $72 \mathrm{~h}$ at $33^{\circ} \mathrm{C}$. However, for much of the experiment the temperature treatment had a higher $\mathrm{F}_{\mathrm{V}} / \mathrm{F}_{\mathrm{M}}$ than the control, and both treatments showed somewhat cyclical fluctuations in $\mathrm{F}_{\mathrm{V}} / \mathrm{F}_{\mathrm{M}}$ over time. It was therefore decided to end the experiment for B. minutum, and A4 and B2 were selected as the high and low thermotolerance Symbiodiniaceae phylotypes respectively on which further experimentation would be performed. 


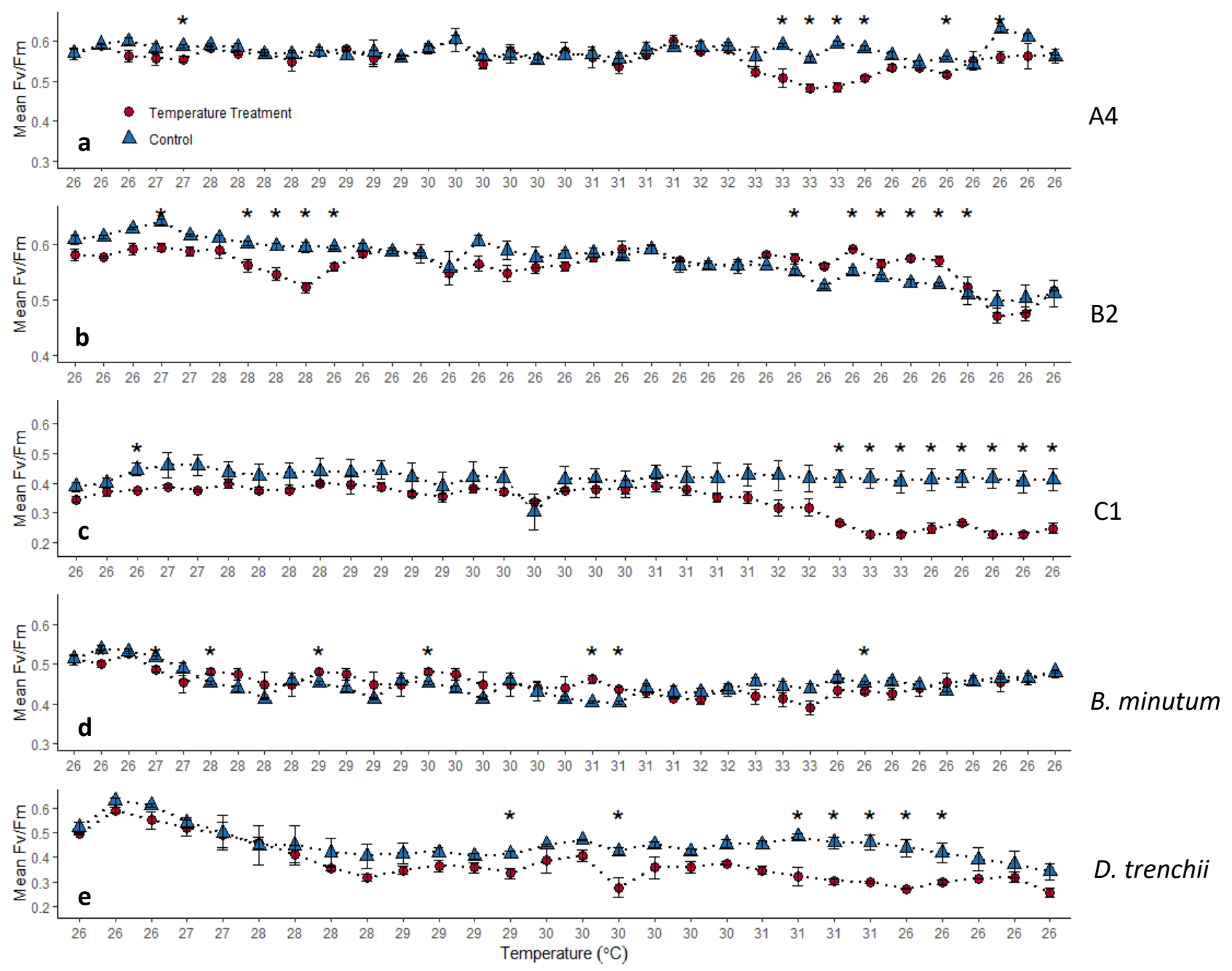

Fig. 3.1 Changes in maximum quantum yield $\left(F_{V} / F_{M}\right)$ as a result of temperature increase in Symbiodiniaceae phylotypes $A 4$ (a), B2 (b) and C1 (c), and species B. minutum (d) and D. trenchii (e). Temperature treatments (red circles) were exposed to increasing temperatures (starting at $26^{\circ} \mathrm{C}$ ), while control treatments (blue triangles) were maintained at $26^{\circ} \mathrm{C}$. Each tick on the $x$-axis represents $24 \mathrm{~h}$. Asterisks above plots indicate significant difference in $\mathrm{Fv}_{\mathrm{v}} / \mathrm{F}_{\mathrm{M}}$ between treatment groups (One-way ANOVA, $p<0.05)$. Means shown $\pm \mathrm{SE}$.

\subsection{Culture Experiment}

The influence of nutrient enrichment on values of maximum quantum yield, non-photochemical energydissipation and D1 protein concentration were observed in cultured Symbiodiniaceae subjected to either constant or gradually increasing temperature. 


\subsubsection{Influence of Temperature and Nutrients on Maximum Quantum Yield $\left(\mathbf{F}_{\mathrm{v}} / \mathbf{F}_{\mathrm{M}}\right)$}

Both temperature and nutrient regime were found to influence the maximum quantum yield $\left(\mathrm{F}_{\mathrm{V}} / \mathrm{F}_{\mathrm{M}}\right)$ of Symbiodiniaceae phylotypes A4 (thermotolerant) and B2 (thermally sensitive). In all treatments where temperature was gradually increased from $26-31^{\circ} \mathrm{C}$ (high temperature treatments, $\mathrm{HT}$ ), $\mathrm{F}_{\mathrm{V}} / \mathrm{F}_{\mathrm{M}}$ fell significantly below treatments that were maintained at $26^{\circ} \mathrm{C}$ (low temperature treatments, LT) ${ }^{1}$. The influence of nutrient regime was more pronounced in phylotype A4 than in B2, with high nutrient treatments of $\mathrm{A} 4$ showing increased $\mathrm{F}_{\mathrm{V}} / \mathrm{F}_{\mathrm{M}}$ relative to all other treatments of either phylotype ${ }^{2}$.

\subsubsection{Changes in $F_{V} / F_{M}$ of Symbiodiniaceae Phylotype $A 4$}

The low nutrient treatments of phylotype $\mathrm{A} 4$ showed stable $\mathrm{F}_{\mathrm{V}} / \mathrm{F}_{\mathrm{M}}$ in the early stages of the experiment, with no significant difference observed between the low temperature (LT) treatment and the high temperature (HT) treatment until $\mathrm{HT}$ had been exposed to $31^{\circ} \mathrm{C}$ for $24 \mathrm{~h}$ (Fig. 3.2a). At this time, $\mathrm{F}_{\mathrm{V}} / \mathrm{F}_{\mathrm{M}}$ of HT fell to $72 \%{ }^{1}$ of LT, and continued to drop over the following $48 \mathrm{~h}$ to reach a low of $0.28( \pm 0.015)$, or $60 \%{ }^{2}$ of LT. In contrast, HT and LT of the high nutrient treatments remained equal until HT had been exposed to $31^{\circ} \mathrm{C}$ for $48 \mathrm{~h}$ (Fig. 3.2b), with HT reaching a low of $0.464( \pm 0.009)$, or $86 \%{ }^{3}$ of LT after a further $24 \mathrm{~h}$ at $31^{\circ} \mathrm{C}$. Immediately following this, all treatments entered recovery conditions $\left(26^{\circ} \mathrm{C}\right.$ and $20 \mu \mathrm{mol}$ photons $\left.\mathrm{m}^{-2} \mathrm{~s}^{-1}\right)$, and the chloroplast antibiotic lincomycin was added to one half of the flasks from each treatment $(+\mathrm{L})$.

Following two hours of recovery, $\mathrm{F}_{\mathrm{V}} / \mathrm{F}_{\mathrm{M}}$ of $\mathrm{HT}$ and $\mathrm{HT}+\mathrm{L}$ from the low nutrient treatment had increased by $40 \%{ }^{4}$ and $32 \%{ }^{5}$ respectively (Fig. 3.3a), with an overall increase of $58 \%^{6}$ and $36 \%^{7}$ respectively after $24 \mathrm{~h}$ recovery. By comparison, both $\mathrm{HT}$ and $\mathrm{HT}+\mathrm{L}$ from the high nutrient treatment recovered $16 \%^{8}$ in the first two hours (Fig. 3.3b), rising to an overall recovery of $17 \%^{9}$ and $18 \%^{10}$ respectively after $24 \mathrm{~h}$.

When comparing between nutrient treatments up to the onset of recovery conditions, $\mathrm{F}_{\mathrm{V}} / \mathrm{F}_{\mathrm{M}}$ of $\mathrm{HT}$ and LT from the high nutrient treatment (HN-HT and HN-LT) were at all times higher than $\mathrm{HT}^{11}$ and $\mathrm{LT}^{12}$ from the low nutrient treatment respectively (LN-HT and LN-LT). After two hours recovery, no difference was observed between $\mathrm{HN}-\mathrm{LT}+\mathrm{L}$ and $\mathrm{LN}-\mathrm{LT}+\mathrm{L}$, but following $24 \mathrm{~h}$ recovery, all $\mathrm{HN}$ temperature treatments were again significantly higher than their respective LN temperature treatments 
(Fig. 3.4a-c). No significant difference was observed between lincomycin and non-lincomycin treatments after $24 \mathrm{~h}$.

\subsubsection{Changes in $\mathbf{F}_{\mathrm{V}} / \mathbf{F}_{M}$ of Symbiodiniaceae Phylotype $\mathbf{B} 2$}

Prior to recovery conditions, $\mathrm{F}_{\mathrm{V}} / \mathrm{F}_{\mathrm{M}}$ of phylotype $\mathrm{B} 2$ showed a similar trend to that of phylotype $\mathrm{A} 4$, with little difference observed between HT and LT in either of the nutrient treatments. After $24 \mathrm{~h}$ at $31^{\circ} \mathrm{C}$, LN-HT dropped to $91 \%{ }^{1}$ of LN-LT, and after $72 \mathrm{~h}$ at the same temperature LN-HT reached a low of $0.326( \pm 0.01)$, or $68 \%^{2}$ of LN-LT (Fig. 3.2c). Over this same period HN-HT fell to 0.392 , or $81 \%^{3}$ of HN-LT (Fig. 3.2d).

Notably, after $48 \mathrm{~h}$ at $31^{\circ} \mathrm{C}$, LN-HT of phylotype B2 was $15 \%{ }^{4}$ higher than LN-HT of phylotype A4. In contrast, $\mathrm{HN}-\mathrm{HT}$ of $\mathrm{B} 2$ was on average $15 \%{ }^{5}$ lower than $\mathrm{HN}-\mathrm{HT}$ of $\mathrm{A} 4$ throughout the experiment. Following two hours of recovery, both LN-HT and LN-HT+L of B2 had increased by $\sim 33 \%{ }^{6,7}$, with a total increase of $42 \%{ }^{8}$ and $46 \%{ }^{9}$ respectively over $24 \mathrm{~h}$ (Fig. 3.3c). For the high nutrient treatments, an increase of $26 \%{ }^{10}$ and $27 \%{ }^{11}$ was observed in HN-HT and HN-HT+L respectively following the first two hours of recovery only, after which no further change was observed for the remainder of the recovery period (Fig. 3.3d).

From $72 \mathrm{~h}$ after the start of the experiment until $24 \mathrm{~h}$ before the onset of recovery conditions, all low nutrient treatments were below their corresponding high nutrient counterparts ${ }^{12}$. Immediately before entering recovery, significant difference was observed only between $\mathrm{LN}-\mathrm{HT}$ and $\mathrm{HN}-\mathrm{HT}^{13}$, and from two hours of recovery until the end of the $24 \mathrm{~h}$ recovery period, no differences were observed between any of the low nutrient treatments and their high nutrient counterparts, or between lincomycin and nonlincomycin treatments.

When comparing between phylotypes, high nutrient treatments of A4 averaged $\sim 9 \%{ }^{14}$ above $\mathrm{HN}$ of B2 throughout recovery, while the opposite was true with low nutrient treatments, with B2 LN on average $\sim 9 \%{ }^{15}$ higher than A4 LN. 


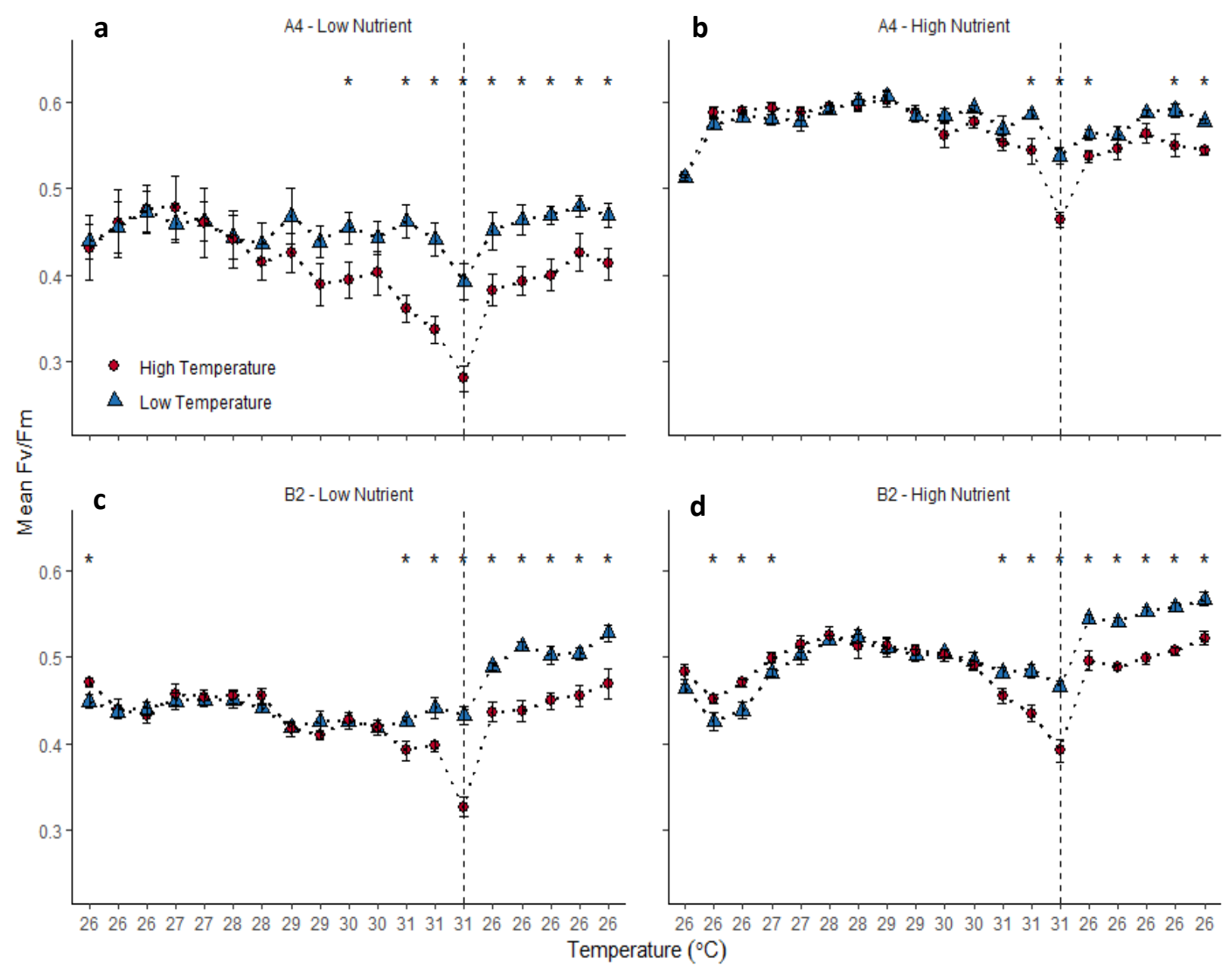

Fig. 3.2 Changes in $F_{V} / F_{M}$ in response to temperature. Top Row: Symbiodiniaceae phylotype $A 4$, low (a) and high (b) nutrient treatments. Bottom row: Symbiodiniaceae phylotype B2, low (c) and high (d) nutrient treatments. Red circles = high temperature treatment $\left(26-31^{\circ} \mathrm{C}\right)$, blue triangles $=$ low temperature treatments $\left(26^{\circ} \mathrm{C}\right)$. Vertical dashed lines indicate start of recovery period. Values on the $\mathrm{x}$-axis refer to high temperature treatments only and represent $24 \mathrm{~h}$ intervals prior to onset of recovery and 2, 4, 6, 8 and 24 h post recovery. Asterisks above plots denote significant difference between mean $F_{v} / F_{M}$ of low and high temperature treatments within the same phylotype and nutrient group $(p<0.05, n=8)$. Means shown $\pm S E$, $\mathrm{n}=8$. 


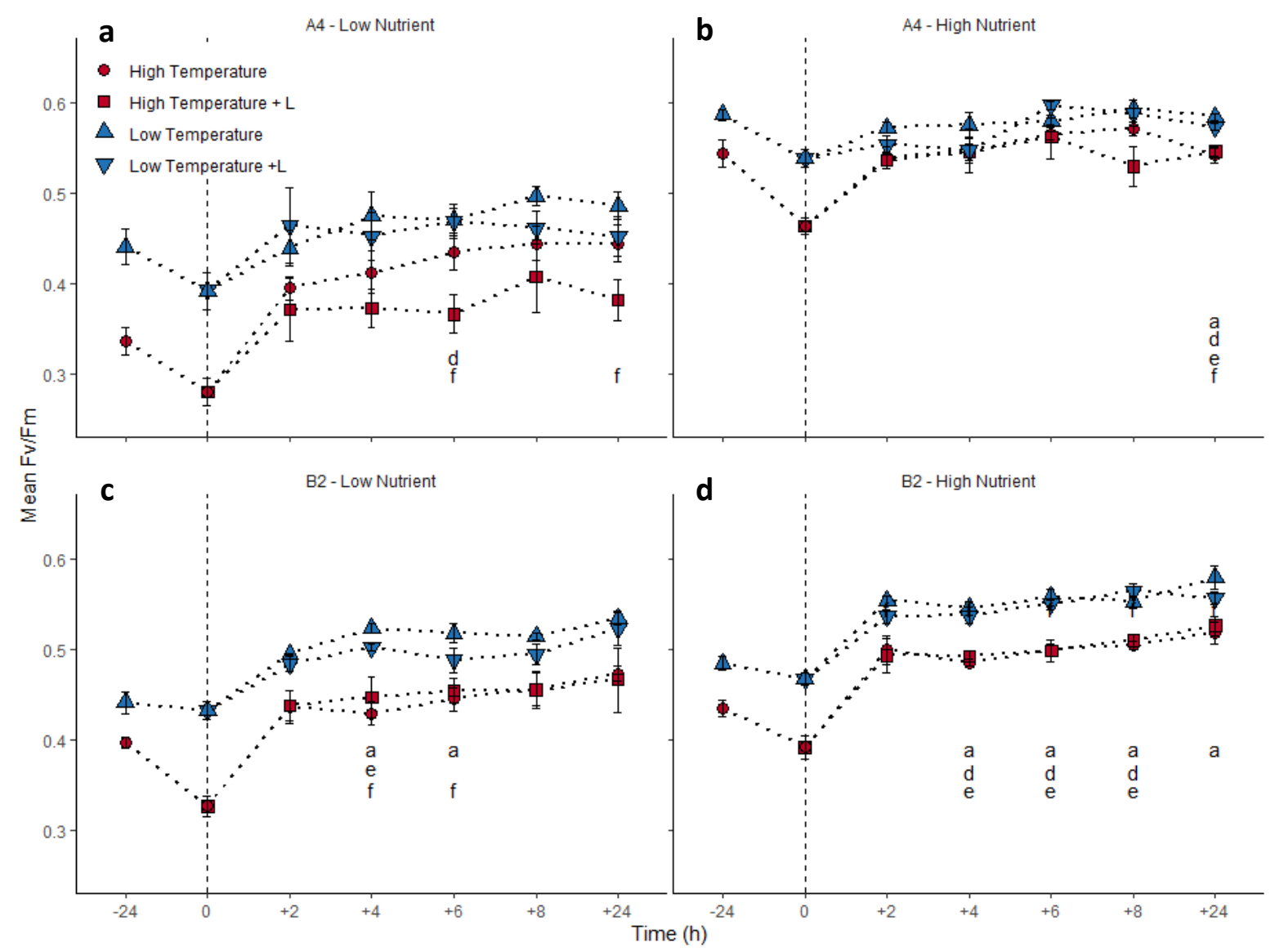

Fig. 3.3 Changes in $\mathrm{F}_{v} / \mathrm{F}_{\mathrm{M}}$ during recovery with all treatments at $26^{\circ} \mathrm{C}$. Top row: Symbiodiniaceae phylotype $\mathrm{A} 4$ low (a) and high (b) nutrient treatments. Bottom row: Symbiodiniaceae phylotype B2 low (c) and high (d) nutrient treatments. Red circles $=$ high temperature treatment $\left(31^{\circ} \mathrm{C}\right)$, red squares $=$ high temperature treatment + lincomycin, blue triangles = low temperature treatment $\left(26^{\circ} \mathrm{C}\right)$, blue inverted triangles = low temperature treatment + lincomycin. Vertical dashed line indicates start of recovery conditions $\left(26^{\circ} \mathrm{C}\right.$ and $\sim 20 \mu \mathrm{mol}$ photons $\left.\mathrm{m}^{-2} \mathrm{~s}^{-1}\right)$ and addition of lincomycin to $+\mathrm{L}$ treatments. Values of $y$-axis are time in hours from start of recovery conditions. Letters below the plots indicate significant difference found between treatments via post hoc Tukey's HSD tests $(\alpha=0.05, n=4)$ where $a=L T$ vs. $H T, b=L T+L$ vs. $L T, c=H T+L$ vs. $H T, d=$ $L T+L$ vs. $H T+L, e=L T+L$ vs. $H T, f=L T$ vs. $H T+L$. Means shown $\pm S E, n=4$. 
A4

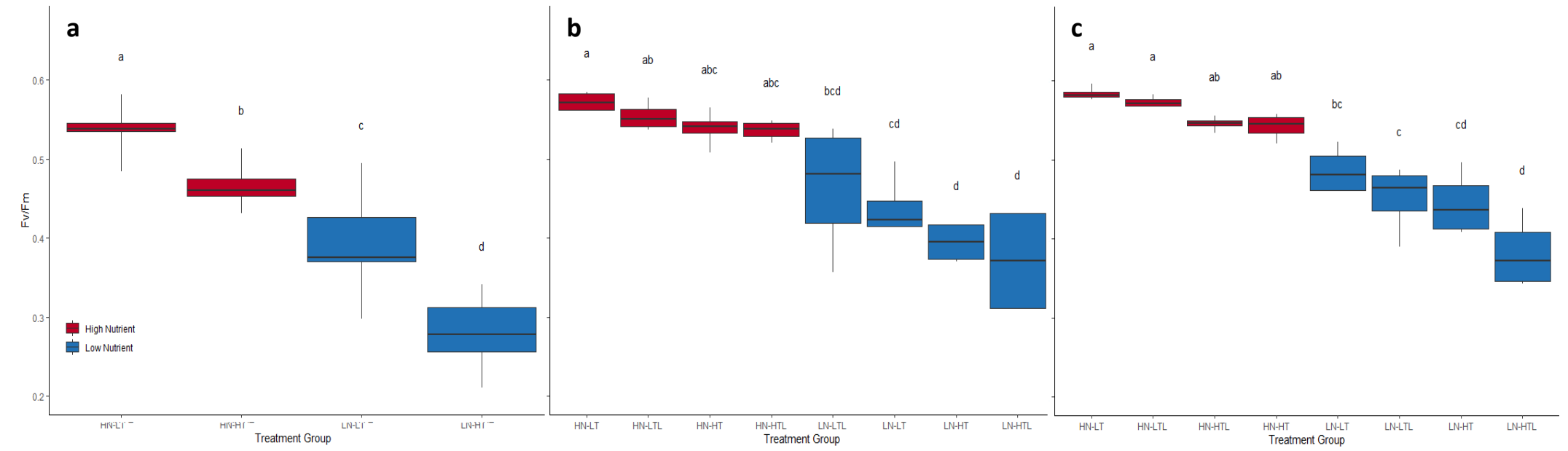

B2

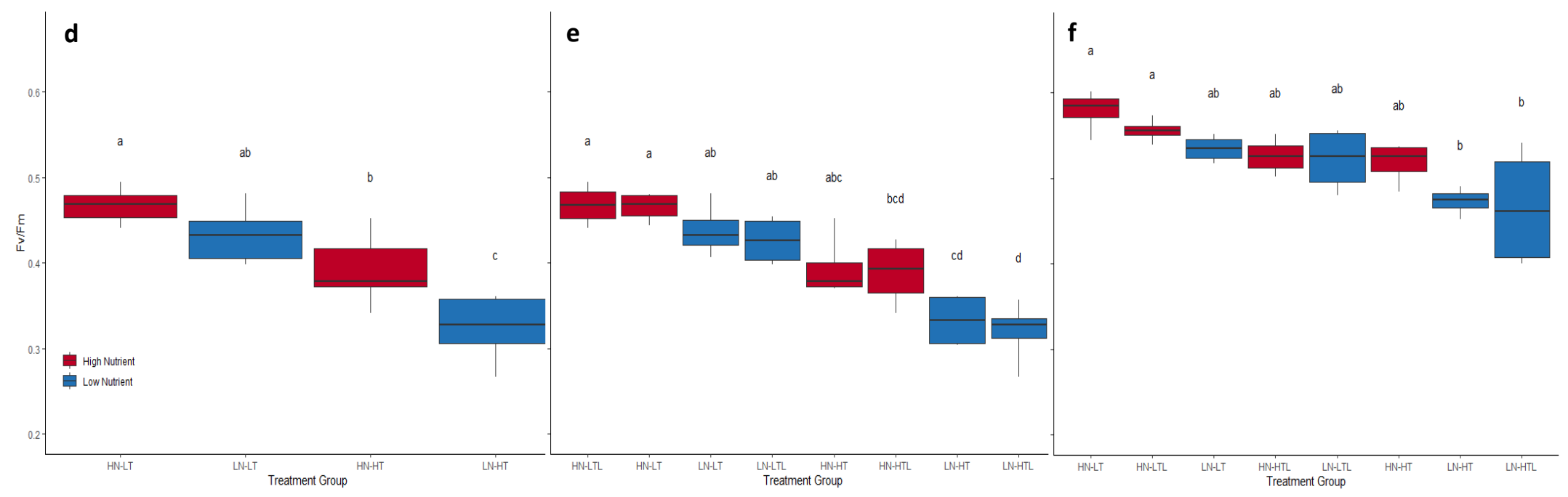

Fig. 3.4 Difference in mean $\mathrm{F}_{\mathrm{V}} / \mathrm{F}_{\mathrm{M}}$ between temperature treatments of opposing nutrient groups during recovery. Times shown are immediately before recovery, after two hours of recovery and after $24 \mathrm{~h}$ recovery (a-c phylotype $\mathrm{A} 4$ and $\mathbf{d}$-f phylotype B2 respectively). Boxes show mean $\mathrm{F}_{\mathrm{V}} / \mathrm{F}_{\mathrm{M}}$, upper and lower quartile and $\pm S E$ for each treatment group. Letters above boxes indicate statistically distinct groups within each phylotype and at each time point (Tukey's HSD post-hoc test, $\alpha=0.05, \mathbf{a}, \mathbf{d} n=8, \mathbf{b}, \mathbf{c}, \mathbf{e}, \mathbf{f} n=4$ ) 


\subsection{Influence of Temperature and Nutrient Regime on Fluorescence Yield as a Response to Short-term Light Exposure.}

Dark-light induction curves were analysed to determine changes in effective PSII quantum yield, nonphotochemical quenching and non-regulated heat dissipation (Y(II), Y(NPQ) and Y(NO) respectively) as a response to actinic light exposure under low and high nutrient and low $\left(26^{\circ} \mathrm{C}\right)$ and high $\left(31^{\circ} \mathrm{C}\right)$ temperature conditions. Distinct differences were observed between phylotypes A4 and B2 with respect to the curve shape of individual parameters, as well as varying degrees of difference in curve shape between nutrient and temperature treatments within each phylotype.

\subsubsection{Fluorescence Curves of Phylotype A4}

Phylotype A4 showed considerable variation in parameter curve shape between nutrient and temperature treatments over the course of the induction procedure (Fig. 3.5). Between low temperature treatments, the Y(NPQ) value of A4 HN-LT was $76 \%^{1}( \pm 4.6)$ that of A4 LN-LT for the first four minutes of the actinic period, dropping to $37 \%^{2}( \pm 10.2)$ for the final four minutes (Fig. 3.5, a \& b). The Y(II) value of LN-LT was $57 \%^{3}( \pm 1.8)$ that of HN-LT throughout the actinic period. Between low nutrient treatments, Y(NPQ) of LN-HT was below that of LN-LT for the first two minutes of the actinic period only $\left(78 \%^{4}( \pm 1.8)\right.$ and $88 \%^{5}( \pm 1.5)$ respectively), before becoming equal for the remainder, while Y(II) of LN-HT was $69 \%^{6}( \pm 2.1)^{6}$ of LN-LT throughout the actinic period (Fig. 3.5, b \& d). Between the high nutrient treatments, Y(NPQ) of HN-LT was $34 \%^{7}( \pm 11.7)$ of HN-HT for the final three minutes of the actinic period, while Y(II) of HN-HT averaged $68 \%^{8}( \pm 0.2)$ of HN-LT over the final two minutes (Fig. 3.5, a \& c). Between the high temperature treatments, there was no significant difference in Y(NPQ), but Y(II) of LN-HT averaged $48 \%{ }^{9}( \pm 0.3)$ that of HN-HT throughout the actinic period. Of note, $\mathrm{Y}(\mathrm{NO})$ of $\mathrm{LN}-\mathrm{HT}$ was $31 \%^{10}( \pm 0.9)$ higher than that of HN-HT throughout the entire D-L induction procedure (Fig 3.5, c \& d). 


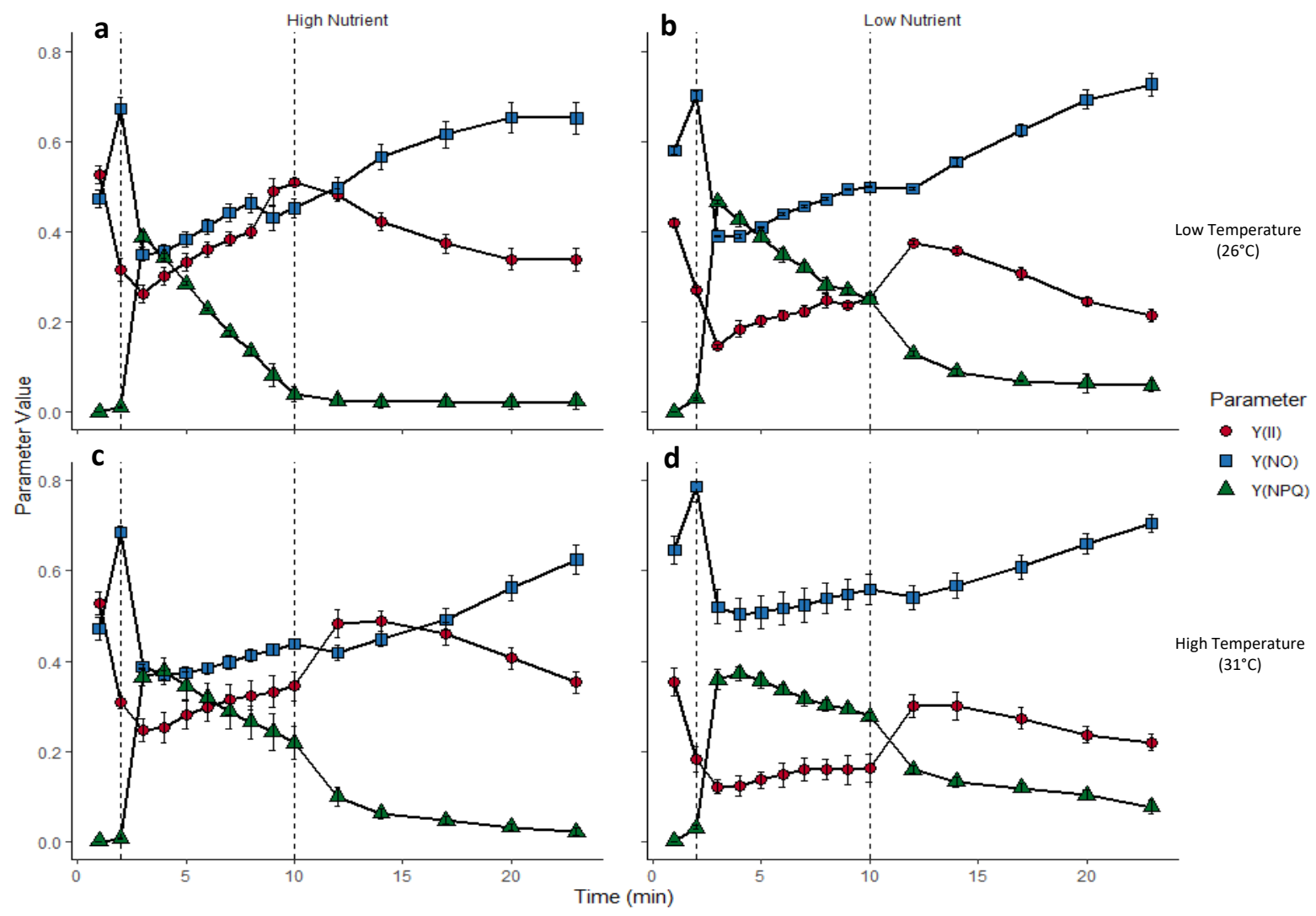

Fig. 3.5 Dark-light induction curves showing changes in PSII effective quantum yield $(Y(I I))$, non-photochemical quenching, $(\mathrm{Y}(\mathrm{NPQ}))$ and non-regulated heat dissipation $(\mathrm{Y}(\mathrm{NO})$ in Symbiodiniaceae phylotype $\mathrm{A} 4$ as a response to actinic light exposure $\left(167 \mu \mathrm{mol}\right.$ photons $\left.\mathrm{m}^{-2} \mathrm{~s}^{-1}\right)$. Treatments are a) High Nutrient - Low Temperature $\left(26^{\circ} \mathrm{C}, \mathrm{n}=4\right)$, b) Low Nutrient - Low Temperature $\left(26^{\circ} \mathrm{C}, \mathrm{n}=3\right)$, c) High Nutrient - High Temperature $\left(31^{\circ} \mathrm{C}, \mathrm{n}=4\right)$, d) Low Nutrient - High Temperature $\left(31^{\circ} \mathrm{C}, \mathrm{n}=5\right)$. Vertical dashed lines indicate beginning and end of actinic light exposure period. Means shown \pm SE.

\subsubsection{Fluorescence Curves of Phylotype B2}

Less variation in parameter value was observed between nutrient and temperature treatments of phylotype B2 (Fig. 3.6). Between low temperature treatments, no difference in Y(NPQ) was observed, while Y(II) of LN-LT was $80 \%^{1}( \pm 1.2)$ that of HN-LT throughout the actinic period (Fig. 3.6 a \& b). Between low nutrient treatments, there was also no difference in Y(NPQ), but Y(II) of LN-HT dropped to $82 \%{ }^{2}( \pm 1.1)$ of LN-LT after the sixth minute of the actinic period (Fig 3.6, b \& d). Between high nutrient treatments, no difference was observed between any of the three parameters throughout the procedure (Fig. 3.6, a \& c), while between high temperature treatments Y(II) of LN-HT was 74\% ${ }^{3}$ $( \pm 1.1)$ that of HN-LT for the third and fourth minute of the actinic period only (Fig. 3.6, c \& d). 


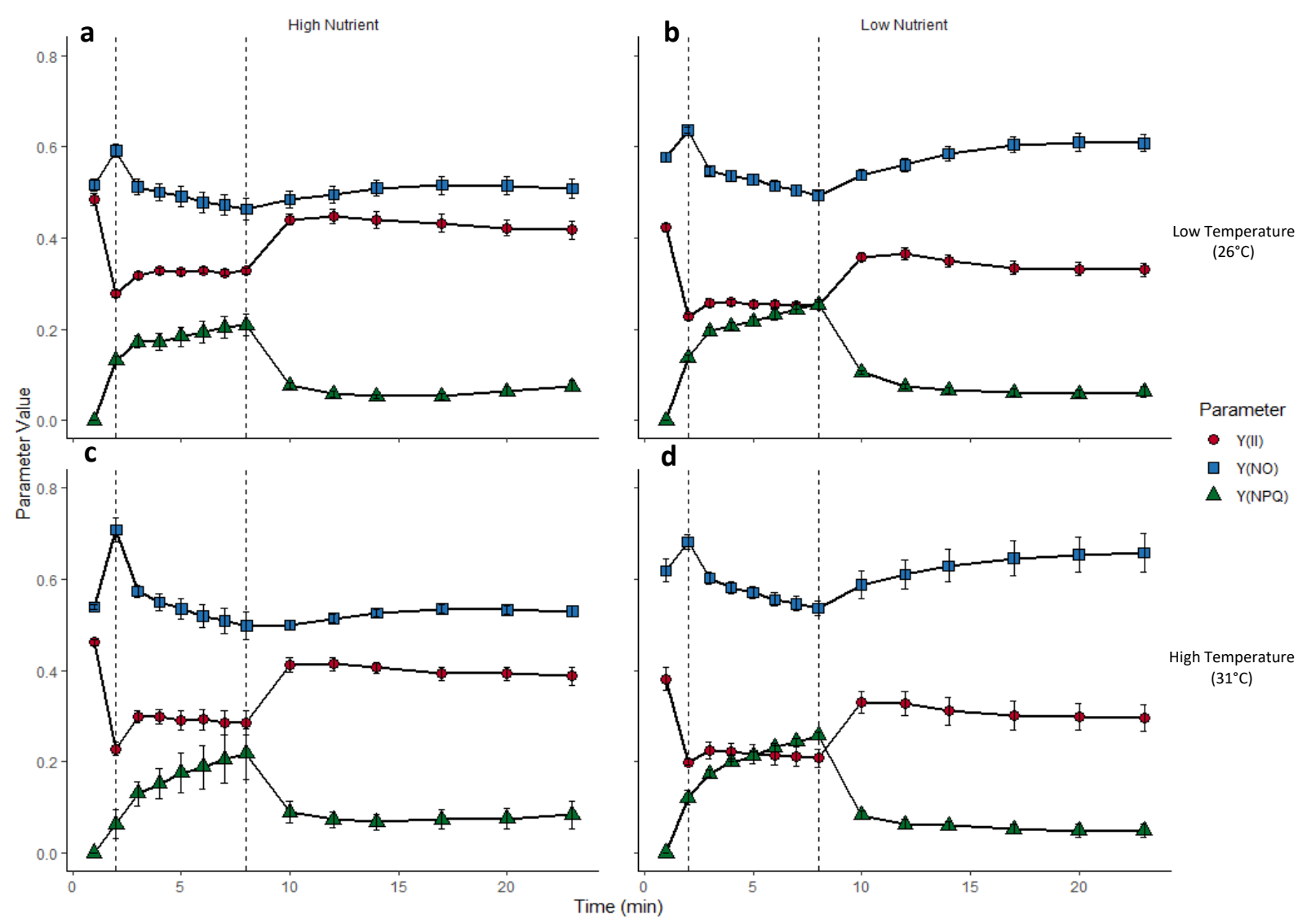

Fig. 3.6 Dark-light induction curves showing changes in PSII effective quantum yield $(Y(I I))$, non-photochemical quenching, $(\mathrm{Y}(\mathrm{NPQ}))$ and non-regulated heat dissipation $(\mathrm{Y}(\mathrm{NO})$ in Symbiodiniaceae phylotype $\mathrm{B} 2$ as a response to actinic light exposure $\left(167 \mu \mathrm{mol}\right.$ photons $\left.\mathrm{m}^{-2} \mathrm{~s}^{-1}\right)$. Treatments are a) High Nutrient - Low Temperature $\left(26^{\circ} \mathrm{C}, \mathrm{n}=5\right)$, b) Low Nutrient - Low Temperature $\left(26^{\circ} \mathrm{C}, \mathrm{n}=7\right)$, c) High Nutrient - High Temperature $\left(31^{\circ} \mathrm{C}, \mathrm{n}=4\right)$, d) Low Nutrient - High Temperature $\left(31^{\circ} \mathrm{C}, \mathrm{n}=5\right)$. Vertical dashed lines indicate beginning and end of actinic light exposure period. Means shown $\pm \mathrm{SE}$.

\subsubsection{Y(NPQ) Dynamics}

The maximum value of Y(NPQ) for phylotype A4 was recorded after the first minute of the actinic period in both low temperature treatments, and after the second minute in both high temperature treatments, before declining steadily in all treatments. This is in contrast to phylotype B2, where in all cases the maximum $\mathrm{Y}(\mathrm{NPQ})$ was recorded in the last minute of the actinic period, after rising steadily from the onset of actinic exposure (Table 3.1). 
Table 3.1. Limits of mean values of $\mathrm{Y}(\mathrm{NPQ})$ during actinic exposure. Data shown are minimum and maximum mean $\mathrm{Y}(\mathrm{NPQ})$ values for each treatment, including standard error of the mean and time of observation in minutes after onset of actinic exposure. Fold change between minimum and maximum values are shown only where significant difference between means were found via one-way ANOVA.

\begin{tabular}{|c|c|c|c|c|c|c|c|c|c|c|c|}
\hline \multirow[t]{2}{*}{ Phylotype } & \multirow[t]{2}{*}{ Treatment } & \multicolumn{3}{|c|}{ Minimum Y(NPQ) } & \multicolumn{3}{|c|}{ Maximum Y(NPQ) } & \multirow{2}{*}{$\begin{array}{l}\text { Fold change } \\
\text { (Min.-Max.) }\end{array}$} & \multirow[t]{2}{*}{ D.F } & \multirow{2}{*}{$\begin{array}{c}\text { F- } \\
\text { Statistic }\end{array}$} & \multirow[t]{2}{*}{ P-value } \\
\hline & & Mean & $\pm \mathrm{SE}$ & $\begin{array}{l}\text { Time } \\
\text { (min) }\end{array}$ & Mean & \pm ISE & $\begin{array}{l}\text { Time } \\
\text { (min) }\end{array}$ & & & & \\
\hline A4 & HN-LT & 0.039 & 0.017 & 8 & 0.389 & 0.006 & 1 & 4.8 & 1,6 & 131 & $<0.001$ \\
\hline A4 & $\mathrm{HN}-\mathrm{HT}$ & 0.217 & 0.028 & 8 & 0.377 & 0.021 & 2 & n.s & - & - & - \\
\hline A4 & LN-LT & 0.249 & 0.005 & 8 & 0.465 & 0.003 & 1 & 1.73 & 1,4 & 516 & $<0.001$ \\
\hline A4 & $\mathrm{LN}-\mathrm{HT}$ & 0.277 & 0.007 & 8 & 0.372 & 0.01 & 2 & 1.27 & 1,6 & 18.5 & 0.005 \\
\hline B2 & HN-LT & 0.131 & 0.006 & 1 & 0.209 & 0.018 & 6 & 1.6 & 1,8 & 8.96 & 0.017 \\
\hline B2 & $\mathrm{HN}-\mathrm{HT}$ & 0.063 & 0.023 & 1 & 0.217 & 0.039 & 6 & n.s & - & - & - \\
\hline B2 & LN-LT & 0.137 & 0.005 & 1 & 0.254 & 0.007 & 6 & 1.9 & 1,12 & 82.6 & $<0.001$ \\
\hline B2 & $\mathrm{LN}-\mathrm{HT}$ & 0.12 & 0.011 & 1 & 0.257 & 0.007 & 6 & 2.14 & 1,8 & 59 & $<0.001$ \\
\hline
\end{tabular}

\subsection{Influence of Temperature and Nutrients on D1 Protein Concentration}

Changes in D1 protein concentration in cultured Symbiodiniaceae phylotypes A4 and B2 were examined to determine the influence of nutrients on rates of D1 protein synthesis and turnover, both under heat stress and during recovery. Considerable differences were observed between phylotypes and treatments, with all high temperature treatments of phylotype A4 showing an increase in D1 protein concentration over the $24 \mathrm{~h}$ recovery period. Treatments $\mathrm{HN}-\mathrm{HT}$ and $\mathrm{HN}-\mathrm{HT}+\mathrm{L}$ increased by $184 \%{ }^{1}$ and $84 \%^{2}$ respectively (Fig. 3.7 a-b), while LN-HT and $\mathrm{LN}-\mathrm{HT}+\mathrm{L}$ increased by $375 \%^{3}$ and $171 \%^{4}$ respectively (Fig. 3.7 e-f). No increase was observed in the low temperature treatments of phylotype A4, however D1 protein concentration in both HN-LT and HN-LT+L decreased significantly after two hours of recovery (by $72 \%{ }^{5}$ and $65 \%^{6}$ respectively) before returning to pre-recovery levels after $24 \mathrm{~h}$ (Fig 3.7 c-d). Concentrations of D1 were significantly lower in HN-HT than HN-LT both 24 h before and at the onset of recovery $\left(45 \%^{7}\right.$ and $35 \%^{8}$ respectively)

In contrast, no increase in D1 concentration was observed at the conclusion of the recovery period for any phylotype B2 treatments. With the exception of HN-HT, all high nutrient treatments showed a net decrease from $24 \mathrm{~h}$ pre-recovery to $24 \mathrm{~h}$ post-recovery $\left(-77 \%{ }^{9},-70 \%^{10}\right.$ and $-80 \%^{11}$ for $\mathrm{HN}-\mathrm{HT}+\mathrm{L}, \mathrm{HN}-$ LT and HN-LT+L respectively) (Fig. 3.8 a-d). No significant change was observed for any of the low nutrient treatments (Fig 3.8 e-h). 
No difference in D1 concentration was observed when comparing between temperature treatments within the same nutrient treatment at each time point. Overall, concentrations of D1 protein in phylotype A4 were considerably higher than in B2, with the high and low nutrient treatments of A4 averaging $326 \%{ }^{12}$ and $330 \%{ }^{13}$ those of $\mathrm{B} 2$ respectively.

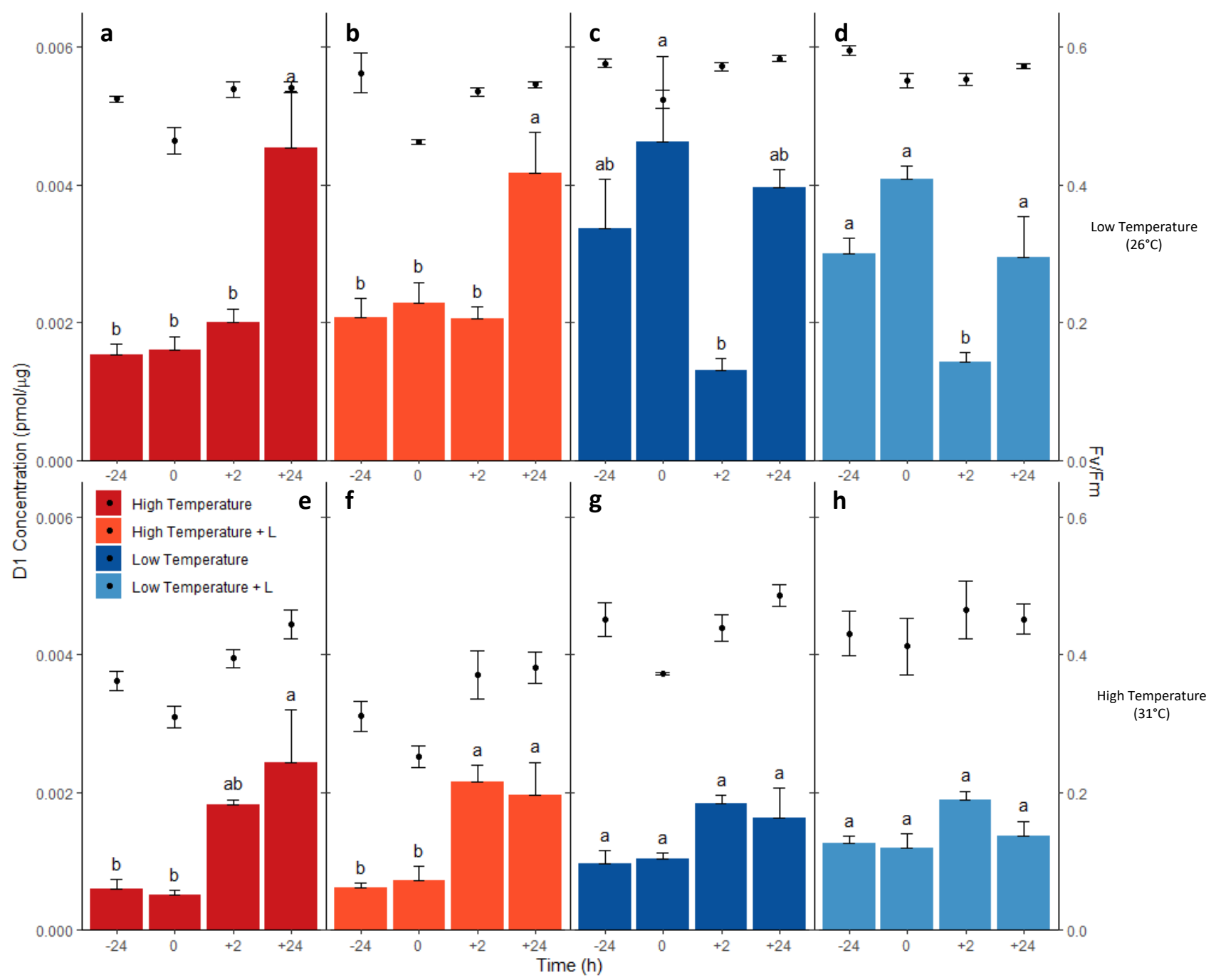

Fig. 3.7 D1 protein content and corresponding $F_{v} / F_{M}$ of cultured Symbiodiniaceae phylotype $A 4$ under differing nutrient and temperature treatments. a-d high nutrient treatments, e-h low nutrient treatments. Bars represent mean D1 protein concentration in pmol $\mathrm{gg}^{-1}$ of total protein $+\mathrm{SE}$ (left y-axis). Points above bars represent corresponding $\mathrm{Fv}_{\mathrm{v}} / \mathrm{F}_{\mathrm{M}}$ at each time point $\pm \mathrm{SE}$ (right $y$-axis). $X$-axis is time in hours from start of recovery conditions. Letters above bars indicate statistically distinct groups of D1 content within individual treatments as determined by Tukey's post hoc HSD tests $(\alpha=0.05, n=4)$ 


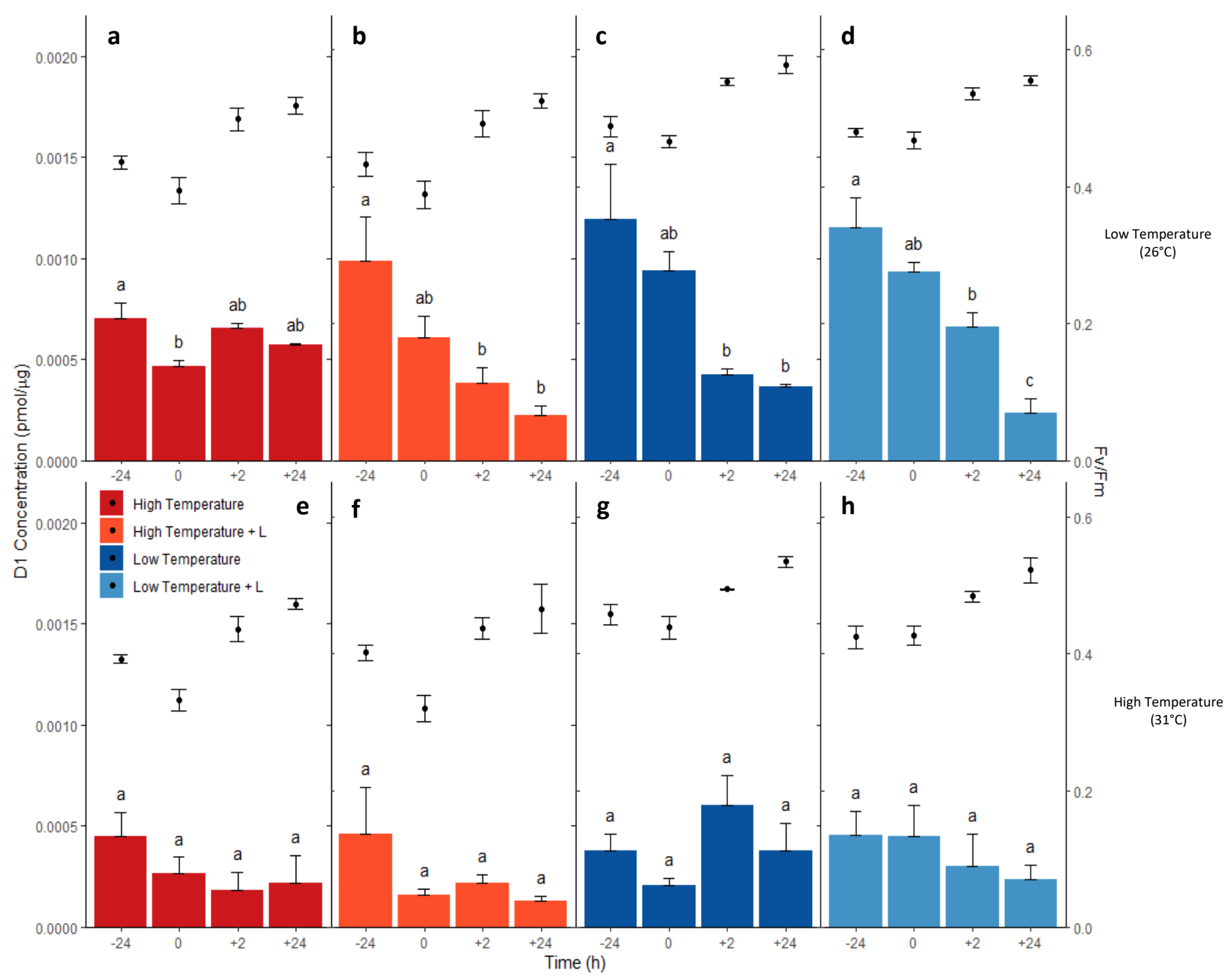

Fig. 3.8 D1 protein content and corresponding $\mathrm{F}_{\mathrm{V}} / \mathrm{F}_{\mathrm{M}}$ of cultured Symbiodiniaceae phylotype B2 under differing nutrient and temperature treatments. a-d high nutrient treatments, e-h low nutrient treatments. Bars represent mean D1 protein concentration in pmol $\mathrm{gg}^{-1}$ of total protein $+\mathrm{SE}$ (left y-axis). Points above bars represent corresponding $\mathrm{F}_{\mathrm{v}} / \mathrm{F}_{\mathrm{M}}$ at each time point $\pm \mathrm{SE}$ (right $y$-axis). $X$-axis is time in hours from start of recovery conditions. Letters above bars indicate statistically distinct groups of D1 content within individual treatments as determined by Tukey's post hoc HSD tests $(\alpha=0.05, n=4)$.

\subsection{Aiptasia Experiment}

Changes in $\mathrm{F}_{\mathrm{V}} / \mathrm{F}_{\mathrm{M}}$ and $\mathrm{D} 1$ protein concentration relative to feeding regime (fed or starved) and temperature exposure (constant $26^{\circ} \mathrm{C}$ (low temperature) or increasing to $33^{\circ} \mathrm{C}$ (high temperature)) were examined in the symbiotic anemone Aiptasia, hosting either Symbiodiniaceae species Breviolum minutum or phylotype A4 or B2. 


\subsubsection{Influence of Feeding regime on growth of symbiotic Aiptasia}

Measurements of oral disc diameter of randomly selected Aiptasia showed a significant reduction in mean size of anemones that had been starved for six weeks $v s$. those that had been fed regularly. Anemones hosting phylotypes A4, B2 and B. minutum showed a mean decrease in size of $19 \%^{1}, 30 \%{ }^{2}$ and $34 \%{ }^{3}$ respectively at the end of the starvation period (Fig. 3.9 b). When compared to those anemones that had been fed regularly, starved anemones were on average $34 \%^{4}, 33 \%{ }^{5}$ and $38 \%{ }^{6}$ smaller when hosting phylotypes A4, B2 and B. minutum respectively (Fig 3.9 a-b). Only anemones hosting phylotype A4 showed a significant increase in size after six weeks of feeding $\left(13 \%{ }^{7}\right)$.

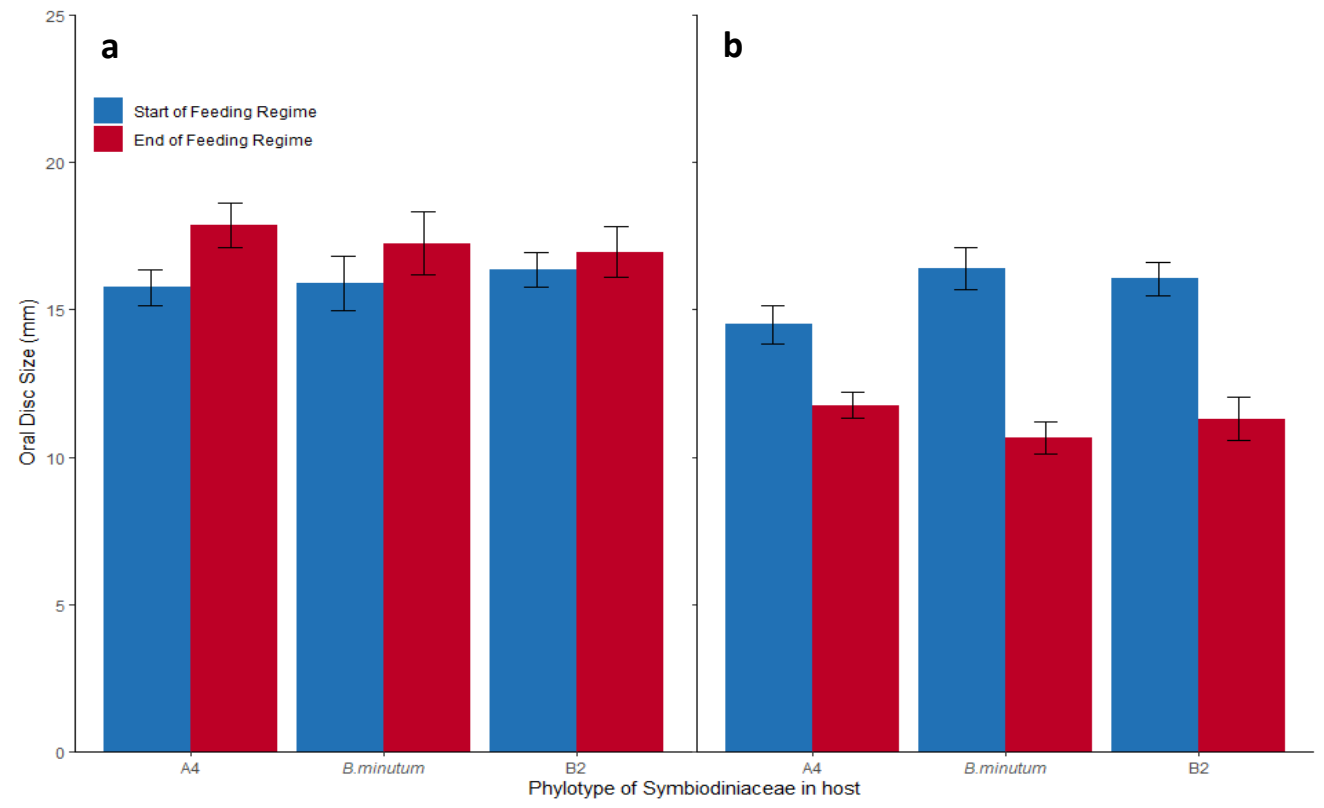

Fig. 3.9 Change in oral disc size of symbiotic Aiptasia due to feeding regime. a Aiptasia fed twice weekly for six weeks, b Aiptasia starved for six weeks. Means shown \pm SE

\subsubsection{Influence of temperature and host feeding regime on $F_{V} / F_{M}$ of Symbiodiniaceae in}

\section{Aiptasia}

Feeding regime of the host had only a minor influence on the $\mathrm{F}_{\mathrm{V}} / \mathrm{F}_{\mathrm{M}}$ values of in hospite (within the host) Symbiodiniaceae. Analogous to the culture experiment, the starved HT treatments of all phylotypes showed significant departure from their respective LT treatments earlier than the fed 
treatments; after $48 \mathrm{~h}$ at $33^{\circ} \mathrm{C}$ for B. minutum ${ }^{1}$ and after $72 \mathrm{~h}$ at $33^{\circ} \mathrm{C}$ for $\mathrm{A}^{2}$ and $\mathrm{B} 2^{3}$ (Fig. $3.10 \mathrm{~b}, \mathrm{~d}$, f). However, no significant difference was observed between fed and starved treatments of either temperature for phylotype A4 or B. minutum for more than 24 consecutive hours prior to recovery, or for more than two consecutive hours post recovery. For phylotype B2, a sustained significant difference was only observed between the high temperature treatments after entering recovery, with starved HT on average $88 \% \%^{4}$ that of fed HT from two hours post-recovery until the end of the recovery period 22 $\mathrm{h}$ later. Following $24 \mathrm{~h}$ recovery, the starved high temperature treatment of B. minutum was $25 \%{ }^{5}$ higher than A4, but not significantly different to B2, while the starved low temperature treatment of B2 was $18 \%^{6}$ and $21 \%{ }^{7}$ lower than $\mathrm{A} 4$ and B. minutum respectively.

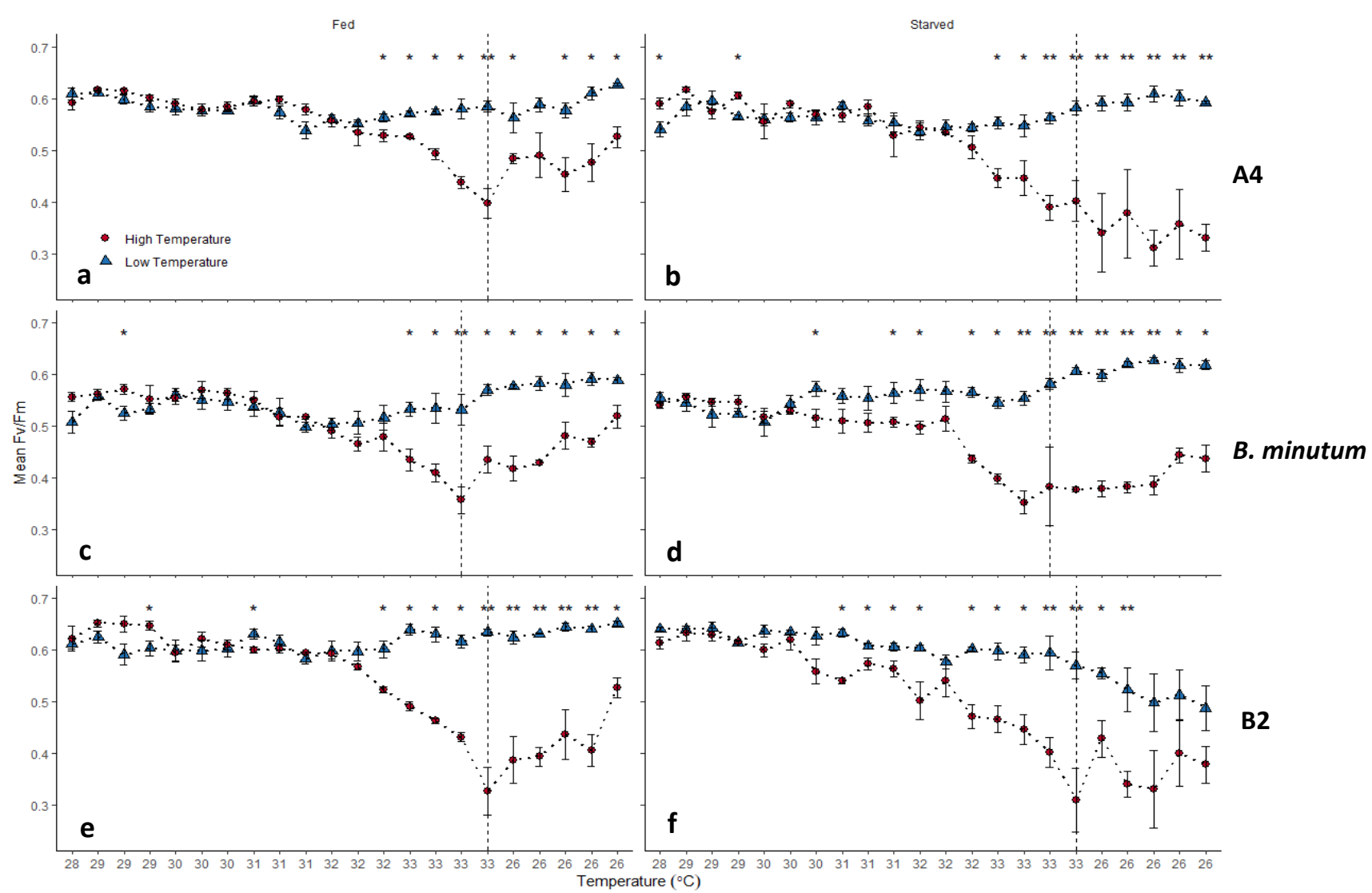

Fig 3.10 Changes in $\mathrm{F}_{\mathrm{v}} / \mathrm{F}_{\mathrm{M}}$ of Symbiodiniaceae as a response to temperature treatment in an Aiptasia host. Phylotypes A4 (ab), B. minutum (c-d) and B2 (e-f) hosted by anemones that had been either fed regularly for six weeks (left column) or starved for six weeks (right column). High temperature treatments (red circles) were increased from $26^{\circ} \mathrm{C}$ to $33^{\circ} \mathrm{C}$ by $1^{\circ} \mathrm{C}$ every three days, low temperature treatments (blue triangles) remained at $26^{\circ} \mathrm{C}$ throughout the experiment. Vertical dotted lines indicate start of recovery period $\left(26^{\circ} \mathrm{C}\right.$ and $20 \mu$ moles photons $\left.\mathrm{m}^{-2} \mathrm{~s}^{-1}\right)$. Prior to the start of recovery, tick marks on $\mathrm{y}$-axis represent periods of $24 \mathrm{~h}$. After entering recovery, tick marks represent $+2,+4,+6,+8$ and $+24 \mathrm{~h}$ from start of recovery respectively. (Note, anemones hosting $B$. minutum entered recovery $24 \mathrm{~h}$ earlier than phylotypes $\mathrm{A} 4$ and $\mathrm{B} 2$, so the final tick mark on the $\mathrm{y}$-axis represents $48 \mathrm{~h}$ after start of recovery in $\mathbf{c}$ and $\mathbf{d}$ only). Single asterisks above plots denote significant 
difference between temperature treatments (One-way ANOVA $p<0.05$ ), double asterisks denote a difference larger than $30 \%$. Means shown $\pm S E, n=4$.

\subsubsection{Influence of Temperature and Host Feeding regime on D1 protein concentration of}

\section{Symbiodiniaceae in Aiptasia}

*As the number of experimental organisms at the conclusion of the temperature treatment was severely limited (explained in Methods section 2.5.6), sampling of Aiptasia for protein extraction was restricted to four anemones per treatment per time-point. The concentration of D1 protein presented at each timepoint therefore reflects only the pooled D1 protein as a fraction of the total protein from each fouranemone sample, and cannot be used to infer statistical significance between treatment groups or timepoints. For this reason, the data were analysed in terms of fold-change, with a two-fold change in D1 protein concentration between samples being used as the minimum threshold beyond which any difference could be inferred. Accordingly, any trends and correlations relating to D1 concentration in Symbiodiniaceae in hospite are presented as putative, and cannot be considered to reflect the outcome of robust statistical testing.

Host feeding regime had a considerably larger influence on in hospite Symbiodiniaceae D1 protein concentration than it had on $\mathrm{F}_{\mathrm{V}} / \mathrm{F}_{\mathrm{M}}$ (Fig. 3.11), with the starved, high temperature treatments of all three phylotypes, and the starved, low temperature treatments of A4 and B2 showing only trace values of D1

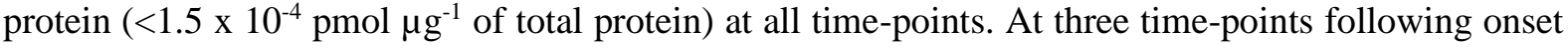
of recovery, D1 protein concentration in starved treatments of Aiptasia hosting phylotype B2 were below the level where they were distinguishable from background signal. The only starved treatment to show a net gain in D1 concentration was the low temperature treatment of B. minutum, which increased 2-fold over the $24 \mathrm{~h}$ recovery period.

The fed HT and LT treatments of A4 showed a $~ 3.2$ and 7.4-fold increase in D1 concentration respectively over $24 \mathrm{~h}$ of recovery, while fed LT of B. minutum and B2 increased $\sim 2.5$ and $\sim 2.4$-fold respectively from $24 \mathrm{~h}$ pre- to $24 \mathrm{~h}$ post-onset of recovery. 


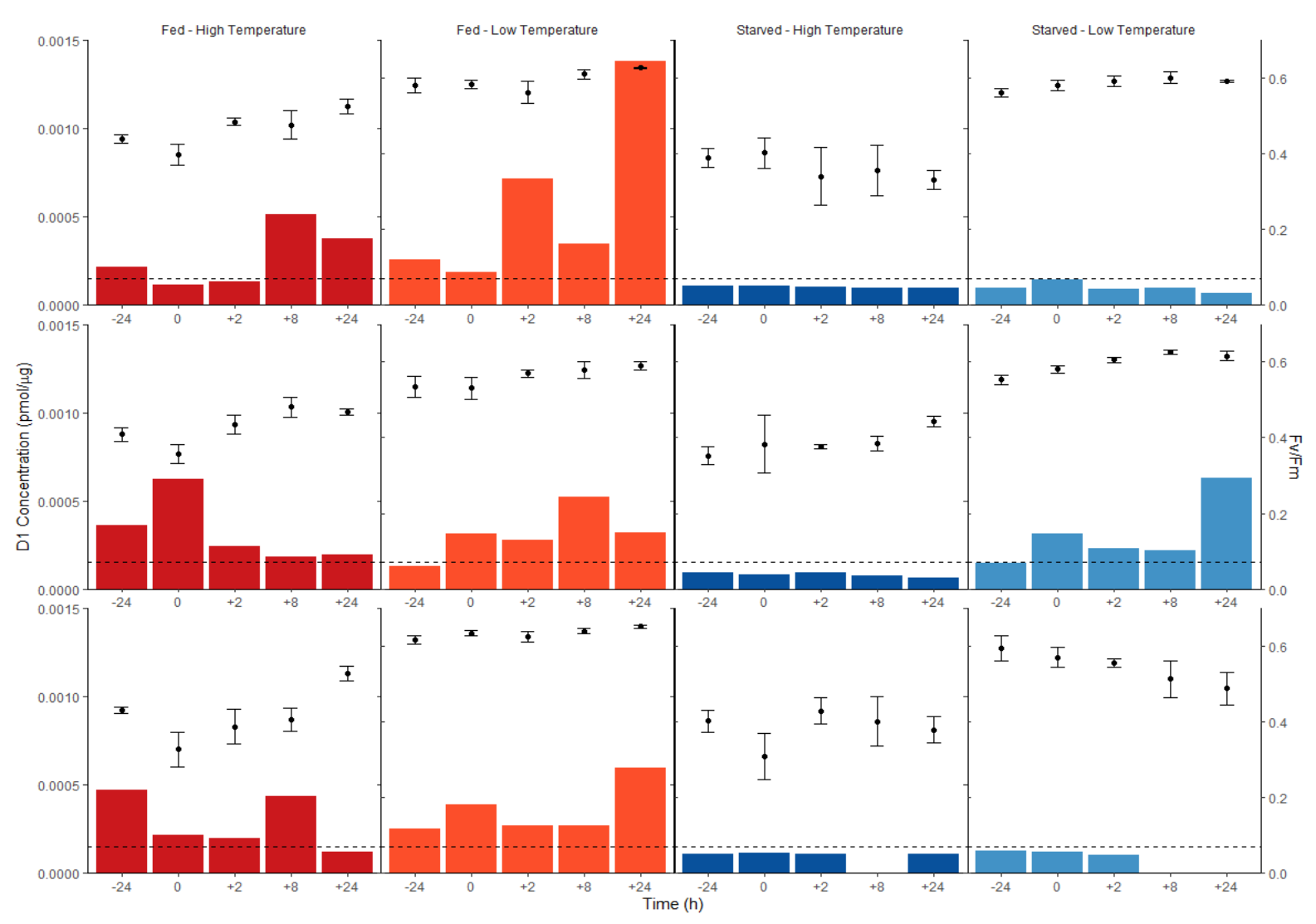

A4

B. minutum

Fig. 3.11 D1 protein content and corresponding $\mathrm{F}_{\mathrm{V}} / \mathrm{F}_{\mathrm{M}}$ of in hospite Symbiodiniaceae under different feeding regimes and temperature treatments. Aiptasia hosting phylotypes A4 (top row), B. minutum (middle row) and B2 (bottom row) either fed and exposed to high temperature $\left(33^{\circ} \mathrm{C}\right)$ (first column), fed and exposed to low temperature $\left(26^{\circ} \mathrm{C}\right)$ (second column), starved and exposed to high temperature (third column) or starved and exposed to low temperature (fourth column). Bars represent pooled D1 protein concentration of four anemones per time-point in pmol $\mu \mathrm{g}^{-1}$ of total protein (left $y$-axis). Points above bars represent corresponding mean $\mathrm{F}_{\mathrm{V}} / \mathrm{F}_{\mathrm{M}}$ at each time-point $\pm \mathrm{SE}$ (right $y$-axis). $\mathrm{X}$-axis is time in hours from start of recovery conditions. Bars stopping below dashed horizontal lines $\left(<1.5 \times 10^{-4} \mathrm{pmol} \mathrm{gg}^{-1}\right)$ have been extrapolated from polynomial models and are treated as trace concentrations. Missing bars indicate concentrations indistinguishable from background signal. 


\subsection{Appendix of statistical test parameters}

\begin{tabular}{|c|c|c|c|c|c|}
\hline 3.1. $\mathrm{F}_{\mathbf{V}} / \mathrm{F}_{\mathbf{M}}$ of Symbiodiniaceae Phylotype A4 \\
\hline$\#$ & Test & $\mathbf{d f}$ & F-value & P-value & Adjusted P-value \\
\hline 1 & ANOVA & 1 & 10.9 & 0.03 & - \\
\hline 2 & ANOVA & 1 & 29.9 & 0.005 & - \\
\hline 3 & ANOVA & 1 & 16.3 & 0.016 & - \\
\hline 4 & ANOVA & 1 & 26 & 0.007 & - \\
\hline 5 & ANOVA & 1 & 25.1 & 0.007 & - \\
\hline 6 & ANOVA & 1 & 21.7 & 0.009 & - \\
\hline 7 & ANOVA & 1 & 12.8 & 0.023 & - \\
\hline 8 & ANOVA & 1 & 69.8 & 0.001 & \\
\hline
\end{tabular}

\subsection{1. $F_{V} / F_{M}$ of Symbiodiniaceae Phylotype $A 4$}

\begin{tabular}{|c|c|c|c|c|c|}
\hline \multicolumn{6}{|c|}{ 3.2.1. $F_{V} / F_{M}$ of Symbiodiniaceae Phylotype A4 } \\
\hline$\#$ & Test & df & F-value & P-value & Adjusted P-value \\
\hline 1 & ANOVA & 1,62 & 26.5 & $<0.001$ & - \\
\hline 2 & ANOVA & 3,1212 & 455 & $<0.001$ & - \\
\hline \multicolumn{6}{|c|}{ 3.2.2 $F_{V} / F_{M}$ of Symbiodiniaceae Phylotype A4 } \\
\hline \# & Test & df & F-value & P-value & Adjusted P-value \\
\hline 1 & ANOVA & 1,14 & 16.74 & 0.001 & - \\
\hline 2 & ANOVA & 1,14 & 19.45 & $<0.001$ & - \\
\hline 3 & ANOVA & 1,14 & 32.5 & $<0.001$ & - \\
\hline 4 & ANOVA & 1,6 & 17.74 & 0.006 & - \\
\hline 5 & ANOVA & 1,6 & 9.65 & 0.021 & - \\
\hline 6 & ANOVA & 1,6 & 21.84 & 0.003 & - \\
\hline 7 & ANOVA & 1,6 & 57.11 & $<0.001$ & - \\
\hline 8 & KW-Dunn's & 3 & 8.65 & 0.003 & $0.02,0.02$ \\
\hline 9 & ANOVA & 1,6 & 13.99 & 0.009 & - \\
\hline 10 & ANOVA & 1,6 & 202.41 & $<0.001$ & - \\
\hline 11 & ANOVA & 1,206 & 283 & $<0.001$ & - \\
\hline 12 & ANOVA & 1,206 & 361 & $<0.001$ & - \\
\hline \multicolumn{6}{|c|}{ 3.2.3. $\quad F_{V} / F_{M}$ of Symbiodiniaceae Phylotype B2 } \\
\hline$\#$ & Test & df & F-value & P-value & Adjusted P-value \\
\hline 1 & ANOVA & 1,14 & 6.16 & 0.026 & - \\
\hline 2 & ANOVA & 1,14 & 26.86 & $<0.001$ & - \\
\hline 3 & ANOVA & 1,14 & 26.9 & $<0.001$ & - \\
\hline 4 & ANOVA & 1,14 & 13 & 0.003 & - \\
\hline 5 & ANOVA & 1,302 & 259 & $<0.001$ & - \\
\hline 6 & ANOVA & 1,6 & 18.4 & 0.005 & - \\
\hline 7 & ANOVA & 1,6 & 22.4 & 0.003 & - \\
\hline 8 & ANOVA & 1,14 & 62.7 & $<0.001$ & - \\
\hline 9 & ANOVA & 1,14 & 13.1 & 0.011 & - \\
\hline 10 & ANOVA & 1,6 & 16.9 & 0.006 & - \\
\hline 11 & ANOVA & 1,6 & 14.8 & 0.009 & - \\
\hline 12 & ANOVA & 1,158 & 151 & $<0.001$ & - \\
\hline 13 & ANOVA & 1,14 & 14.2 & 0.002 & - \\
\hline 14 & ANOVA/Tukey's HSD & 3,444 & 128 & $<0.001$ & $<0.001$ \\
\hline 15 & ANOVA/Tukey's HSD & 3,444 & 128 & $<0.001$ & $<0.001$ \\
\hline
\end{tabular}




\begin{tabular}{|c|c|c|c|c|c|}
\hline$\#$ & Test & df & F-value & P-value & Adjusted P-value \\
\hline 1 & ANOVA & 1,26 & 18.5 & $<0.001$ & - \\
\hline 2 & ANOVA & 1,26 & 79.3 & $<0.001$ & - \\
\hline 3 & ANOVA & 1,68 & 14 & $<0.001$ & - \\
\hline 4 & ANOVA & 1,7 & 14.5 & 0.007 & - \\
\hline 5 & ANOVA & 1,7 & 5.7 & 0.048 & - \\
\hline 6 & ANOVA & 1,88 & 29.4 & $<0.001$ & - \\
\hline 7 & ANOVA & 1,19 & 26 & $<0.001$ & - \\
\hline 8 & KW/Dunn's & 1,12 & 42.5 & $<0.001$ & 0.002 \\
\hline 9 & ANOVA & 1,68 & 58.7 & $<0.001$ & - \\
\hline \multicolumn{6}{|c|}{ 3.3.2 Fluorescence Parameter Curves of Phylotype B2 } \\
\hline \# & Test & df & F-value & P-value & Adjusted P-value \\
\hline 1 & ANOVA & 1,94 & 27.8 & $<0.001$ & - \\
\hline 2 & ANOVA & 1,10 & 5.17 & 0.046 & - \\
\hline 3 & ANOVA & 1,14 & 17.2 & $<0.001$ & - \\
\hline
\end{tabular}

\begin{tabular}{|c|c|c|c|c|c|}
\hline \multicolumn{2}{|c|}{ 3.4 Influence of Temperature and Nutrients on D1 Protein Concentration } \\
\hline$\#$ & Test & $\mathbf{d f}$ & F-value & P-value & Adjusted P-value \\
\hline 1 & ANOVA/Tukey's HSD & 3,12 & 10.5 & 0.001 & 0.002 \\
\hline 2 & ANOVA /Tukey's HSD & 3,12 & 7.46 & 0.004 & 0.017 \\
\hline 3 & ANOVA /Tukey's HSD & 3,12 & 5.06 & 0.019 & 0.032 \\
\hline 4 & ANOVA /Tukey's HSD & 3,12 & 7.98 & 0.003 & 0.043 \\
\hline 5 & ANOVA /Tukey's HSD & 3,12 & 3.85 & 0.039 & $<0.001$ \\
\hline 6 & ANOVA /Tukey's HSD & 3,12 & 10.6 & 0.001 & $<0.001$ \\
\hline 7 & ANOVA /Tukey's HSD & 3,12 & 4.3 & 0.028 & 0.034 \\
\hline 8 & ANOVA /Tukey's HSD & 3,12 & 4.86 & 0.019 & 0.029 \\
\hline 9 & ANOVA /Tukey's HSD & 3,12 & 20.3 & $<0.001$ & 0.007 \\
\hline 10 & ANOVA /Tukey's HSD & 3,12 & 7.64 & 0.004 & 0.008 \\
\hline 11 & ANOVA /Tukey's HSD & 3,12 & 17.7 & $<0.001$ & $<0.001$ \\
\hline 12 & ANOVA & 1,125 & 132 & $<0.001$ & - \\
\hline 13 & ANOVA & 1,124 & 104 & $<0.001$ & - \\
\hline
\end{tabular}

\begin{tabular}{|c|c|c|c|c|c|}
\hline 3.5.1 Influence of feeding regime on growth of symbiotic Aiptasia \\
\hline$\#$ & Test & df & F-value & P-value & Adjusted P-value \\
\hline 1 & ANOVA & 1,38 & 12.1 & 0.001 & - \\
\hline 2 & ANOVA & 1,38 & 26.4 & $<0.001$ & - \\
\hline 3 & ANOVA & 1,38 & 41.7 & $<0.001$ & $<0.001$ \\
\hline 4 & ANOVA/Tukey's HSD & 1,38 & 48.7 & $<0.001$ & $<0.001$ \\
\hline 5 & ANOVA/Tukey's HSD & 1,38 & 25.3 & $<0.001$ & $<0.001$ \\
\hline 6 & ANOVA/Tukey's HSD & 1,38 & 30.2 & $<0.001$ & \\
\hline 7 & ANOVA & 1,38 & 4.76 & 0.035 & \\
\hline
\end{tabular}

\begin{tabular}{|c|c|c|c|c|c|}
\hline \multicolumn{2}{|c|}{ 3.5.2 Influence of temperature and host feeding regime on $\mathrm{F}_{\mathrm{V}} / \mathrm{F}_{\mathbf{M}}$ of Symbiodiniaceae in Aiptasia } \\
\hline$\#$ & Test & $\mathbf{d f}$ & F-value & P-value & Adjusted P-value \\
\hline 1 & ANOVA & 1,6 & 6.71 & $<0.001$ & - \\
\hline 2 & ANOVA & 1,6 & 4.41 & $<0.001$ & - \\
\hline 3 & ANOVA & 1,6 & 19.63 & 0.004 & - \\
\hline 4 & ANOVA & 1,46 & 57.6 & $<0.001$ & 0.037 \\
\hline 5 & ANOVA/Tukey' HSD & 2,9 & 4.5 & 0.044 & 0.043 \\
\hline 6 & ANOVA/Tukey' HSD & 2,9 & 7.04 & 0.014 & 0.016 \\
\hline 7 & ANOVA/Tukey' HSD & 2,9 & 7.04 & 0.014 & - \\
\hline
\end{tabular}




\section{Discussion}

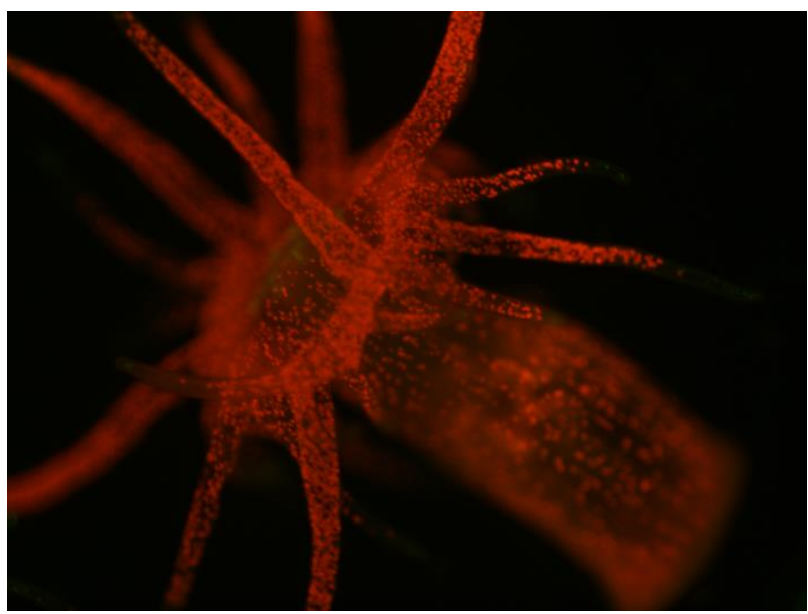

Aiptasia hosting Symbiodiniaceae phylotype A4

This thesis investigated the effects of nutrient availability on the thermotolerance of Symbiodiniaceae and as it relates to photosynthetic function. Measurements of photophysiological dynamics in cultured Symbiodiniaceae phylotypes A4 and B2 were used to describe the functional state of the algal chloroplast under experimentally manipulated nutrient and temperature conditions, giving insight into differences in nutrient allocation and photoprotective mechanisms employed by the algae under heat stress.

Symbiotic pairings of the model sea anemone Aiptasia with Symbiodiniaceae species B. minutum and phylotypes A4 and B2 were then examined to determine how the functional traits of the symbiont influence the fitness of the symbiotic partnership under varying nutrient and temperature conditions. The results of these studies are discussed below, with conclusions drawn about the functional physiology and adaptive history of the experimental organisms.

\subsection{Importance of nutrient availability for photosynthetic health of cultured}

\section{Symbiodiniaceae}

The ability to effectively utilize available nutrient resources, particularly nitrogen $(\mathrm{N})$ and phosphorous $(\mathrm{P})$, is of key concern when considering the photosynthetic potential of Symbiodiniaceae (Berges et al., 1996). Like most algae and higher plants, Symbiodiniaceae exhibit a high turnover of chloroplastic D1 
protein, even under moderate irradiance, as damaged D1 protein is degraded and synthesized de novo. This necessitates efficient use of $\mathrm{N}$ pools to maintain protein transcription and photochemical energy conversion, especially when in $\mathrm{N}$ depleted and/or high stress environments (Mattoo et al., 1984; Ohad et al., 1984; Aro et al., 1993; Keren et al., 1997; Liefer et al., 2019). The wide spectrum of sensitivity to thermally induced photoinhibition between species and genera of Symbiodiniaceae (Swain et al., 2017; Mansour et al., 2018) shows that photophysiological thermotolerance is genetic. Thus, if effective $\mathrm{N}$ utilization in plastid protein synthesis contributes to thermal tolerance, elevated concentrations of D1 protein should be common to thermally tolerant species/phylotypes in $\mathrm{N}$ depleted environments. This hypothesis was tested by comparing the photophysiology and rates of plastid protein synthesis between a thermally-tolerant (A4) and a thermally-sensitive (B2) phylotype of Symbiodiniaceae subjected to nutrient and temperature stress.

\subsubsection{Chloroplast protein synthesis and quantum yield}

In all cases, high nutrient availability was positively correlated with both maximum quantum yield $\left(\mathrm{F}_{\mathrm{V}} / \mathrm{F}_{\mathrm{M}}\right)$ and $\mathrm{D} 1$ protein concentration, showing that for both phylotypes low nutrient environments present a challenge to optimal photosynthetic ability. While this may at first appear obvious, it establishes that the efficiency of photosynthate production can be directly influenced by the algae's use of available nutrients. This has important consequences for host/symbiont relationships, as nutrient availability for in hospite Symbiodiniaceae is controlled by the host, and is typically $\mathrm{N}$ limited so as to regulate the rate of algal cell division (Yellowlees et al., 2008; Rädecker et al., 2015). Those algae that can maintain effective photosynthesis under such conditions would confer an obvious advantage to the host. What is less immediately clear however, is whether a direct correlation exists between D1 protein concentration and $\mathrm{F}_{\mathrm{V}} / \mathrm{F}_{\mathrm{M}}$. In the thermally-tolerant phylotype $\mathrm{A} 4$, recovery of $\mathrm{F}_{\mathrm{V}} / \mathrm{F}_{\mathrm{M}}$ after heat stress was accompanied by a corresponding increase in D1 protein, with overall concentrations positively correlated with nutrient availability. However, D1 concentrations in the thermally-sensitive B2 either decreased or remained stable during recovery, even while $\mathrm{F}_{\mathrm{V}} / \mathrm{F}_{\mathrm{M}}$ increased. Further to this, the $\mathrm{A} 4$ treatments had three times the D1 concentrations of B2, while exhibiting less than $10 \%$ difference in 
$\mathrm{F}_{\mathrm{V}} / \mathrm{F}_{\mathrm{M}}$ during recovery (Figs $3.7 \& 3.8$ ). While it appears that photosynthetic health and D1 protein concentration are positively correlated in the thermotolerant phylotype, considering the metabolic cost of protein synthesis, it seems anomalous that A4 should produce such an over-abundance of D1 protein, only to achieve comparable results to B2 in terms of photochemical potential.

Concentrations of D1 protein are directly linked with photoinactivation/reactivation, in a wide variety of photoautotrophs (Mattoo et al., 1981; Tyystjärvi et al., 1992; Nishiyama et al., 2004; Nath et al., 2013), and while there are many potential candidates for the molecular mechanism which initiates damage in photosystem II (PSII) (Hakala et al., 2004; Vass, 2012; Kulk et al., 2013) (and indeed there may be more than one mechanism depending on the environment/organism) it is well understood that damage to D1 is followed by its degradation and removal from PSII, followed by synthesis de novo of a new D1 molecule and reinsertion into the PSII reaction centre (RCII) (Nath et al., 2013). During the process of degradation, removal and reinsertion of the D1 protein, the photosystem is considered to be inactivated as the functional and structural integrity of the RC is directly reliant on the presence of D1. Whether D1 degradation or re-synthesis is the rate limiting factor in this process is a topic of ongoing research, and may also be dependent on the environment and organism (Takahashi et al., 2004; Jimbo et al., 2018), but typically, lower concentrations of D1 at any one time equate to lower numbers of functional RC's, and thus lower photosynthetic potential, and vice versa.

How then does this equate to two organisms with considerably different D1 concentrations showing comparable photosynthetic potential? An explanation may be related to differences in the physiology of the two phylotypes A4 and B2, particularly with regard to adaptive traits in various subunits of the thylakoid membrane. These points will be addressed in the following sections.

\subsubsection{Evidence for physiological disparity between $\mathrm{A} 4$ and $\mathrm{B} 2$ in response to light exposure}

The results of the dark-light (D-L) induction curves (Figs $3.5 \& 3.6$ ) showed that the two phylotypes react in singular and concise ways when exposed to equal levels of irradiance. Phylotype A4 consistently initiated a strong non-photochemical quenching (NPQ) response within the first minute of exposure, which gradually decreased over time, whereas B2 reacted in an opposing manner, gradually 
increasing its NPQ response over time. The form of NPQ initiated in these short light exposure periods $(<10 \mathrm{~min})$ is termed energy quenching $(q E) \mathrm{NPQ}$, and is directly related to acidification of the thylakoid lumen in the chloroplast. Briefly, protons (hydrogen ions, $\mathrm{H}^{+}$) are pumped from the oxygen evolving complex (OEC, found in the reaction centre of PSII) into the thylakoid lumen upon initiation of photochemical conversion, contributing to a $\mathrm{pH}$ gradient between the lumen and the stroma (Ruban, 2016). This intra-thylakoid $\Delta \mathrm{pH}$ is used to synthesize ATP via the protein ATP synthase, primarily for use in carbon fixation in the Calvin-Benson cycle (CBC). If the $\mathrm{CBC}$ is not able to consume the ATP at the same rate at which it's synthesized, the $\Delta \mathrm{pH}$ will continue to build, initiating de-epoxidation of the LHC xanthophyll pigment diadinoxanthin (Dd) to diatoxanthin (Dt) (Hill et al., 2012). This deepoxidation leads to aggregation of LHC pigments, causing photon energy to be converted to heat energy $(q E)$ before it reaches RCII, alleviating excitation pressure on PSII and the downstream electron transport chain (ETC) (Sacharz et al., 2017; Morris \& Fleming, 2018). The rate and duration of this process depends on various factors, including irradiance levels, ambient temperature and the functional state of the thylakoid proteins and stromal metabolites (Moejes et al., 2017). Thus, under controlled conditions, the disparity in $q E$ initiation between the two phylotypes may indicate potential physiological differences in various areas of their respective photosynthetic apparatus.

\subsubsection{Physiological profile of phylotype A4}

The initial steep rise in NPQ in all treatments of phylotype A4 indicates a rapid drop in the luminal pH at the start of the actinic period, almost certainly due to a fast build-up of ATP in the stroma. This could conceivably be caused by a delay in turnover of the CBC, owing to a lack of oxidised NADP available for reduction at the terminal ferrodoxin-NADP reductase (FNR). However, in this particular case this scenario is unlikely, as the 10 minute dark adaptation period prior to measurements should have allowed for ample re-oxidation of NADPH to NADP (Cardol et al., 2011). An alternative explanation concerns the number of reaction centres contributing to the luminal proton pool. In a scenario where phylotype A4 had a high concentration of functioning RCII's (equivalent to high concentrations of D1 protein), the onset of light exposure would cause a rapid and substantial drop in luminal $\mathrm{pH}$ as the RC's start pumping protons into the lumen in unison. This would correspond with a rapid increase in NPQ, after 
which proton ingress slows considerably and luminal $\mathrm{pH}$ begins to rise as ATP synthesis draws protons out of the lumen (Goss \& Lepetit, 2015). As ATP is consumed by the CBC and more ATP is synthesized, the luminal $\mathrm{pH}$ will continue to increase and NPQ will begin to ease. From here, a feedback loop of increasing luminal $\mathrm{pH}$ followed by easing of NPQ would result in an eventual equilibrium where proton ingress matches egress and photochemical conversion becomes optimal (Papageorgiou \& Govindjee, 2004).

\subsubsection{Profile of high nutrient A4}

Applying the above process to the D-L induction curve of the high nutrient-low temperature treatment of A4 (Fig. 3.5a), we see this feed-back loop resulting in virtually no NPQ at the end of the actinic period and an effective quantum yield (Y(II)) more or less equal to the maximum quantum yield $\left(\mathrm{F}_{\mathrm{V}} / \mathrm{F}_{\mathrm{M}}\right.$, the first $\mathrm{Y}$ (II) point on the $\mathrm{x}$-axis). This shows that under optimal conditions Symbiodiniaceae of phylotype A4 are able to process all incoming light photochemically, indicating a high number of functioning RC's (as reflected in the high D1 content) and efficient use of downstream metabolic products in the $\mathrm{CBC}$.

Under high-nutrient, high temperature conditions (Fig. 3.5c) the same initial rise in NPQ is followed by a more gradual decline, reaching approximately $50 \%$ of the initial value after 10 minutes, which is indicative of increased ATP synthesis due to cyclic electron transport (CET) (Arnon et al., 1954; Shikanai, 2014). CET involves the recycling of electrons from the terminal ferrodoxin (Fd) in PSI to the electron acceptor plastoquinone (PQ), which transports protons back into the thylakoid lumen via cytochrome b6f, increasing net ATP synthesis (Allen, 2003). CET is complimentary to electron transport via PSII (linear electron transport, LET), and is necessary to balance proton movement and ATP synthesis under ambient conditions. CET up-regulates in some Symbiodiniaceae types (and in particular in the genus Symbiodinium, to which phylotype A4 belongs (McCabe Reynolds et al., 2008)) as a stress response to elevated temperatures (Aihara et al., 2016; Dang et al., 2019b), increasing $q E$ to further protect the photosystems from photoinactivation.

Despite this sustained NPQ, D1 concentration in the high temperature treatment was less than $50 \%$ that of the low temperature treatment prior to entering recovery, which can be attributed to a lower rate of 
protein synthesis rather than a higher rate of protein degradation, and is linked to changes in carbon fixation efficiency in the CBC at increased temperatures (Brooks \& Farquhar, 1985). The enzyme ribulose-1,5-bisphosphate carboxylase/oxygenase (Rubisco) which catalyses fixation of $\mathrm{CO}_{2}$ (carboxylation) in the $\mathrm{CBC}$, also has a (slightly) lower affinity for $\mathrm{O}_{2}$ fixation (oxygenation) at a rate of approximately 4:1 $\quad \mathrm{CO}_{2} / \mathrm{O}_{2}$, under atmospheric conditions and at moderate ambient temperatures $\left(\sim 25^{\circ} \mathrm{C}\right)($ Ogren, 1984$)$. As ambient temperature increases past $30^{\circ} \mathrm{C}$, the specificity of Rubisco for $\mathrm{CO}_{2}$ drops considerably, resulting in increased oxygenation relative to carboxylation and leading to altered pools of metabolites within the $\mathrm{CBC}$. This causes a slowing of the rate of carbon fixation in the high temperature treatments and a subsequent backlog of the reducing agent, NADPH. Takahashi and Murata (2006) showed that synthesis of glycerate-3-phosphate (3-PGA) through carboxylation is essential for facilitating linear electron flow through the ETC by utilizing NADPH. When carboxylation becomes limited due to increased oxygenation, excess electrons are diverted away from NADPH production to an alternative electron sink known as the water - water cycle (WWC) in PSI (Curien et al., 2016). Here, the excess electrons reduce $\mathrm{O}_{2}$ to the reactive oxygen species (ROS) superoxide $\left(\mathrm{O}_{2}{ }^{-}\right)$via the Mehler reaction (Mehler, 1951). Under ambient conditions, $\mathrm{O}_{2}{ }^{-}$is then rapidly converted to the $\mathrm{ROS}_{2} \mathrm{O}_{2}$ via superoxide dismutase (SOD) and then to water via ascorbate peroxidase (APX), in a series of reactions collectively known as the Mehler Ascorbate Peroxidase (MAP) pathway (Asada, 2006; Roberty et al., 2014). It is proposed that when electron flow through the WWC produces ROS at a rate beyond which SOD and APX can effectively scavenge it, a build-up of ROS can spread from PSI to interfere with various plastid and cellular processes (Roberty et al., 2015; Warner \& Suggett, 2016). In particular $\mathrm{H}_{2} \mathrm{O}_{2}$ interferes with transcription of the psbA gene encoding for D1, by oxidising the transcription factor EfTu (Takahashi \& Murata, 2008; Jimbo et al., 2018), involved in the elongation step of psbA mRNA. Thus, lower concentrations of D1 protein observed under heat stress in the high nutrient treatments of A4 were likely a result of slowed protein synthesis due to accumulation of ROS, a supposition reinforced by the rapid increase in D1 protein upon transfer to low temperature. 


\subsubsection{Profile of low nutrient A4}

Both of the low nutrient treatments of $A 4$ had consistently lower $F_{V} / F_{M}$ than the high nutrient treatments (Figs 3.2a-b \& 3.4a-c). This is reflected in the relatively high initial $\mathrm{Y}(\mathrm{NO})$ of the low nutrient D-L induction curves (Fig. 3.5a \& b), indicating that a larger proportion of the RC pool is inactive in the low nutrient treatment, which is also consistent with the relatively low observed D1 concentrations. It then follows that when this smaller pool of active RC's initiate NPQ, a larger proportion of xanthophylls become de-epoxidised relative to the high nutrient treatments, leaving a net lower number of available RC's to continue photochemical conversion, seen as decreased Y(II) throughout the actinic period. The more gradual decline in NPQ of the low nutrient treatments compared to high, could be an indication of limitations imposed on the CBC due to low nitrogen (N) availability. Alipanah et al. (2015) found that NADPH production through photosynthesis was reduced in the diatom Phaeodactylum tricornutum when grown in $\mathrm{N}$ depleted medium, with downregulation of multiple gene transcripts involved in photosynthesis, including transcripts for FNR, for enzymes used in the CBC and for biophysical carbon concentrating mechanisms. A large decrease in carbon-fixation capacity in nitrogen starved microalgae (Rhodomonas sp., Cryptophyceae)(da Silva et al., 2009) was attributed to lowered stores of rubisco, with similar phenomena observed in other species of microalgae and cyanobacteria (Berges et al., 1996; Kulk et al., 2013, 2018; Liefer et al., 2018; Coulombier et al., 2020). Thus, a similar outcome to temperature stress with regards to NPQ is observed under low N availability, where ATP synthesis outpaces consumption and $\Delta \mathrm{pH}$ remains elevated, prolonging NPQ throughout the actinic period. The down-regulation of CBC activity could also have contributed to a decreased rate of D1 synthesis via ROS transcription interference, however the effect appears to be minimal at low temperature, likely due to reduced pressure on the ETC as a result of a smaller pool of active RCs.

The result of heat stress and low nutrients combined (Fig. 3.5d) presents an accumulative effect of the aforementioned physiological responses, characterised by sustained NPQ and little recovery of Y(II) throughout the actinic period. $\mathrm{Y}(\mathrm{NO})$ under these conditions is significantly higher than in any of the other treatments, indicating that photoinhibition has occurred in a substantial portion of PSII reaction centres, from which the algae were not able to recover. Again, this is reflected in low D1 concentration 
relative to non-heat stressed treatments (Fig.3.7 e \& f vs. Fig.3.7 g \& h) and is most likely a consequence of extended periods of low carbon fixation due to nitrogen depleted conditions, exacerbated by decreased carboxylation efficiency at elevated temperature.

\subsubsection{Physiological profile of phylotype B2}

Phylotype B2 showed a marked contrast in NPQ initiation relative to phylotype A4, indicating distinct differences in the physiological mechanisms employed in the photosynthetic apparatus. Whereas the luminal $\mathrm{pH}$ of phylotype $\mathrm{A} 4$ dropped rapidly and substantially at the onset of actinic light before increasing at a rate dependent on the treatment conditions, luminal $\mathrm{pH}$ of phylotype $\mathrm{B} 2$ consistently decreased over time and with less variability than A4 (Fig. $3.6 \&$ Table 3.1). This immediately suggests that phylotype B2 has a comparatively smaller pool of active RCII's contributing to acidification of the thylakoid lumen, a conclusion supported by the low D1 protein concentration of all B2 treatment groups. This may also be supported by the relatively flat curve of the Y(II) parameter compared to Y(II) and $\mathrm{Y}(\mathrm{NO})$, which can be elucidated as follows.

At the onset of actinic light, we see a dip in $\mathrm{Y}(\mathrm{II})$ with a corresponding peak in $\mathrm{Y}(\mathrm{NO})$ and a moderate rise in $\mathrm{Y}(\mathrm{NPQ})$. The peak in $\mathrm{Y}(\mathrm{NO})$ represents the active RC's which have transiently closed due to reduction of electron acceptors downstream of PSII, while the rise in Y(NPQ) represents RC's which are no longer receiving excitation energy from the LHC due to initiation of $q E$ (Minagawa, 2013). At the next saturation pulse (SP), Y(II) increases by slightly less than the amount that $\mathrm{Y}(\mathrm{NO})$ decreases, indicating reopening of the transiently closed RC's as electron flow becomes steady through the ETC, coupled with a small rise in $\mathrm{Y}(\mathrm{NPQ})$ as more protons are pumped into the lumen. For the remaining SP's up until the end of actinic exposure, Y(II) remains almost flat, while a slow increase in Y(NPQ) is mirrored by a decrease in $\mathrm{Y}(\mathrm{NO})$. This may indicate that from this point on, initiation of $q E$ no longer reroutes excitation energy away from active RC's, but instead initiates de-epoxidation of xanthophylls in areas of the antenna complex where there is no direct connection between the LHC and an active PSII, resulting in a decrease in steady state fluorescence $\left(\mathrm{F}_{\mathrm{s}}\right)$ through the quenching of chlorophyll $a$ (Chl-a) molecules bound to inactive reaction centres (Alexandre et al., 2007). This proposed uncoupling 
of $q E$ from RC inactivation would assume an antenna pigment complex following the 'lake model' (Kramer et al., 2004), where PSII reaction centres share a common pigment bed and excitation energy can be passed between multiple LHC's. In this scenario, when PSII density is low and interconnectivity between antenna pigments is high, an increase in the ratio of inactive to active RC's (equivalent to a decrease in the effective quantum yield, $Y($ II $)$ ) would logically increase the rate at which incoming excitation energy is passed to an inactive RC. The LHC of Symbiodiniaceae is unusual among light harvesting organisms in that it utilizes a water soluble peridinin-chlorophyll-a-protein (PCP) with a high carotenoid/chlorophyll ratio unique to dinoflagellates (Boldt et al., 2012; Maruyama et al., 2015). The PCP has been shown to greatly facilitate quenching of triplet state chlorophyll- $a\left({ }^{3} \mathrm{Chl}-\right.$ $\left.a^{*}\right)$ (Alexandre et al., 2007), a product of intersystem crossing in singlet state Chl- $a\left({ }^{1} \mathrm{Chl}-a^{*}\right)$ formed via charge recombination in inactivated RC's (Ballottari et al., 2013). Thus the LHC itself may potentially act as a photoprotective mechanism, quenching excess energy absorbed into the antenna complex without the need for interaction with a functional RC.

For the above explanation to be plausible, phylotype B2 would need to possess a very large antenna complex relative to PSII, with active and inactive RCs sharing excitation energy. The size of the antenna complex relative to PSII is known as the effective absorption cross-section ( $\left.\sigma_{\mathrm{PSII}}\right)$ (Suggett et al., 2010). Low-light adapted photoautotrophs generally present an enlarged $\sigma_{\text {PSII }}$, with increased numbers of light harvesting pigments and more numerous connections between neighbouring LHCs relative to high-light adapted organisms (Hennige et al., 2009). This increase in $\sigma_{\text {PSII }}$ effectively casts a 'wider photon net', allowing the chloroplast to absorb maximal light in steady, low-light environments using a relatively small number of active RCs. Conversely, an organism with a smaller $\sigma_{P S I I}$ and a higher number of active $\mathrm{RCs}$ is more efficient under conditions of transient or high irradiance, where maximal photoconversion can be achieved quickly, and rapid onset of $q E$ effectively alleviates excess exciton pressure by directly disengaging RC's from the ETC (McCabe Reynolds et al., 2008).

An increased $\sigma_{\mathrm{PSII}}$ in phylotype B2 would explain why no significant difference was observed in $\mathrm{Y}(\mathrm{NPQ})$ between any of the treatments, as changes in luminal $\mathrm{pH}$ would be held more or less constant by the bottleneck created by a large LHC:PSII ratio. The slightly lower Y(II) observed in the low nutrient treatments compared to their high nutrient counterparts may then simply be a consequence of 
limited D1 protein replacement due to low $\mathrm{N}$ availability. Further research to fully elucidate the effects of antennae pigment size on NPQ initiation may involve comparisons of NPQ response and $\sigma_{\text {PSII }}$ between Symbiodiniaceae acclimated to different light levels. The potential for PCP to act as a quenching mechanism independent of PSII functionality is also a concept which could have a large impact on our current understanding of the photophysiological responses of Symbiodiniaceae. So far, research into this topic has been limited to studies of isolated pigment complexes only, so methodologies involving in vivo study of this important topic are much needed.

\subsubsection{Relationship between thylakoid composition and D1 protein concentration}

The disparity in D1 protein concentration between the two phylotypes can be explained via the ratio of active to inactive PSII's in their respective thylakoids. By definition, the fluorescence parameter termed maximum quantum yield $\left(\mathrm{F}_{\mathrm{V}} / \mathrm{F}_{\mathrm{M}}\right)$ reflects the number of photosynthetically viable $\mathrm{RCs}$ in a sample as a proportion of all RC's in that sample. It then follows that two samples with an unequal number of active $\mathrm{RCs}$, can in fact show a very similar $\mathrm{F}_{\mathrm{V}} / \mathrm{F}_{\mathrm{M}}$, so long as the proportion of active to inactive $\mathrm{RCs}$ is the same in both. Park et al., (1996) showed that photoinactivation of functional PSII is intrinsic and independent of antenna size, and that under moderate light a roughly equal number of RCs will be undergoing degradation, removal and reinsertion of D1 regardless of the acclimation status of the organism. This explains how phylotype A4 can have such a high D1 concentration relative to B2 while showing a comparable $\mathrm{F}_{\mathrm{V}} / \mathrm{F}_{\mathrm{M}}$ value; the proportion of inactive RCs is similar in each phylotype, regardless of the total number of RCs.

The thylakoid of phylotype A4 likely contains a high density of active RCs, as indicated by a rapid NPQ response and high D1 content, which would suggest that it is adapted for high light environments characterised by fluctuating irradiance, where quick responses to changes in irradiance are necessary for optimizing photochemistry. This would be consistent with a symbiont preferentially associated with corals found in shallow areas of the reef, where rapidly fluctuating, high irradiance is expected (McCabe Reynolds et al., 2008). Conversely, the thylakoid of phylotype B2 is characterised by low RC density, as indicated by a slow NPQ response and low D1 concentration, likely as an adaptation to low light 
environments where irradiance remains relatively stable, ie. deeper areas of the reef (Suggett et al., 2015a).

In terms of the effect of nutrient availability on photophysiology, we saw a consistently higher photosynthetic yield in A4 than in B2 when under high nutrient conditions, suggesting an emphasis in A4 on metabolic processes favouring production of PSIIs with rapid turnover of D1 protein when $\mathrm{N}$ is abundantly available. However, this trend was not seen under low nutrient availability, where B2 was generally more photsynthetically viable. This may suggest a somewhat opportunistic use of nutrients in A4 compared to B2, again reflecting the particular habitat phylotype A4 may be adapted to. Shallow areas of the reef are characterised by warm, highly stratified water, where nutrients arrive in irregular pulses carried by wind-induced vertical-mixing of deeper, more nutrient-rich water (Canuto, 2004). Under these conditions, opportunistic, rapid use of nutrients as soon as they become available may be to phylotype A4's advantage. On the other hand, if phylotype B2 preferentially inhabits corals in deeper water, where access to nutrients is more uniform, adaptations resulting in slower, more steady use of nutrients may lower metabolic costs to both the symbiont and the host (Cunning \& Baker, 2013), as discussed in the next section.

These conclusions agree with work by Suggett et al., (2015), using measurements of $\sigma_{\text {PSII }}$ and NPQ responses in 18 Symbiodiniaceae phylotypes to determine phylogenetic trends in photobiological characteristics. Here photosynthetic variability was suggested to correlate with functional diversity resulting from differences in microhabitat and adaptations to changing irradiance. McCabe Reynolds $e t$ al., (2008) came to similar conclusions regarding photophysiological adaptations in genus Symbiodinium and Breviolum (the genera of A4 and B2 respectively), showing a connection between differences in light harvesting mechanisms in the symbiont and depth of the host (see also IglesiasPrieto et al., 2004). 


\subsection{Influence of host nutritional status on photosynthetic health of symbiotic}

\section{Symbiodiniaceae}

The cnidarian hosts of Symbiodiniaceae are typically mixotrophic, with the bulk of their daily energy needs provided by algal symbionts in the form of carbohydrates and fatty acids (photosynthates) (Muscatine \& Cernichiari, 1969; Papina et al., 2003), supplemented by heterotrophic ingestion of inorganic nitrogen and organic carbon from zooplankton and particulate organic matter (Rahav et al., 1989; McAuley \& Cook, 1994). Metabolic by-products from the host, particularly ammonium $\left(\mathrm{NH}_{4}{ }^{+}\right)$ and $\mathrm{CO}_{2}$, are in turn consumed by Symbiodiniaceae to maintain growth and photosynthesis (Rädecker et al., 2015). Efficient uptake and recycling of nutrients between the host and the symbiont is therefore crucial to maintaining a mutualistic symbiosis from which both partners benefit.

Importantly, nutrients provided to the symbiont via the host must be regulated to ensure that the endosymbiont population remains at a density conducive to effective photosynthesis and photosynthate translocation, as higher symbiont densities are associated with lower chlorophyll production and decreased fixed carbon translocation from the symbiont to the host (Hoogenboom et al., 2010; Wall et al., 2020), and may even contribute to coral bleaching (Cunning \& Baker, 2013). Nitrogen limitation is a key strategy employed by the host to stabilize symbiont growth rates (Rädecker et al., 2015; Xiang et al., 2020), leading to the hypothesis that Symbiodiniaceae able to optimize photosynthesis in low nitrogen environments would confer maximal benefits to the host in terms of growth-rate and resilience to environmental stress. This was tested by subjecting Aiptasia hosting Symbiodiniaceae species $B$. minutum and phylotypes A4 and B2 to nutrient and heat stress, while examining changes in host growthrate and the photophysiological response of the symbionts.

\subsubsection{Influence of symbiosis on photosynthetic potential}

Differences in $\mathrm{F}_{\mathrm{V}} / \mathrm{F}_{\mathrm{M}}$ between fed and starved treatments were minimal in the low temperature treatments of A4 and B. minutum, as well as between the fed treatments of all phylotypes. This contrasts with observations of cultured Symbiodiniaceae, where $\mathrm{F}_{\mathrm{V}} / \mathrm{F}_{\mathrm{M}}$ values in high nutrient treatments were significantly elevated over low nutrient treatments for the majority of the experiment, and $\mathrm{F}_{\mathrm{V}} / \mathrm{F}_{\mathrm{M}}$ of high 
nutrient A4 was at all times higher than that of B2. This is potentially due to active dissolved inorganic carbon (DIC) concentration by the host, leading to improved carbon fixation efficiency by the symbiont. Barott et al., (2015) showed that cnidarian hosts expend ATP to pump $\mathrm{H}^{+}$into the symbiosome, the vacuole inhabited by the symbiont within the gastrodermal cells of the host, in a process akin to reverse ATP synthesis (Müller et al., 1996). This leads to acidification of the symbiosome, promoting conversion of bicarbonate ions $\left(\mathrm{HCO}_{3}^{-}\right)$to $\mathrm{CO}_{2}$ in the presence of carbonic anhydrases (Weis, 1993). The resulting increase in $\mathrm{CO}_{2}$ concentration greatly improves carboxylation efficiency during algal photosynthesis (Kaplan et al., 1980), increasing the rate of photosynthetic output and likely reducing the need for alternate electron sinks, in particular the WWC, as NADPH consumption in the CBC increases. Decreased electron flow to the WWC leads to lower rates of $\mathrm{H}_{2} \mathrm{O}_{2}$ production (Asada, 1999), improving efficiency of psbA-D1 transcription. By extension, this may also explain the apparently increased thermotolerance of $\mathrm{A} 4$ and $\mathrm{B} 2$ in hospite vs. in culture, as loss of $\mathrm{CO}_{2}$ specificity in Rubisco at elevated temperature is mediated by increased $\mathrm{CO}_{2}$ concentration in the chloroplast stroma (Hartman \& Harpel, 1994).

\subsubsection{Influence of Symbiodiniaceae type on host growth}

Oral disc size between Aiptasia hosting different phylotypes was remarkably uniform for both feeding regimes, indicating that no one phylotype confers any particular benefit to host growth-rate as a result of nutrient availability. This agrees with the conclusions regarding $\mathrm{F}_{\mathrm{V}} / \mathrm{F}_{\mathrm{M}}$ from above, where it appears all three phylotypes are equally capable of maximising photosynthetic potential, and thus photosynthate translocation, when benefitting from the host mediated environment at moderate temperature.

\subsubsection{Influence of feeding and heat stress in hospite on D1 protein concentration}

Concentrations of D1 protein in the fed treatments also showed a similar outcome, with no significant difference observed between phylotypes. Again, in terms of D1 synthesis, it appears that nutrient utilization in all three phylotypes was more or less equal regardless of temperature, as long as the host was well supplied with food. This was not the case in the starved, high temperature treatments however, 
where D1 concentration was extremely low in all cases, indicating that the symbionts were severely limited in $\mathrm{N}$. This could either be due to an increased rate of D1 turnover as a result of heat stress in the symbiont or, more likely, decreased access to nutrients translocated from the host. This may highlight the importance of host heterotrophy when dealing with thermal stress. It is well understood that access to particulate organic matter (POM) is essential to corals recovering from bleaching, as heterotrophy becomes the only means for the coral to acquire fixed carbon and $\mathrm{N}$ after expulsion of symbionts (Edmunds \& Davies, 1986; Grottoli et al., 2006). But evidence suggests that corals with increased rates of heterotrophy during symbiosis are more resilient to heat induced bleaching, regardless of the thermotolerance of the associated Symbiodiniaceae (Conti-Jerpe et al., 2020). Under short-term starvation, hosts are thought to support symbiont cell division and growth through a supply of nutrients from their own stored energy reserves (eg. lipids, proteins, carbohydrates) (Grottoli et al., 2006; Grottoli \& Rodrigues, 2011), hence it may be the case that increased demands on host metabolism resulting from heat stress in the host caused a shift in nutrient availability away from the symbiont in favour of the host (Borell et al., 2008). This may have important consequences for corals on highly oligotrophic reefs, where low nutrient availability limits heterotrophy, potentially increasing the susceptibility of corals to heat induced bleaching.

Interestingly, an increase in D1 protein concentration in the starved, low temperature treatments was seen only in Aiptasia hosting B. minutum. B. minutum is a homologous symbiont to Aiptasia, in that it is most commonly found in Aiptasia in the wild. This would suggest that the symbiotic partnership between these two organisms is relatively well optimized compared to other Symbiodiniaceae types. However phylotypes A4 and B2 are also found in wild Aiptasia but less commonly, and it is clear that different symbiotic partnerships can confer different benefits depending on the environment (Parkinson \& Baums, 2014; Hoadley et al., 2019). It would appear that under conditions of starvation, the symbiosis between Aiptasia and B. minutum allows for a continuous supply of nutrients to the symbiont, as evidenced by ongoing D1 synthesis, which may be related to the higher growth rate of $B$. minutum relative to phylotypes A4 and B2 (Wooldridge, 2013). Symbionts with a high mitotic index (MI) place an increased metabolic demand on their hosts compared to those with low MI, especially at low population densities, when fixed carbon translocation to the host is at a minimum (Baghdasarian \& 
Muscatine, 2000; Xiang et al., 2020). In cnidarians hosting multiple Symbiodiniaceae types, it has been shown that those symbionts with the higher MI are more likely to be expelled by the host under conditions of stress (McCloskey et al., 1996). While the MI of the three phylotypes involved in these experiments was not measured, it may be that $B$. minutum was able to continue synthesizing D1 at low nutrient levels due to its energy demands for growth being less significant at low population densities. Thus, while efficient nutrient utilization is clearly important in the current instance, the health of the host may not be directly linked to photosynthetic metabolism.

\subsection{Conclusion}

The cnidarian-dinoflagellate symbiosis is a complex and potentially fragile partnership, but it is clear that physiological diversity within symbionts and hosts allows for a wide range of responses to changing environmental and trophic conditions. The thermotolerant Symbiodiniaceae phylotype used in this study showed an increased rate of chloroplastic protein synthesis relative to the thermally sensitive phylotype when nutrient replete, but a decreased rate when nutrient limited. Therefore, the efficiency of nutrient use in photosynthetic pathways does not appear to be the limiting factor in thermotolerance. Rather, it was shown that patterns of nutrient utilization and photosynthetic productivity were likely a consequence of adaptations to specific environments, suggesting that thermal sensitivity may be governed by a more complex set of physiological variables. Investigations into adaptations in the photosynthetic complex, specifically resistance against heat-induced limitation of the CBC and advanced mechanisms of photoprotection (ie. Chl-a triplet quenching by antenna pigments) may provide key insights into the thermotolerance of certain Symbiodiniaceae.

The efficiency of the symbiont in utilizing available nutrients in photosynthetic pathways does not appear to have a simple linear correlation with the health or thermotolerance of the host. Partner specificity is likely to influence the ability of the host to withstand certain external stressors, and it is probable that sub-optimal partnerships contribute to internal stressors when environmental conditions become less favourable. However, efficient use of nutrient pools by the symbiont in pathways other than photosynthesis (ie. growth) when nutrients are lacking, may assist in reducing metabolic demands 
on the host and help the symbiosis endure periods of external stress. Further work on the influence of growth patterns in Symbiodiniaceae and its effects on host metabolism are needed in this respect.

Coral bleaching due to anthropogenic climate change is an immediate and global issue, one which has the potential to devastate some of the world's most diverse ecosystems. Research into the mechanisms facilitating the cnidarian-dinoflagellate symbiosis is critical for our understanding of how the world's reefs will be affected over the coming decades, and how we can act to protect this unique and fragile habitat. 


\section{References}

Abrego, D., Van Oppen, M. J. H. \& Willis, B. L. (2009). Highly infectious symbiont dominates initial uptake in coral juveniles. Molecular Ecology, 18(16), 3518-3531.

https://doi.org/10.1111/j.1365-294X.2009.04275.x

Aihara, Y., Takahashi, S. \& Minagawa, J. (2016). Heat induction of cyclic electron flow around photosystem i in the symbiotic dinoflagellate Symbiodinium. Plant Physiology, 171(1), 522529. https://doi.org/10.1104/pp.15.01886

Aldridge, G. M., Podrebarac, D. M., Greenough, W. T. \& Weiler, I. J. (2008). The use of total protein stains as loading controls: An alternative to high-abundance single-protein controls in semiquantitative immunoblotting. Journal of Neuroscience Methods, 172(2), 250-254. https://doi.org/10.1016/j.jneumeth.2008.05.003

Alexandre, M. T. A., Lührs, D. C., Van Stokkum, I. H. M., Hiller, R., Groot, M. L., Kennis, J. T. M. \& Van Grondelle, R. (2007). Triplet state dynamics in peridinin-chlorophyll-a-protein: A new pathway of photoprotection in LHCs? Biophysical Journal, 93(6), 2118-2128. https://doi.org/10.1529/biophysj.107.106674

Alipanah, L., Rohloff, J., Winge, P., Bones, A. M. \& Brembu, T. (2015). Whole-cell response to nitrogen deprivation in the diatom Phaeodactylum tricornutum. Journal of Experimental Botany, 66(20), 6281-6296. https://doi.org/10.1093/jxb/erv340

Allakhverdiev, S. I. \& Murata, N. (2004). Environmental stress inhibits the synthesis de novo of proteins involved in the photodamage-repair cycle of Photosystem II in Synechocystis sp. PCC 6803. Biochimica et Biophysica Acta - Bioenergetics, 1657(1), 23-32. https://doi.org/10.1016/j.bbabio.2004.03.003

Allakhverdiev, S. I., Nishiyama, Y., Takahashi, S., Miyairi, S., Suzuki, I. \& Murata, N. (2005). Systematic Analysis of the Relation of Electron Transport and ATP Synthesis to the Photodamage and Repair of Photosystem II in Synechocystis. Physiology, 137(1), 263-273. https://doi.org/10.1104/pp.104.054478

Allen, J. F. (2003). Cyclic, pseudocyclic and noncyclic photophosphorylation: New links in the chain. Trends in Plant Science, 8(1), 15-19. https://doi.org/10.1016/S1360-1385(02)00006-7

Anderson, J. M., Park, Y.-I. \& Chow, W. S. (1998). Unifying model for the photoinactivation of Photosystem II in vivo under steady-state photosynthesis. Photosynthesis Research (Vol. 56).

Arnon, D. I., Allen, M. B. \& Whatley, F. R. (1954). Photosynthesis by isolated chloroplasts. Nature, 174(4426), 394-396. https://doi.org/10.1038/174394a0

Aro, E. M., McCaffery, S. \& Anderson, J. M. (1993). Photoinhibition and D1 protein degradation in peas acclimated to different growth irradiances. Plant Physiology, 103(3), 835-843. https://doi.org/10.1104/pp.103.3.835

Asada, K. (1999). THE WATER-WATER CYCLE IN CHLOROPLASTS: Scavenging of Active Oxygens and Dissipation of Excess Photons. Annu. Rev. Plant Physiol. Plant Mol. Biol (Vol. 50). Retrieved from www.annualreviews.org

Asada, K. (2006). Production and Scavenging of Reactive Oxygen Species in Chloroplasts and Their Functions. Plant Physiology (Vol. 141).

Bachtiar, I., Ghafari, M. I. A., Rahman, I., Hilda, B. \& Mahrus. (2019). Coral Echinoporalamellosa hosts multiple clades of symbionts in Western Alas Strait, Indonesia. AIP Conference Proceedings, 2199(December), 070013. https://doi.org/10.1063/1.5141327 
Baghdasarian, G. \& Muscatine, L. (2000). Preferential expulsion of dividing algal cells as a mechanism for regulating algal-cnidarian symbiosis. Biological Bulletin.

https://doi.org/10.2307/1543184

Baird, A. H., Guest, J. R. \& Willis, B. L. (2009). Systematic and biogeographical patterns in the reproductive biology. Annual Review of Ecology, Evolution, and Systematics, 40(2009), 551571. https://doi.org/10.1146/annurev.ecolsys.1

Baker, A. C. (2003). Flexibility and Specificity in Coral-Algal Symbiosis: Diversity, Ecology, and Biogeography of Symbiodinium. Annual Review of Ecology, Evolution, and Systematics, 34(1), 661-689. https://doi.org/10.1146/annurev.ecolsys.34.011802.132417

Baker, A. C., Glynn, P. W. \& Riegl, B. (2008). Climate change and coral reef bleaching: An ecological assessment of long-term impacts, recovery trends and future outlook. Estuarine, Coastal and Shelf Science, 80, 435-471. https://doi.org/10.1016/j.ecss.2008.09.003

Baker, D. M., Freeman, C. J., Wong, J. C. Y., Fogel, M. L. \& Knowlton, N. (2018). Climate change promotes parasitism in a coral symbiosis. The ISME Journal, 12, 921-930. https://doi.org/10.1038/s41396-018-0046-8

Ballottari, M., Mozzo, M., Girardon, J., Hienerwadel, R. \& Bassi, R. (2013). Chlorophyll triplet quenching and photoprotection in the higher plant monomeric antenna protein Lhcb5. Journal of Physical Chemistry B, 117(38), 11337-11348. https://doi.org/10.1021/jp402977y

Barott, K. L., Venn, A. A., Perez, S. O., Tambutté, S. \& Tresguerres, M. (2015). Coral host cells acidify symbiotic algal microenvironment to promote photosynthesis. Proceedings of the National Academy of Sciences, 112(2). https://doi.org/10.1073/pnas.1413483112

Bassham, A., Calvin, M. \& Benson, A. (1950). The Path of Carbon in Photosynthesis. J. Biol. Chem., (2).

Berges, J. A., Charlebois, D. O., Mauzerall, D. C., Falkowski, P. G., Berges, J. A., Charlebois, D., ... B, N. Y. J. A. (1996). Differential Effects of Nitrogen Limitation on Photosynthetic Efficiency of Photosystems I and II in Microalgae. American Society of Plant Biologists, 110(2), 689-696.

Berry, J. \& Bjorkman, O. (1980). Photosynthetic Response and Adaptation to Temperature in Higher Plants. Annual Review of Plant Physiology, 31(1), 491-543. https://doi.org/10.1146/annurev.pp.31.060180.002423

Bhagooli, R. \& Hidaka, M. (2004). Photoinhibition, bleaching susceptibility and mortality in two scleractinian corals, Platygyra ryukyuensis and Stylophora pistillata, in response to thermal and light stresses. Comparative Biochemistry and Physiology Part A, 137, 547-555. https://doi.org/10.1016/j.cbpb.2003.11.008

Bhuyan, A. K. (2010). On the mechanism of SDS-induced protein denaturation. Biopolymers, 93(2), 186-199. https://doi.org/10.1002/bip.21318

Blank, R. J. \& Trench, R. K. (1985). Speciation and symbiotic dinoflagellates. Science, 229(4714), 656-658. https://doi.org/10.1126/science.229.4714.656

Boldt, L., Yellowlees, D. \& Leggat, W. (2012). Hyperdiversity of Genes Encoding Integral LightHarvesting Proteins in the Dinoflagellate Symbiodinium sp. PLOS ONE, 7(10), e47456-e47456. https://doi.org/10.1371/journal.pone.0047456

Borell, E. M., Yuliantri, A. R., Bischof, K. \& Richter, C. (2008). The effect of heterotrophy on photosynthesis and tissue composition of two scleractinian corals under elevated temperature. Journal of Experimental Marine Biology and Ecology, 364(2), 116-123. https://doi.org/10.1016/j.jembe.2008.07.033

Boussac, A. \& Rutherford, A. W. (1992). The involvement of Ca 2+ in the Ca2+-effect on 
Photosystem-II oxygen evolution. Photosynthesis Research (Vol. 32).

Bricker, T. M. \& Frankel, L. K. (2002). The structure and function of CP47 and CP43 in Photosystem II. Photosynthesis Research (Vol. 72).

Brooks, A. \& Farquhar, G. D. (1985). Effect of temperature on the CO2/O2 specificity of ribulose1,5-bisphosphate carboxylase/oxygenase and the rate of respiration in the light - Estimates from gas-exchange measurements on spinach. Planta, 165(3), 397-406. https://doi.org/10.1007/BF00392238

Bumann, D. \& Oesterhelt, D. (1995). Destruction of a Single Chlorophyll is Correlated with the Photoinhibition of Photosystem II with a Transiently Inactive Donor Side. Source (Vol. 92).

Canuto, V. M. (2004). Latitude-dependent vertical mixing and the tropical thermocline in a global OGCM. Geophysical Research Letters, 31(16), L16305. https://doi.org/10.1029/2004GL019891

Carballo-Bolaños, R., Soto, D. \& Chen, C. A. (2019). Thermal Stress and Resilience of Corals in a Climate-Changing World. Journal of Marine Science and Engineering, 8(1), 15. https://doi.org/10.3390/jmse8010015

Cardol, P., Forti, G. \& Finazzi, G. (2011). Regulation of electron transport in microalgae. Biochimica et Biophysica Acta - Bioenergetics, 1807(8), 912-918. https://doi.org/10.1016/j.bbabio.2010.12.004

Carlos, A. \& Baillie, B. (1999). Phylogenetic Position of Symbiodinium (Dinophyceae) Isolates From Tridacnids (Bivalvia), Cardiids (Bivalvia), a Sponge (Porifera), a. Journal of Phycology, 35, 1054-1062. Retrieved from http://onlinelibrary.wiley.com/doi/10.1046/j.15298817.1999.3551054.x/abstract

Carpenter, K. E., Abrar, M., Aeby, G., Aronson, R. B., Banks, S., Bruckner, A., ... Wood, E. (2008). One-third of reef-building corals face elevated extinction risk from climate change and local impacts. Science, 321(5888), 560-563. https://doi.org/10.1126/science.1159196

Chow, W. S. \& Aro, E.-M. (2006). Photoinactivation and Mechanisms of Recovery. In Photosystem II (pp. 627-648). Springer-Verlag. https://doi.org/10.1007/1-4020-4254-x_28

Coffroth, M. A., Poland, D. M., Petrou, E. L., Brazeau, D. A. \& Holmberg, J. C. (2010). Environmental symbiont acquisition may not be the solution to warming seas for reef-building corals. PLoS ONE, 5(10). https://doi.org/10.1371/journal.pone.0013258

Conti-Jerpe, I. E., Thompson, P. D., Wong, C. W. M., Oliveira, N. L., Duprey, N. N., Moynihan, M. A. \& Baker, D. M. (2020). Trophic strategy and bleaching resistance in reef-building corals. Science Advances, 6(15), 5443-5453. https://doi.org/10.1126/sciadv.aaz5443

Cook, C. B., Muller-parker, G., Elia, C. F. D. \& Jan, N. (1992). Ammonium Enhancement of Dark Carbon Fixation and Nitrogen Limitation in Symbiotic Zooxanthellae : Effects of Feeding and Starvation of the Sea Anemone Aiptasia pallida Ammonium enhancement of dark carbon fixation and nitrogen limitation in symbiotic zooxa. Limnology, 37(1), 131-139.

Costanza, R. \& D'Arge, R. (1997). The value of the world's ecosystem services and natural capital. Nature, 387(6630), 253-260. Retrieved from https://www-naturecom.ezproxy.royalroads.ca/articles/387253a0.pdf

Coulombier, N., Nicolau, E., Le Déan, L., Barthelemy, V., Schreiber, N., Brun, P., ... Jauffrais, T. (2020). Effects of Nitrogen Availability on the Antioxidant Activity and Carotenoid Content of the Microalgae Nephroselmis sp. Marine Drugs, 18(9), 453.

https://doi.org/10.3390/md18090453

Croce, R., Morosinotto, T. \& Bassi, R. (2007). LHCI: The Antenna Complex of Photosystem I in Plants and Green Algae. In Photosystem I (pp. 119-137). Springer Netherlands. 
https://doi.org/10.1007/978-1-4020-4256-0_10

Cui, G., Liew, Y. J., Li, Y., Kharbatia, N., Zahran, N. I., Emwas, A. H., ... Aranda, M. (2019). Hostdependent nitrogen recycling as a mechanism of symbiont control in Aiptasia. PLoS Genetics, 15(6), e1008189-e1008189. https://doi.org/10.1371/journal.pgen.1008189

Cunning, R. \& Baker, A. C. (2013). Excess algal symbionts increase the susceptibility of reef corals to bleaching. Nature Climate Change, 3(3), 259-262. https://doi.org/10.1038/nclimate1711

Curien, G., Flori, S., Villanova, V., Magneschi, L., Giustini, C., Forti, G., ... Finazzi, G. (2016). The water to water cycles in microalgae. Plant and Cell Physiology. https://doi.org/10.1093/pcp/pcw048

da Silva, A. F., Lourenço, S. O. \& Chaloub, R. M. (2009). Effects of nitrogen starvation on the photosynthetic physiology of a tropical marine microalga Rhodomonas sp. (Cryptophyceae). Aquatic Botany, 91(4), 291-297. https://doi.org/10.1016/j.aquabot.2009.08.001

Dang, K. Van, Pierangelini, M., Roberty, S. \& Cardol, P. (2019a). Alternative Photosynthetic Electron Transfers and Bleaching Phenotypes Upon Acute Heat Stress in Symbiodinium and Breviolum spp. (Symbiodiniaceae) in Culture. Frontiers in Marine Science, 6, 656. https://doi.org/10.3389/fmars.2019.00656

Dang, K. Van, Pierangelini, M., Roberty, S. \& Cardol, P. (2019b). Alternative Photosynthetic Electron Transfers and Bleaching Phenotypes Upon Acute Heat Stress in Symbiodinium and Breviolum spp. (Symbiodiniaceae) in Culture. Frontiers in Marine Science, 6, 656. https://doi.org/10.3389/fmars.2019.00656

Das, P., Nutan, K. K., Singla-Pareek, S. L. \& Pareek, A. (2015). Oxidative environment and redox homeostasis in plants: Dissecting out significant contribution of major cellular organelles. Frontiers in Environmental Science, 2(JAN), 70. https://doi.org/10.3389/fenvs.2014.00070

Davy, S. K., Allemand, D. \& Weis, V. M. (2012). Cell Biology of Cnidarian-Dinoflagellate Symbiosis. Microbiology and Molecular Biology Reviews, 76(2), 229-261. https://doi.org/10.1128/mmbr.05014-11

Davy, Simon K \& Cook, C. B. (2001). The influence of "host release factor" on carbon release by zooxanthellae isolated from fed and starved Aiptasia pallida (Verrill). Comparative Biochemistry and Physiology, 129, 487-494.

De Las Rivas, J., Andersson, B. \& Barber, J. (1992). Two sites of primary degradation of the D1protein induced by acceptor or donor side photo-inhibition in photosystem II core complexes. FEBS Letters, 301(3), 246-252. https://doi.org/10.1016/0014-5793(92)80250-K

Degasperi, A., Birtwistle, M. R., Volinsky, N., Rauch, J., Kolch, W. \& Kholodenko, B. N. (2014). Evaluating strategies to normalise biological replicates of western blot data. PLoS ONE, 9(1). https://doi.org/10.1371/journal.pone.0087293

Demmig-Adams, B. \& Adams, W. W. (2006). Photoprotection in an ecological context: The remarkable complexity of thermal energy dissipation. New Phytologist, 172(1), 11-21. https://doi.org/10.1111/j.1469-8137.2006.01835.x

Díaz-Almeyda, E. M., Prada, C., Ohdera, A. H., Moran, H., Civitello, D. J., Iglesias-Prieto, R., ... Medina, M. (2017). Intraspecific and interspecific variation in thermotolerance and photoacclimation in Symbiodinium dinoflagellates. Proceedings of the Royal Society B: Biological Sciences, 284(1868). https://doi.org/10.1098/rspb.2017.1767

Diffenbaugh, N. S., Singh, D., Mankin, J. S., Horton, D. E., Swain, D. L., Touma, D., ... Rajaratnam, B. (2017). Quantifying the influence of global warming on unprecedented extreme climate events. Proceedings of the National Academy of Sciences of the United States of America, 114(19), 4881-4886. https://doi.org/10.1073/pnas.1618082114 
Douglas, A. E. (2008). Conflict, Cheats and the Persistence of Symbioses. Source: The New Phytologist, 177(4), 849-858. https://doi.org/10.1111/j.1469-8137.2007.02326.x

Eaton, S. L., Roche, S. L., Llavero Hurtado, M., Oldknow, K. J., Farquharson, C., Gillingwater, T. H. \& Wishart, T. M. (2013). Total Protein Analysis as a Reliable Loading Control for Quantitative Fluorescent Western Blotting. PLoS ONE, 8(8), e 72457. https://doi.org/10.1371/journal.pone.0072457

Edmunds, P. J. \& Davies, P. S. (1986). An energy budget for Porites porites (Scleractinia). Marine Biology: International Journal on Life in Oceans and Coastal Waters, 92(3), 339-347. https://doi.org/10.1007/BF00392674

Fabina, N. S., Putnam, H. M., Franklin, E. C., Stat, M. \& Gates, R. D. (2012). Transmission Mode Predicts Specificity and Interaction Patterns in Coral-Symbiodinium Networks. PLoS ONE, 7(9), 1-9. https://doi.org/10.1371/journal.pone.0044970

Ferrier-Pagè, C., Ezzat, L. L. L., Maguer, J.-F., Grover, R. \& Ferrier-Pagès, C. (2015). New insights into carbon acquisition and exchanges within the coral-dinoflagellate symbiosis under $\mathrm{NH} 4 \mathrm{p}$ and NO 32 supply. Proceedings of the Royal Society B: Biological Sciences. https://doi.org/10.1098/rspb.2015.0610

Finney, J. C., Pettay, D. T., Sampayo, E. M., Warner, M. E., Oxenford, H. A. \& LaJeunesse, T. C. (2010). The relative significance of host-habitat, depth, and geography on the ecology, endemism, and speciation of coral endosymbionts in the genus Symbiodinium. Microbial Ecology, 60(1), 250-263. https://doi.org/10.1007/s00248-010-9681-y

Gallagher, S. R. (2012). One-Dimensional SDS Gel Electrophoresis of Proteins. Current Protocols in Protein Science, 68(1), 10.1.1-10.1.44. https://doi.org/10.1002/0471140864.ps1001s68

Ganot, P., Moya, A., Magnone, V., Allemand, D., Furla, P. \& Sabourault, C. (2011). Adaptations to endosymbiosis in a Cnidarian-Dinoflagellate association: Differential gene expression and specific gene duplications. PLoS Genetics, 7(7). https://doi.org/10.1371/journal.pgen.1002187

Gassmann, M., Grenacher, B., Rohde, B. \& Vogel, J. (2009). Quantifying Western blots: Pitfalls of densitometry. Electrophoresis, 30(11), 1845-1855. https://doi.org/10.1002/elps.200800720

Gibbin, E. M., Krueger, T., Putnam, H. M., Barott, K. L., Bodin, J., Gates, R. D. \& Meibom, A. (2018). Short-term thermal acclimation modifies the metabolic condition of the coral holobiont. Frontiers in Marine Science, 5(FEB). https://doi.org/10.3389/fmars.2018.00010

Gilbert, S. F., Sapp, J. \& Tauber, A. I. (2012). A symbiotic view of life: we have never been individuals. The Quarterly Review of Biology, 87(4), 325-41. Retrieved from http://www.ncbi.nlm.nih.gov/pubmed/23397797

Glynn, P. W. (1993). Coral reef bleaching: ecological perspectives. Coral Reefs, 12(1), 1-17. https://doi.org/10.1007/BF00303779

Goss, R. \& Lepetit, B. (2015). Biodiversity of NPQ. Journal of Plant Physiology, 172, 13-32. https://doi.org/10.1016/j.jplph.2014.03.004

Grant, A. J., Rémond, M., People, J. \& Hinde, R. (1997). Effects of host-tissue homogenate of the scleractinian coral Plesiastrea versipora on glycerol metabolism in isolated symbiotic dinoflagellates. Marine Biology, 128(4), 665-670. https://doi.org/10.1007/s002270050133

Grottoli, A. G. \& Rodrigues, L. J. (2011). Bleached Porites compressa and Montipora capitata corals catabolize $\delta 13 \mathrm{C}$-enriched lipids. Coral Reefs, 30(3), 687-692. https://doi.org/10.1007/s00338011-0756-0

Grottoli, A. G., Rodrigues, L. J. \& Palardy, J. E. (2006). Heterotrophic plasticity and resilience in bleached corals. Nature, 440(7088), 1186-1189. https://doi.org/10.1038/nature04565 
Haan, C. \& Behrmann, I. (2007). A cost effective non-commercial ECL-solution for Western blot detections yielding strong signals and low background. Journal of Immunological Methods, 318(1-2), 11-19. https://doi.org/10.1016/j.jim.2006.07.027

Hakala, M., Tuominen, I., Keränen, M., Tyystjärvi, T. \& Tyystjärvi, E. (2004). Evidence for the role of the oxygen-evolving manganese complex in photoinhibition of Photosystem II. Biochimica et Biophysica Acta - Bioenergetics, 1706(1-2), 68-80. https://doi.org/10.1016/j.bbabio.2004.09.001

Hartman, F. C. \& Harpel, M. R. (1994). STRUCTURE, FUNCTION, REGULATION, AND ASSEMBLY OF D-RIBULOSE-1,5-BISPHOSPHATE CARBOXYLASE/OXYGENASE. Annual Review of Biochemistry, 63, 197-234.

Hennige, S. J., Suggett, D. J., Warner, M. E., McDougall, K. E. \& Smith, D. J. (2009). Photobiology of Symbiodinium revisited: Bio-physical and bio-optical signatures. Coral Reefs, 28(1), 179195. https://doi.org/10.1007/s00338-008-0444-x

Hill, R., Larkum, A. W. D., Prášil, O., Kramer, D. M., Szabó, M., Kumar, V. \& Ralph, P. J. (2012). Light-induced dissociation of antenna complexes in the symbionts of scleractinian corals correlates with sensitivity to coral bleaching. Coral Reefs, 31(4), 963-975. https://doi.org/10.1007/s00338-012-0914-z

Hill, R. \& Takahashi, S. (2014). Photosystem II recovery in the presence and absence of chloroplast protein repair in the symbionts of corals exposed to bleaching conditions. Coral Reefs, 33(4), 1101-1111. https://doi.org/10.1007/s00338-014-1188-4

Hill, R, Larkum, A., Frankart, C., Kühl, M. \& Ralph, P. (2004). Loss of Functional Photosystem II Reaction Centres in Zooxanthellae of Corals Exposed to Bleaching \{...\}. Photosynthesis Research, 59-72. Retrieved from http://www.springerlink.com/index/JG2R761368063858.pdf

Hill, Ross, Brown, C. M., DeZeeuw, K., Campbell, D. A. \& Ralph, P. J. (2011). Increased rate of D1 repair in coral symbionts during bleaching is insufficient to counter accelerated photoinactivation. Limnology and Oceanography, 56(1), 139-146. https://doi.org/10.4319/lo.2011.56.1.0139

Hill, Ross, Frankart, C. \& Ralph, P. J. (2005). Impact of bleaching conditions on the components of non-photochemical quenching in the zooxanthellae of a coral. Journal of Experimental Marine Biology and Ecology, 322(1), 83-92. https://doi.org/10.1016/j.jembe.2005.02.011

Hill, Ross \& Ralph, P. J. (2005). Diel and seasonal changes in fluorescence rise kinetics of three scleractinian corals. Functional Plant Biology, 32(6), 549-559. https://doi.org/10.1071/FP05017

Hill, Ross, Szabó, M., Rehman, A. U., Vass, I., Ralph, P. J. \& Larkum, A. W. D. (2014). Inhibition of photosynthetic $\mathrm{CO} 2$ fixation in the coral Pocillopora damicornis and its relationship to thermal bleaching. Journal of Experimental Biology, 217(12), 2150-2162.

https://doi.org/10.1242/jeb.100578

Hoadley, K. D., Lewis, A. M., Wham, D. C., Pettay, D. T., Grasso, C., Smith, R., ... Warner, M. E. (2019). Host-symbiont combinations dictate the photo-physiological response of reef-building corals to thermal stress. Scientific Reports, 9(1), 1-15. https://doi.org/10.1038/s41598-019$46412-4$

Hoogenboom, M., Beraud, E. \& Ferrier-Pagès, C. (2010). Relationship between symbiont density and photosynthetic carbon acquisition in the temperate coral Cladocora caespitosa. Coral Reefs, 29(1), 21-29. https://doi.org/10.1007/s00338-009-0558-9

Hughes, T. P., Barnes, M. L., Bellwood, D. R., Cinner, J. E., Cumming, G. S., Jackson, J. B. C., ... Scheffer, M. (2017). Coral reefs in the Anthropocene. Nature, 546(7656), 82-90. https://doi.org/10.1038/nature22901 
Hume, B. C. C. C., D'angelo, C., Smith, E. G., Stevens, J. R., Burt, J. \& Wiedenmann, J. (2015). Symbiodinium thermophilum sp. nov., a thermotolerant symbiotic alga prevalent in corals of the world's hottest sea, the Persian/Arabian Gulf. Scientific Reports, 5, 1-9. https://doi.org/10.1038/srep08562

Iglesias-Prieto, R., Beltrán, V. H., LaJeunesse, T. C., Reyes-Bonilla, H. \& Thomé, P. E. (2004). Different algal symbionts explain the vertical distribution of dominant reef corals in the eastern Pacific. Proceedings of the Royal Society B: Biological Sciences, 271(1549), 1757-1763. https://doi.org/10.1098/rspb.2004.2757

Iglesias-Prieto, Roberto, Matta, J. L., Robins, W. A. \& Trench, R. K. (1992). Photosynthetic Response to Elevated Temperature in the Symbiotic Dinoflagellate Symbiodinium microadriaticum in Culture. Proceedings of the National Academy of Sciences of the United States of America, 89(21), 10302-10305.

Innis, T., Cunning, R., Ritson-Williams, R., Wall, C. B. \& Gates, R. D. (2018). Coral color and depth drive symbiosis ecology of Montipora capitata in Kāne'ohe Bay, O'ahu, Hawai'i. Coral Reefs, 37(2), 423-430. https://doi.org/10.1007/s00338-018-1667-0

Ishikita, H., Saenger, W., Biesiadka, J., Loll, B. \& Knapp, E.-W. (2006). How Photosynthetic Reaction Centers Control Oxidation Power in Chlorophyll Pairs P680, P700, and P870. PNAS, 103(26), 9855-9860. https://doi.org/10.1073/pnas.0601446103

Jackson, J., Donovan, M., Cramer, K. \& Lam, V. (2012). Status and Trends of Caribbean Coral Reefs. Global Coral Reef Monitoring Network, IUCN, Gland, Switzerland.

Jahns, P. \& Holzwarth, A. R. (2012). The role of the xanthophyll cycle and of lutein in photoprotection of photosystem II. Biochimica et Biophysica Acta - Bioenergetics, 1817(1), 182-193. https://doi.org/10.1016/j.bbabio.2011.04.012

Janes, K. A. (2015). An analysis of critical factors for quantitative immunoblotting. Science Signaling, 8(371), rs2-rs2. https://doi.org/10.1126/scisignal.2005966

Jeans, J., Szabó, M., Campbell, D. A., Larkum, A. W. D., Ralph, P. J. \& Hill, R. (2014). Thermal bleaching induced changes in photosystem II function not reflected by changes in photosystem II protein content of Stylophora pistillata. Coral Reefs, 33(1), 131-139. https://doi.org/10.1007/s00338-013-1091-4

Jeong, H. J., Lee, S. Y., Kang, N. S., Yoo, Y. Du, Lim, A. S., Lee, M. J., ... Lajeunesse, T. C. (2014). Genetics and morphology characterize the dinoflagellate Symbiodinium voratum, n. sp., (dinophyceae) as the Sole representative of Symbiodinium clade e. Journal of Eukaryotic Microbiology, 61(1), 75-94. https://doi.org/10.1111/jeu.12088

Jimbo, H., Yutthanasirikul, R., Nagano, T., Hisabori, T., Hihara, Y. \& Nishiyama, Y. (2018). Oxidation of translation factor EF-Tu inhibits the repair of photosystem II. Plant Physiology, 176(4), 2691-2699. https://doi.org/10.1104/pp.18.00037

Jones, A. M., Berkelmans, R., Van Oppen, M. J. H., Mieog, J. C. \& Sinclair, W. (2008). A community change in the algal endosymbionts of a scleractinian coral following a natural bleaching event: Field evidence of acclimatization. Proceedings of the Royal Society B: Biological Sciences, 275(1641), 1359-1365. https://doi.org/10.1098/rspb.2008.0069

Jones, L. W. \& Kok, B. (1966). Photoinhibition of Chloroplast Reactions. I. Kinetics and Action Spectra. Physiology (Vol. 41).

Jones, R. J. \& Hoegh-Guldberg, O. (2001). Diurnal changes in the photochemical efficiency of the symbiotic dinoflagellates (Dinophyceae) of corals: Photoprotection, photoinactivation and the relationship to coral bleaching. Plant, Cell and Environment, 24(1), 89-99. https://doi.org/10.1046/j.1365-3040.2001.00648.x 
Jones, R. J., Hoegh-Guldberg, O., Larkum, A. W. D. \& Schreiber, U. (1998). Temperature-induced bleaching of corals begins with impairment of the $\mathrm{CO} 2$ fixation mechanism in zooxanthellae. Plant, Cell and Environment, 21(12), 1219-1230. https://doi.org/10.1046/j.13653040.1998.00345.x

Kaplan, A., Badger, M. R. \& Berry, J. A. (1980). Photosynthesis and the intracellular inorganic carbon pool in the bluegreen alga Anabaena variabilis: Response to external CO2 concentration. Planta, 149(3), 219-226.

Kemp, D. W., Thornhill, D. J., Rotjan, R. D., Iglesias-Prieto, R., Fitt, W. K. \& Schmidt, G. W. (2015). Spatially distinct and regionally endemic Symbiodinium assemblages in the threatened Caribbean reef-building coral Orbicella faveolata. Coral Reefs, 34(2), 535-547. https://doi.org/10.1007/s00338-015-1277-z

Keren, N., Berg, A., Van Kan, P. J. M., Levanon, H. \& Ohad, I. (1997). Mechanism of Photosystem II Photoinactivation and D1 Protein Degradation at Low Light: The Role of Back Electron Flow. Source (Vol. 94).

Klughammer, C. \& Schreiber, U. (2008). Complementary PS II quantum yields calculated from simple fluorescence parameters measured by PAM fluorometry and the Saturation Pulse method. PAM Application Notes, 1(1), 27-35. https://doi.org/citeulike-article-id:6352156

Komenda, J. \& Masojídek, J. (1998). The effect of Photosystem II inhibitors DCMU and BNT on the high-light induced D1 turnover in two cyanobacterial strains Synechocystis PCC 6803 and Synechococcus PCC 7942. Photosynthesis Research (Vol. 57).

Kramer, D. M., Johnson, G., Kiirats, O. \& Edwards, G. E. (2004). New fluorescence parameters for the determination of QA redox state and excitation energy fluxes. Photosynthesis Research, 79(2), 209-218. https://doi.org/10.1023/B:PRES.0000015391.99477.0d

Krieger, A. \& Weis, E. (1993). The role of calcium in the pH-dependent control of Photosystem II. Photosynthesis Research (Vol. 37).

Krueger, T. (2017). Concerning the cohabitation of animals and algae - an English translation of K. Brandt's 1881 presentation "Ueber das Zusammenleben von Thieren und Algen." Symbiosis, 71(3), 167-174. https://doi.org/10.1007/s13199-016-0439-2

Kulk, G., Poll, W. H. \& Buma, A. G. J. (2018). Photophysiology of nitrate limited phytoplankton communities in Kongsfjorden, Spitsbergen. Limnology and Oceanography, 63(6), 2606-2617. https://doi.org/10.1002/lno.10963

Kulk, G., van de Poll, W. H., Visser, R. J. W. \& Buma, A. G. J. (2013). Low nutrient availability reduces high-irradiance-induced viability loss in oceanic phytoplankton. Limnology and Oceanography, 58(5), 1747-1760. https://doi.org/10.4319/lo.2013.58.5.1747

Kurien, B. T. \& Scofield, R. H. (2009). Introduction to protein blotting. Methods in Molecular Biology (Clifton, N.J.), 536, 9-22. https://doi.org/10.1007/978-1-59745-542-8_3

LaJeunesse, T. C. (2001). Investigating the biodiversity, ecology, and phylogeny of endosymbiotic dinoflagellates in the genus Symbiodinium using the its region: In search of a "species" level marker. Journal of Phycology, 37(5), 866-880. https://doi.org/10.1046/j.15298817.2001.01031.x

LaJeunesse, T. C., Parkinson, J. E., Gabrielson, P. W., Jeong, H. J., Reimer, J. D., Voolstra, C. R. \& Santos, S. R. (2018). Systematic Revision of Symbiodiniaceae Highlights the Antiquity and Diversity of Coral Endosymbionts. Current Biology, 28(16), 2570-2580.e6. https://doi.org/10.1016/j.cub.2018.07.008

Lajeunesse, T. C. \& Trench, R. K. (2000). Biogeography of two species of Symbiodinium (Freudenthal) inhabiting the intertidal sea anemone Anthopleura elegantissima (Brandt). 
Biological Bulletin, 199(2), 126-134. https://doi.org/10.2307/1542872

Le Goff, C., Ganot, P., Zoccola, D., Caminiti-Segonds, N., Allemand, D. \& Tambutté, S. (2016). Carbonic anhydrases in cnidarians: Novel perspectives from the octocorallian corallium rubrum. PLoS ONE, 11(8), e0160368-e0160368. https://doi.org/10.1371/journal.pone.0160368

Lesser, M. P. (2019). Phylogenetic signature of light and thermal stress for the endosymbiotic dinoflagellates of corals (Family Symbiodiniaceae). Limnol. Oceanogr, 64, 1852-1863. https://doi.org/10.1002/lno.11155

Libby, P. S. \& Wheeler, P. A. (1997). Particulate and dissolved organic nitrogen in the central and eastern equatorial Pacific. Deep-Sea Research Part I: Oceanographic Research Papers, 44(2), 345-361. https://doi.org/10.1016/S0967-0637(96)00089-1

Liefer, J. D., Garg, A., Campbell, D. A., Irwin, A. J. \& Finkel, Z. V. (2018). Nitrogen starvation induces distinct photosynthetic responses and recovery dynamics in diatoms and prasinophytes. PLoS ONE, 13(4), 1-24. https://doi.org/10.1371/journal.pone.0195705

Liefer, J. D., Garg, A., Fyfe, M. H., Irwin, A. J., Benner, I., Brown, C. M., ... Finkel, Z. V. (2019). The macromolecular basis of phytoplankton C:N:P under nitrogen starvation. Frontiers in Microbiology, 10(MAR), 763. https://doi.org/10.3389/fmicb.2019.00763

Mansour, J. S., Pollock, F. J., Díaz-Almeyda, E., Iglesias-Prieto, R. \& Medina, M. (2018). Intra- and interspecific variation and phenotypic plasticity in thylakoid membrane properties across two Symbiodinium clades. Coral Reefs, 37(3), 841-850. https://doi.org/10.1007/s00338-018-1710-1

Manzello, D. P., Matz, M. V., Enochs, I. C., Valentino, L., Carlton, R. D., Kolodziej, G., ... Jankulak, M. (2019). Role of host genetics and heat-tolerant algal symbionts in sustaining populations of the endangered coral Orbicella faveolata in the Florida Keys with ocean warming. Global Change Biology, 25(3), 1016-1031. https://doi.org/10.1111/gcb.14545

Markham, J. W. \& Hagmeier, E. (1982). Observations on the effects of germanium dioxide on the growth of macro-algae and diatoms. Phycologia, 21(2), 125-130. https://doi.org/10.2216/i0031$8884-21-2-125.1$

Maruyama, S., Shoguchi, E., Satoh, N. \& Minagawa, J. (2015). Diversification of the light-harvesting complex gene family via intra- and intergenic duplications in the coral symbiotic alga Symbiodinium. PLoS ONE, 10(3). https://doi.org/10.1371/journal.pone.0119406

Matthews, J. L., Sproles, A. E., Oakley, C. A., Grossman, A. R., Weis, V. M. \& Davy, S. K. (2016). Menthol-induced bleaching rapidly and effectively provides experimental aposymbiotic sea anemones (Aiptasia sp.) for symbiosis investigations. https://doi.org/10.1242/jeb.128934

Mattoo, A. K., Pick, U., Hoffman-Falk, H. \& Edelman, M. (1981). The rapidly metabolized 32,000dalton polypeptide of the chloroplast is the'proteinaceous shield' regulating photosystem II electron transport and mediating diuron herbicide sensitivity. Proceedings of the National Academy of Sciences of the United States of America, 78(3 I), 1572-1576. https://doi.org/10.1073/pnas.78.3.1572

Mattoo, Autar K., Hoffman-Falk, H., Marder, J. B. \& Edelman, M. (1984). Regulation of protein metabolism: coupling of photosynthetic electron transport to in vivo degradation of the rapidly metabolized 32-kilodalton protein of the chloroplast membranes. Proceedings of the National Academy of Sciences of the United States of America, 20(1), 1380-1384. https://doi.org/10.1073/pnas.81.5.1380

Mattoo, Autar K, Pick, U., Hoffman-Falk, H. \& Edelman, M. (1981). The rapidly metabolized 32,000-dalton polypeptide of the chloroplast is the'proteinaceous shield' regulating photosystem II electron transport and mediating diuron herbicide sensitivity. Proceedings of the National Academy of Sciences of the United States of America, 78(3 I), 1572-1576. 
https://doi.org/10.1073/pnas.78.3.1572

McAuley, P. J. \& Cook, C. B. (1994). Effects of host feeding and dissolved ammonium on cell division and nitrogen status of zooxanthellae in the hydroid Myrionema amboinense. Marine Biology, 121(2), 343-348. https://doi.org/10.1007/BF00346743

McCabe Reynolds, J., Bruns, B. U., Fitt, W. K. \& Schmidt, G. W. (2008). Enhanced photoprotection pathways in symbiotic dinoflagellates of shallow-water corals and other cnidarians. Proceedings of the National Academy of Sciences of the United States of America, 105(36), 13674-13678. https://doi.org/10.1073/pnas.0805187105

McCloskey, L. R., Cove, T. G. \& Verde, E. A. (1996). Symbiont expulsion from the anemone Anthopleura elegantissima (Brandt) (Cnidaria; Anthozoa). Journal of Experimental Marine Biology and Ecology, 195(2), 173-186. https://doi.org/10.1016/0022-0981(95)00079-8

McGinley, M. P., Aschaffenburg, M. D., Pettay, D. T., Smith, R. T., LaJeunesse, T. C. \& Warner, M. E. (2012). Transcriptional Response of Two Core Photosystem Genes in Symbiodinium spp. Exposed to Thermal Stress. PLoS ONE, 7(12). https://doi.org/10.1371/journal.pone.0050439

Mehler, A. H. (1951). Studies on reactions of illuminated chloroplasts. I. Mechanism of the reduction of oxygen and other hill reagents. Archives of Biochemistry and Biophysics, 33(1), 65-77. https://doi.org/10.1016/0003-9861(51)90082-3

Mellis, A. (1999). Photosystem-II damage and repair cycle in chloroplasts: What modulates the rate of photodamage in vivo? Trends in Plant Science. https://doi.org/10.1016/S1360-1385(99)01387-4

Minagawa, J. (2013, December 17). Dynamic reorganization of photosynthetic supercomplexes during environmental acclimation of photosynthesis. Frontiers in Plant Science. Frontiers Research Foundation. https://doi.org/10.3389/fpls.2013.00513

Moejes, F. W., Matuszynska, A., Adhikari, K., Bassi, R., Cariti, F., Cogne, G., ... Ebenhöh, O. (2017). A systems-wide understanding of photosynthetic acclimation in algae and higher plants. Journal of Experimental Botany, 68(11), 2667-2681. https://doi.org/10.1093/jxb/erx137

Moritz, C. P. (2017). Tubulin or Not Tubulin: Heading Toward Total Protein Staining as Loading Control in Western Blots. PROTEOMICS, 17(20), 1600189. https://doi.org/10.1002/pmic.201600189

Morris, J. M. \& Fleming, G. R. (2018). Quantitative modeling of energy dissipation in Arabidopsis thaliana. Environmental and Experimental Botany, 154(March), 99-109. https://doi.org/10.1016/j.envexpbot.2018.03.021

Müller, M. L., Irkens-Kiesecker, U., Rubinstein, B. \& Taiz, L. (1996). On the mechanism of hyperacidification in lemon: Comparison of the vacuolar H+-ATPase activities of fruits and epicotyls. Journal of Biological Chemistry, 271(4), 1916-1924. https://doi.org/10.1074/jbc.271.4.1916

Muscatine, L. (1967). Glycerol Excretion by Symbiotic Algae from Corals and Tridacna and Its Control by the Host. Science, 156(3774), 516-519.

Muscatine, L., Falkowski, P. G., Dubinsky, Z., Cook, P. A. \& McCloskey, L. R. (1989). The effect of external nutrient resources on the population dynamics of zooxanthellae in a reef coral.

Proceedings of the Royal Society of London, B, 236(1284), 311-324. https://doi.org/10.1098/rspb.1989.0025

Muscatine, Leonard \& Cernichiari, E. (1969). Assimilation of photosynthetic products of Zooxanthellae by a reef coral. The Biological Bulletin, 137(3), 506-523. https://doi.org/10.2307/1540172

Nath, K., Jajoo, A., Poudyal, R. S., Timilsina, R., Park, Y. S., Aro, E. M., .. Lee, C. H. (2013). 
Towards a critical understanding of the photosystem II repair mechanism and its regulation during stress conditions. FEBS Letters, 587(21), 3372-3381.

https://doi.org/10.1016/j.febslet.2013.09.015

Nishiyama, Y., Allakhverdiev, S. I. \& Murata, N. (2006, July). A new paradigm for the action of reactive oxygen species in the photoinhibition of photosystem II. Biochimica et Biophysica Acta - Bioenergetics. https://doi.org/10.1016/j.bbabio.2006.05.013

Nishiyama, Y., Allakhverdiev, S. I., Yamamoto, H., Hayashi, H. \& Murata, N. (2004). Singlet oxygen inhibits the repair of photosystem II by suppressing the translation elongation of the D1 protein in Synechocystis sp. PCC 6803. Biochemistry, 43(35), 11321-11330. https://doi.org/10.1021/bi036178q

Nishiyama, Y., Yamamoto, H., Allakhverdiev, S. I., Inaba, M., Yokota, A. \& Murata, N. (2001). Oxidative stress inhibits the repair of photodamage to the photosynthetic machinery. EMBO Journal, 20(20), 5587-5594. https://doi.org/10.1093/emboj/20.20.5587

Nixon, P. J., Sarcina, M. \& Diner, B. A. (2006). The D1 and D2 Core Proteins. In Photosystem II (pp. 71-93). Springer-Verlag. https://doi.org/10.1007/1-4020-4254-x_5

Niyogi, K. K. \& Truong, T. B. (2013). Evolution of flexible non-photochemical quenching mechanisms that regulate light harvesting in oxygenic photosynthesis. Current Opinion in Plant Biology, 16, 307-314. https://doi.org/10.1016/j.pbi.2013.03.011

Ogren, W. L. (1984). Photorespiration: Pathways, regulation and modification. Annual Review of Plant Physiology, 35, 415-442.

Ohad, I., Kyle, D. J. \& Arntzen, C. J. (1984). Membrane protein damage and repair: removal and replacement of inactivated 32-kilodalton polypeptides in chloroplast membranes. The Journal of Cell Biology, 99(2), 481-485. https://doi.org/10.1083/jcb.99.2.481

Ohnishi, N., Allakhverdiev, S. I., Takahashi, S., Higashi, S., Watanabe, M., Nishiyama, Y. \& Murata, N. (2005). Two-step mechanism of photodamage to photosystem II: Step 1 occurs at the oxygenevolving complex and step 2 occurs at the photochemical reaction center. Biochemistry, 44(23), 8494-8499. https://doi.org/10.1021/bi047518q

Oulhen, N., Schulz, B. J. \& Carrier, T. J. (2016). English translation of Heinrich Anton de Bary's 1878 speech, 'Die Erscheinung der Symbiose' ('De la symbiose'). Symbiosis, 69(3), 131-139. https://doi.org/10.1007/s13199-016-0409-8

Papageorgiou, G. C. \& Govindjee. (2004). Chlorophyll a Fluorescence (Vol. 19). https://doi.org/10.1007/978-1-4020-3218-9

Papina, M., Meziane, T. \& Van Woesik, R. (2003). Symbiotic zooxanthellae provide the host-coral Montipora digitata with polyunsaturated fatty acids. Comparative Biochemistry and Physiology B Biochemistry and Molecular Biology, 135(3), 533-537. https://doi.org/10.1016/S10964959(03)00118-0

Park, A. Y., Anderson, J. M., Chow, W. S., Park, Y., Anderson, J. M. \& Chow, W. S. (1996). Photoinactivation of functional photosystem II and D1-prot synthesis in vivo are independent of the modulation of the photosynthetic apparatus by growth. Planta, 198(2), 300-309.

Park, Y.-I., Chow, W. S. \& Anderson, J. M. (1995). Light inactivation of functional photosystem II in leaves of peas grown in moderate light depends on photon exposure. Source: Planta (Vol. 196). Retrieved from https://www.jstor.org/stable/23383417?seq=1\&cid=pdfreference\#references_tab_contents

Parkinson, J. E. \& Baums, I. B. (2014). The extended phenotypes of marine symbioses: Ecological and evolutionary consequences of intraspecific genetic diversity in coral-algal associations. Frontiers in Microbiology, 5(AUG), 445. https://doi.org/10.3389/fmicb.2014.00445 
Peltier, G. \& Cournac, L. (2002). CHLORORESPIRATION. Annual Review of Plant Biology, 53(1), 523-550. https://doi.org/10.1146/annurev.arplant.53.100301.135242

Pernice, M., Meibom, A., Van Den Heuvel, A., Kopp, C., Domart-Coulon, I., Hoegh-Guldberg, O. \& Dove, S. (2012). A single-cell view of ammonium assimilation in coral-dinoflagellate symbiosis. ISME Journal, 6(7), 1314-1324. https://doi.org/10.1038/ismej.2011.196

Pillai-Kastoori, L., Schutz-Geschwender, A. R. \& Harford, J. A. (2020, March 15). A systematic approach to quantitative Western blot analysis. Analytical Biochemistry. Academic Press Inc. https://doi.org/10.1016/j.ab.2020.113608

Pochon, X., LaJeunesse, T. C. \& Pawlowski, J. (2004). Biogeographic partitioning and host specialization among foraminiferan dinoflagellate symbionts (Symbiodinium; Dinophyta). Marine Biology, 146(1), 17-27. https://doi.org/10.1007/s00227-004-1427-2

Pochon, X., Pawlowski, J., Zaninetti, L. \& Rowan, R. (2001). High genetic diversity and relative specificity among Symbiodinium-like endosymbiotic dinoflagellates in soritid foraminiferans. Marine Biology, 139(6), 1069-1078. https://doi.org/10.1007/s002270100674

Pochon, Xavier \& Gates, R. D. (2010). A new Symbiodinium clade (Dinophyceae) from soritid foraminifera in Hawai'i. Molecular Phylogenetics and Evolution, 56(1), 492-497. https://doi.org/10.1016/j.ympev.2010.03.040

Powles, S. B. (1984). Photoinhibition of Photosynthesis Induced by Visible Light. Annual Review of Plant Physiology, 35(1), 15-44. https://doi.org/10.1146/annurev.pp.35.060184.000311

Rädecker, N., Pogoreutz, C., Voolstra, C. R., Wiedenmann, J. \& Wild, C. (2015). Nitrogen cycling in corals: The key to understanding holobiont functioning? Trends in Microbiology, 23(8), 490497. https://doi.org/10.1016/j.tim.2015.03.008

Rahav, O., Dubinsky, Z., Achituv, Y. \& Falkowski, P. G. (1989). Ammonium metabolism in the zooxanthellate coral, stylophora pistillata. Proceedings of the Royal Society of London. B. Biological Sciences, 236(1284), 325-337. https://doi.org/10.1098/rspb.1989.0026

Roberts, J. M., Fixter, L. M. \& Davies, P. S. (2001). Ammonium metabolism in the symbiotic sea anemone Anemonia viridis. Hydrobiologia (Vol. 461).

Roberty, S., Fransolet, D., Cardol, P., Plumier, J. C. \& Franck, F. (2015). Imbalance between oxygen photoreduction and antioxidant capacities in Symbiodinium cells exposed to combined heat and high light stress. Coral Reefs, 34(4), 1063-1073. https://doi.org/10.1007/s00338-015-1328-5

Roberty, Stéphane, Bailleul, B., Berne, N., Franck, F. \& Cardol, P. (2014). PSI Mehler reaction is the main alternative photosynthetic electron pathway in Symbiodinium sp., symbiotic dinoflagellates of cnidarians. New Phytologist, 204(1), 81-91. https://doi.org/10.1111/nph.12903

Romero-Torres, M., Acosta, A., Palacio-Castro, A. M., Treml, E. A., Zapata, F. A., Paz-García, D. A. \& Porter, J. W. (2020). Coral reef resilience to thermal stress in the Eastern Tropical Pacific. Global Change Biology, 26(7), 3880-3890. https://doi.org/10.1111/gcb.15126

Rouzé, H., Lecellier, G. J., Saulnier, D., Planes, S., Gueguen, Y., Wirshing, H. H. \& BerteauxLecellier, V. (2017). An updated assessment of symbiodinium spp. that associate with common scleractinian corals from moorea (French Polynesia) reveals high diversity among background symbionts and a novel finding of clade B. PeerJ, 2017(1). https://doi.org/10.7717/peerj.2856

Rowan, R \& Powers, D. (1991a). A molecular genetic classification of zooxanthellae and the evolution of animal-algal symbioses. Science, 251(4999), 1348-1351.

Rowan, R \& Powers, D. (1991b). Molecular genetic identification of symbiotic dinoflagellates (zooxanthellae). Marine Ecology Progress Series, 71(1), 65-73.

https://doi.org/10.3354/meps071065 
Rowan, Rob \& Knowlton, N. (1995). Intraspecific diversity and ecological zonation in coral-algal symbiosis. Proceedings of the National Academy of Sciences of the United States of America, 92(7), 2850-2853. https://doi.org/10.1073/pnas.92.7.2850

Rowan, Rob, Knowlton, N., Baker, A. \& Jara, J. (1997). Landscape ecology of algal symbionts creates variation in episodes of coral bleaching. Nature, 388(6639), 265-269. https://doi.org/10.1038/40843

Ruban, A. V. (2016). Nonphotochemical chlorophyll fluorescence quenching: Mechanism and effectiveness in protecting plants from photodamage. Plant Physiology, 170(4), 1903-1916. https://doi.org/10.1104/pp.15.01935

Ruban, A. V., Johnson, M. P. \& Duffy, C. D. P. (2012). The photoprotective molecular switch in the photosystem II antenna. Biochimica et Biophysica Acta - Bioenergetics, 1817(1), 167-181. https://doi.org/10.1016/j.bbabio.2011.04.007

Sacharz, J., Giovagnetti, V., Ungerer, P., Mastroianni, G. \& Ruban, A. V. (2017). The xanthophyll cycle affects reversible interactions between PsbS and light-harvesting complex II to control non-photochemical quenching, 16225. https://doi.org/10.1038/nplants.2016.225

Sampayo, E. M., Dove, S. \& Lajeunesse, T. C. (2009). Cohesive molecular genetic data delineate species diversity in the dinoflagellate genus Symbiodinium. Molecular Ecology, 18(3), 500-519. https://doi.org/10.1111/j.1365-294X.2008.04037.x

Sanchez, F. G., Díaz, A. N. \& García, J. A. G. (1995). P-phenol derivatives as enhancers of the chemiluminescent luminol-horseradish peroxidase- $\mathrm{H} 2 \mathrm{O} 2$ reaction: substituent effects. Journal of Luminescence, 65(1), 33-39. https://doi.org/10.1016/0022-2313(95)00047-T

Schreiber, U. (2007). Pulse-Amplitude-Modulation (PAM) Fluorometry and Saturation Pulse Method: An Overview. In Chlorophyll a Fluorescence (pp. 279-319). Springer Netherlands. https://doi.org/10.1007/978-1-4020-3218-9_11

Schreiber, U., Klughammer, C. \& Kolbowski, J. (2012). Assessment of wavelength-dependent parameters of photosynthetic electron transport with a new type of multi-color PAM chlorophyll fluorometer. Photosynthesis Research, 113(1-3), 127-144. https://doi.org/10.1007/s11120-0129758-1

Shea, R. \& Chopin, T. (2007). Effects of germanium dioxide, an inhibitor of diatom growth, on the microscopic laboratory cultivation stage of the kelp, Laminaria saccharina. Journal of Applied Phycology, 19(1), 27-32. https://doi.org/10.1007/s10811-006-9107-x

Shikanai, T. (2014). Central role of cyclic electron transport around photosystem I in the regulation of photosynthesis. Current Opinion in Biotechnology, 26(Figure 1), 25-30. https://doi.org/10.1016/j.copbio.2013.08.012

Signore, M., Manganelli, V. \& Hodge, A. (2017). Antibody validation by Western blotting. Methods in Molecular Biology, 1606, 51-70. https://doi.org/10.1007/978-1-4939-6990-6_4

Silverstein, R. N., Correa, A. M. S. \& Baker, A. C. (2012). Specificity is rarely absolute in coral-algal symbiosis: implications for coral response to climate change, 279(1738), 2609-2618. https://doi.org/10.1098/rspb.20

Silverstein, R. N., Cunning, R. \& Baker, A. C. (2015). Change in algal symbiont communities after bleaching, not prior heat exposure, increases heat tolerance of reef corals. Global Change Biology, 21(1), 236-249. https://doi.org/10.1111/gcb.12706

Silverstein, R. N., Cunning, R. \& Baker, A. C. (2017). Tenacious D: Symbiodinium in clade D remain in reef corals at both high and low temperature extremes despite impairment. Journal of Experimental Biology, 220(7), 1192-1196. https://doi.org/10.1242/jeb.148239 
Smith, D. J., Suggett, D. J. \& Baker, N. R. (2005). Is photoinhibition of zooxanthellae the primary cause of thermal bleaching in corals? Global Change Biology, 11(September), 1-11. https://doi.org/10.1111/j.1365-2486.2004.00895.x

Smith, E. G., D'Angelo, C., Salih, A. \& Wiedenmann, J. (2013). Screening by coral green fluorescent protein (GFP)-like chromoproteins supports a role in photoprotection of zooxanthellae. Coral Reefs, 32(2), 463-474. https://doi.org/10.1007/s00338-012-0994-9

Stat, M. \& Gates, R. D. (2011). Clade D Symbiodinium in Scleractinian Corals: A "Nugget” of Hope, a Selfish Opportunist, an Ominous Sign, or All of the Above? Journal of Marine Biology, 2011. https://doi.org/10.1155/2011/730715

Suggett, D. J., Goyen, S., Evenhuis, C., Szabó, M., Pettay, D. T., Warner, M. E. \& Ralph, P. J. (2015a). Functional diversity of photobiological traits within the genus Symbiodinium appears to be governed by the interaction of cell size with cladal designation. New Phytologist, 208(2), 370-381. https://doi.org/10.1111/nph.13483

Suggett, D. J., Goyen, S., Evenhuis, C., Szabó, M., Pettay, D. T., Warner, M. E. \& Ralph, P. J. (2015b). Functional diversity of photobiological traits within the genus Symbiodinium appears to be governed by the interaction of cell size with cladal designation. New Phytologist, 208(2), 370-381. https://doi.org/10.1111/nph.13483

Suggett, D. J., Prasil, O. \& Borowitzka, M. A. (2010). Chlorophyll a Fluorescence In Aquatic Sciences - Methods and Applications.

Suggett, D. J., Warner, M. E., Smith, D. J., Davey, P., Hennige, S. \& Baker, N. R. (2008). Photosynthesis and production of hydrogen peroxide by Symbiodinium (Pyrrhophyta) phylotypes with different thermal tolerances. Journal of Phycology, 44(4), 948-956. https://doi.org/10.1111/j.1529-8817.2008.00537.x

Sully, S., Burkepile, D. E., Donovan, M. K., Hodgson, G. \& van Woesik, R. (2019). A global analysis of coral bleaching over the past two decades. Nature Communications, 10(1), 1-5. https://doi.org/10.1038/s41467-019-09238-2

Sutton, D. C. \& Hoegh-Guldberg, O. (1990). Host-Zooxanthella Interactions in Four Temperate Marine Invertebrate Symbioses: Assessment of Effect of Host Extracts on Symbionts. The Biological Bulletin, 178(2), 175-186. https://doi.org/10.2307/1541975

Swain, T. D., Chandler, J., Backman, V. \& Marcelino, L. (2017). Consensus thermotolerance ranking for 110 Symbiodinium phylotypes: an exemplar utilization of a novel iterative partial-rank aggregation tool with broad application potential. Functional Ecology, 31(1), 172-183. https://doi.org/10.1111/1365-2435.12694

Swain, T. D., Westneat, M. W., Backman, V. \& Marcelino, L. A. (2018). Phylogenetic analysis of symbiont transmission mechanisms reveal evolutionary patterns in thermotolerance and host specificity that enhance bleaching resistance among vertically transmitted Symbiodinium. European Journal of Phycology, 53(4), 443-459. https://doi.org/10.1080/09670262.2018.1466200

Takahashi, S. \& Murata, N. (2006). Glycerate-3-phosphate, produced by CO2 fixation in the Calvin cycle, is critical for the synthesis of the D1 protein of photosystem II. Biochimica et Biophysica Acta-Bioenergetics, 1757(3), 198-205. https://doi.org/10.1016/j.bbabio.2006.02.002

Takahashi, S. \& Murata, N. (2008). How do environmental stresses accelerate photoinhibition? Trends in Plant Science. https://doi.org/10.1016/j.tplants.2008.01.005

Takahashi, S., Nakamura, T., Sakamizu, M., Van Woesik, R. \& Yamasaki, H. (2004). Repair Machinery of Symbiotic Photosynthesis as the Primary Target of Heat Stress for Reef-Building Corals. Plant and Cell Physiology, 45(2), 251-255. https://doi.org/10.1093/pcp/pch028 
Taylor, D. L. (1969). The nutritional relationship of Anemonia sulcata (Pennant) and its dinoflagellate symbiont. Journal of Cell Science, 4(3), 751-762.

Taylor, S. C., Berkelman, T., Yadav, G. \& Hammond, M. (2013). A defined methodology for reliable quantification of western blot data. Molecular Biotechnology, 55(3), 217-226. https://doi.org/10.1007/s12033-013-9672-6

Taylor, S. C. \& Posch, A. (2014). The design of a quantitative western blot experiment. BioMed Research International. https://doi.org/10.1155/2014/361590

Thinesh, T., Meenatchi, R., Jose, A., Kiran, G. S. \& Selvin, J. (2019). Differential bleaching and recovery pattern of southeast Indian coral reef to 2016 global mass bleaching event: Occurrence of stress-tolerant symbiont Durusdinium (Clade D) in corals of Palk Bay.

https://doi.org/10.1016/j.marpolbul.2019.05.033

Thurber, R. L. V., Burkepile, D. E., Fuchs, C., Vega Thurber, R. L., Burkepile, D. E., Fuchs, C., ... Zaneveld, J. R. (2014). Chronic nutrient enrichment increases prevalence and severity of coral disease and bleaching. Global Change Biology, 544-554. https://doi.org/10.1111/gcb.12450

Titlyanov, E., Bil', K., Fomina, I., Titlyanova, T., Leletkin, V., Eden, N., ... Dubinsky, Z. (2000). Effects of dissolved ammonium addition and host feeding with Artemia salina on photoacclimation of the hermatypic coral Stylophora pistillata.

Tivey, T. R., Parkinson, J. E. \& Weis, V. M. (2020). Host and Symbiont Cell Cycle Coordination Is Mediated by Symbiotic State, Nutrition, and Partner Identity in a Model CnidarianDinoflagellate Symbiosis. MBio, 11(2). https://doi.org/10.1128/mBio.02626-19

Toller, W. W., Rowan, R. \& Knowlton, N. (2001). Zooxanthellae of the Montastraea annularis Species Complex: Patterns of Distribution of Four Taxa of Symbiodinium on Different Reefs and across Depths. Source: Biological Bulletin (Vol. 201). Retrieved from https://about.jstor.org/terms

Towbin, H., Staehelin, T. \& Gordon, J. (1979). Electrophoretic Transfer of Proteins from Polyacrylamide Gels to Nitrocellulose Sheets: Procedure and Some Applications (Vol. 76).

Tyystjärvi, E. (2008). Photoinhibition of Photosystem II and photodamage of the oxygen evolving manganese cluster. Coordination Chemistry Reviews, 252, 361-376. https://doi.org/10.1016/j.ccr.2007.08.021

Tyystjärvi, E., Ali-Yrkkö, K., Kettunen, R. \& Aro, E. M. (1992). Slow degradation of the D1 protein is related to the susceptibility of low-light-grown pumpkin plants to photoinhibition. Plant Physiology, 100(3), 1310-1317. https://doi.org/10.1104/pp.100.3.1310

Tyystjarvi, E. \& Aro, E.-M. (1996). The rate constant of photoinhibition, measured in lincomycintreated leaves, is directly proportional to light intensity (photosynthesis/photosystem II/DI protein/chlorophyll fluorescence). Plant Biology (Vol. 93). Retrieved from https://about.jstor.org/terms

UN Environment ISU ICRI \& Trucost. (2018). The Coral Reef Economy. International Sustainability Unit, 36. Retrieved from www.unenvironment.org

van Oevelen, D., Vermeij, M. J. A., Middelburg, J. J., de Goeij, J. M., Osinga, R., de Goeij, A. F. P. M. \& Admiraal, W. (2013). Surviving in a Marine Desert: The Sponge Loop Retains Resources Within Coral Reefs. Science, 342(6154), 108-110. https://doi.org/10.1126/science.1241981

van Oppen, M. (2001). In vitro establishment of symbiosis in Acropora millepora planulae. Coral Reefs, 20(3), 200. https://doi.org/10.1007/s003380100167

Vass, I. (2012). Molecular mechanisms of photodamage in the Photosystem II complex. Biochimica et Biophysica Acta - Bioenergetics. https://doi.org/10.1016/j.bbabio.2011.04.014 
Vass, I., Styring, S., Hundal, T., Koivuniemi, A., Aro, E.-M. \& Andersson, B. (1992). Reversible and Irreversible Intermediates during Photoinhibition of Photosystem II: Stable Reduced Q A Species Promote Chlorophyll Triplet Formation. Source (Vol. 89).

Wall, C. B., Kaluhiokalani, M., Popp, B. N., Donahue, M. J. \& Gates, R. D. (2020). Divergent symbiont communities determine the physiology and nutrition of a reef coral across a lightavailability gradient. ISME Journal, 2. https://doi.org/10.1038/s41396-019-0570-1

Wang \& Douglas. (1998). Nitrogen recycling or nitrogen conservation in an alga-invertebrate symbiosis? The Journal of Experimental Biology, 201 (Pt 16, 2445-53. Retrieved from http://www.ncbi.nlm.nih.gov/pubmed/9679106

Wangpraseurt, D., Holm, J. B., Larkum, A. W. D., Pernice, M., Ralph, P. J., Suggett, D. J. \& Kühl, M. (2017). In vivo Microscale Measurements of Light and Photosynthesis during Coral Bleaching: Evidence for the Optical Feedback Loop? Frontiers in Microbiology, 8(JAN), 59. https://doi.org/10.3389/fmicb.2017.00059

Ware, M. A., Belgio, E. \& Ruban, A. V. (2015). Photoprotective capacity of non-photochemical quenching in plants acclimated to different light intensities. Photosynthesis Research, 126(2-3), 261-274. https://doi.org/10.1007/s11120-015-0102-4

Warner, M. E., Fitt, W. K. \& Schmidt, G. W. (1999). Damage to Photosystem II in Symbiotic Dinoflagellates. A Determinant of Coral Bleaching. Source (Vol. 96).

Warner, M. E., Fittt, W. K. \& Schmidt, G. W. (1999). Damage to Photosystem II in Symbiotic Dinoflagellates . A Determinant of Coral Bleaching Author ( s ): Mark E . Warner, William K . Fitt and Gregory W. Schmidt Source: Proceedings of the National Academy of Sciences of the United States of America, Pu. Pnas, 96(14), 8007-8012.

Warner, M. E. \& Suggett, D. J. (2016). The Photobiology of Symbiodinium spp.: Linking Physiological Diversity to the Implications of Stress and Resilience. In S. Goffredo \& Z. Dubinsky (Eds.), The Cnidaria, Past, Present and Future The world of Medusa and her sisters (pp. 489-509). Springer International Publishing Switzerland. https://doi.org/10.1007/978-3319-31305-4_30

Weis, V. M. (1993). Effect of dissolved inorganic carbon concentration on the photosynthesis of the symbiotic sea anemone Aiptasia pulchella Carlgren: Role of carbonic anhydrase. Journal of Experimental Marine Biology and Ecology, 174(2), 209-225. https://doi.org/10.1016/00220981(93)90018-J

Weis, V. M. \& Reynolds, W. S. (1999). Carbonic anhydrase expression and synthesis in the sea anemone Anthopleura elegantissima are enhanced by the presence of dinoflagellate symbionts. Physiological and Biochemical Zoology, 72(3), 307-316. https://doi.org/10.1086/316674

Widder, E. A. (2010). Bioluminescence in the Ocean. Science, 704(5979), 704-708. Retrieved from http://www.sciencemag.org/cgi/doi/10.1126/science.1174269

Wooldridge, S. A. (2013). Breakdown of the coral-algae symbiosis: Towards formalising a linkage between warm-water bleaching thresholds and the growth rate of the intracellular zooxanthellae. Biogeosciences, 10(3), 1647-1658. https://doi.org/10.5194/bg-10-1647-2013

Xiang, T., Lehnert, E., Jinkerson, R. E., Clowez, S., Kim, R. G., DeNofrio, J. C., ... Grossman, A. R. (2020). Symbiont population control by host-symbiont metabolic interaction in Symbiodiniaceae-cnidarian associations. Nature Communications, 11(1), 1-9. https://doi.org/10.1038/s41467-019-13963-z

Yellowlees, D., Rees, T. A. V. \& Leggat, W. (2008). Metabolic interactions between algal symbionts and invertebrate hosts. Plant, Cell and Environment, 31(5), 679-694.

https://doi.org/10.1111/j.1365-3040.2008.01802.x 
Young, J. J. (2009). Detection and Quantification of Proteins on Immunoblots using Enhanced Chemiluminescence, 779-788. https://doi.org/10.1007/978-1-59745-198-7_77

Zavafer, A., Cheah, H., Hillier, W., Chow, W. S., Takahashi, S., Cheah, M. H., ... Takahashi, S. (2015). Photodamage to the oxygen evolving complex of photosystem II by visible light. Scientific Reports, 5, 1-9. https://doi.org/10.1038/srep16363 